University of Tennessee Health Science Center

UTHSC Digital Commons

$12-2013$

\title{
Development and Application of a BioRobotic Simulation of Stance Phase Gait to Study Foot and Ankle Kinematics
}

Kelly N. Salb

University of Tennessee Health Science Center

Follow this and additional works at: https://dc.uthsc.edu/dissertations

Part of the Equipment and Supplies Commons, and the Investigative Techniques Commons

\section{Recommended Citation}

Salb, Kelly N. , "Development and Application of a BioRobotic Simulation of Stance Phase Gait to Study Foot and Ankle Kinematics" (2013). Theses and Dissertations (ETD). Paper 223. http://dx.doi.org/ 10.21007/etd.cghs.2013.0272.

This Thesis is brought to you for free and open access by the College of Graduate Health Sciences at UTHSC Digital Commons. It has been accepted for inclusion in Theses and Dissertations (ETD) by an authorized administrator of UTHSC Digital Commons. For more information, please contact jwelch30@uthsc.edu. 


\title{
Development and Application of a BioRobotic Simulation of Stance Phase Gait to Study Foot and Ankle Kinematics
}

\begin{abstract}
Ankle instantaneous axis of rotation (IAR) measurements represent a more complete parameter for characterizing joint motion. However, few studies have implemented this measurement to study normal, injured, or pathological foot-ankle biomechanics. Additionally, while load is suggested to play a major role in ankle biomechanics, including influences on articular surfaces, bony motion, and formation of the arches, studies concerning the effects of joint loading are limited.

A novel testing protocol was developed to simulate in vivo mechanics of the foot-ankle complex during early stance phase gait in a human cadaveric model. Two studies were conducted. The first study was to assess the repeatability and accuracy of an existing robotic testing platform (RTP) and loading protocol using force measurements and IAR data from two cadaver specimens. A lower leg was mounted in a RTP with the tibia upright and foot flat on the baseplate. Axial tibia loads (ATLs) were controlled as a function of a vertical ground reaction force (vGRF) set at half body weight (356N) and a $50 \%$ vGRF (178N) Achilles tendon (AT) load. Two specimens were repetitively loaded over 10 degrees dorsiflexion and 20 degrees plantarflexion. Platform axes were controlled within $2 \mu \mathrm{m}$ and 0.008 degrees resulting in ATL measurements within $\pm 2 \mathrm{~N}$ of target conditions. Mean ATLs and IAR values were not significantly different between cycles of motion, but IAR values were between dorsiflexion and plantarflexion. A linear regression analysis showed no significant differences between slopes of plantarflexion paths.

The second study aimed to determine the effects of a passive (unloaded) and active Achilles and axial tibial loads on ankle mechanics using IAR data and translational and rotational data of the calcaneus, talus, and navicular from four cadaver specimens during stance phase gait. Specimens were mounted in the RTP with the tibia upright and foot flat on the baseplate. Passive loading applied a 5N ATL with no AT. Active ATLs were controlled as a function of a vGRF set at body weight (534N) and static ATs set at $25 \%$, $50 \%, 75 \%, 100 \%$ vGRF. Four specimens were repetitively loaded over 10 degrees dorsiflexion and 10 degrees plantarflexion. An optoelectric motion measuring system was used to track bony talus, calcaneus, and navicular translations and rotations. Kinematics in passive motion were predominantly governed by the shape of the mating articular surfaces. Once actively loaded, net joint loading had no surgically relevant effect on the kinematics data other than to suggest they were governed more by soft tissue structures.

The customized robotic platform and advanced testing protocol produced repeatable and accurate measurements of the IAR. Biomechanical properties of the foot and ankle were demonstrated, including the tibiotalar and soft tissue relationship on the axis of rotation and the effect of load on foot-ankle kinematics. The platform and protocol can be useful for assessing foot-ankle biomechanics under different loading scenarios and foot conditions, as well as studying the biomechanical effects of orthotics, footwear, and surgery or injury.
\end{abstract}

\section{Document Type}

Thesis

Degree Name

Master of Science (MS)

Program

Biomedical Engineering 


\section{Research Advisor}

Denis J. DiAngelo, Ph.D.

\section{Keywords}

Biomechanical Testing, Foot and Ankle, Gait Simulation, Kinematics, Robotics

\section{Subject Categories}

Analytical, Diagnostic and Therapeutic Techniques and Equipment | Equipment and Supplies | Investigative Techniques | Medicine and Health Sciences 


\title{
DEVELOPMENT AND APPLICATION OF A BIOROBOTIC SIMULATION OF STANCE PHASE GAIT TO STUDY FOOT AND ANKLE KINEMATICS
}

\author{
A Thesis \\ Presented for \\ The Graduate Studies Council \\ The University of Tennessee \\ Health Science Center
}

\begin{abstract}
In Partial Fulfillment
Of the Requirements for the Degree

Master of Science

In the Joint Graduate Program in Biomedical Engineering

From The University of Tennessee

and

The University of Memphis
\end{abstract}

By

Kelly N. Salb

December 2013 
Copyright (C) 2013 by Kelly N. Salb. All rights reserved. 


\section{DEDICATION}

"Find what you love and let it kill you." - Charles Bukowski 


\section{ACKNOWLEDGEMENTS}

Thank you to my research advisor, Dr. Denis DiAngelo, for your ongoing persistence and passion of knowledge that keeps your students motivated to do novel and rigorous research. Without that extra kick in the butt every now and then I think we would all still be current students. Also, thank you for those never-ending meetings that went on a multitude of tangents, leaving everyone confused. I have definitely gained insight into how to maintain focus and consider all aspects of an idea.

Of course none of this project could have ever been completed without the endless help of three lab members, each contributing to a significant aspect of this study. Tom Stewart, you kept me sane through learning NX6 and even more so when personal aspects threatened to halt my research. Dan Wido, you have taught me how to approach issues in the lab: how to test and prove an idea, think critically and thoroughly, and how to relax during endless hours of testing. You were by far my saving grace during the final months of testing. Hunter Smith, without you, no mechanical work would have ever been accomplished. Your help in the machine room and just simply talking about concepts were tremendous assets to my studies.

Finally, thank you to my sister and my father. Your care packages and comforting phone calls kept me motivated to finish and reduced the temptation to run away from Memphis. 


\begin{abstract}
Ankle instantaneous axis of rotation (IAR) measurements represent a more complete parameter for characterizing joint motion. However, few studies have implemented this measurement to study normal, injured, or pathological foot-ankle biomechanics. Additionally, while load is suggested to play a major role in ankle biomechanics, including influences on articular surfaces, bony motion, and formation of the arches, studies concerning the effects of joint loading are limited.

A novel testing protocol was developed to simulate in vivo mechanics of the footankle complex during early stance phase gait in a human cadaveric model. Two studies were conducted. The first study was to assess the repeatability and accuracy of an existing robotic testing platform (RTP) and loading protocol using force measurements and IAR data from two cadaver specimens. A lower leg was mounted in a RTP with the tibia upright and foot flat on the baseplate. Axial tibia loads (ATLs) were controlled as a function of a vertical ground reaction force (vGRF) set at half body weight (356N) and a $50 \%$ vGRF $(178 \mathrm{~N})$ Achilles tendon (AT) load. Two specimens were repetitively loaded over 10 degrees dorsiflexion and 20 degrees plantarflexion. Platform axes were controlled within $2 \mu \mathrm{m}$ and 0.008 degrees resulting in ATL measurements within $\pm 2 \mathrm{~N}$ of target conditions. Mean ATLs and IAR values were not significantly different between cycles of motion, but IAR values were between dorsiflexion and plantarflexion. A linear regression analysis showed no significant differences between slopes of plantarflexion paths.
\end{abstract}

The second study aimed to determine the effects of a passive (unloaded) and active Achilles and axial tibial loads on ankle mechanics using IAR data and translational and rotational data of the calcaneus, talus, and navicular from four cadaver specimens during stance phase gait. Specimens were mounted in the RTP with the tibia upright and foot flat on the baseplate. Passive loading applied a 5N ATL with no AT. Active ATLs were controlled as a function of a vGRF set at body weight $(534 \mathrm{~N})$ and static ATs set at $25 \%, 50 \%, 75 \%, 100 \%$ vGRF. Four specimens were repetitively loaded over 10 degrees dorsiflexion and 10 degrees plantarflexion. An optoelectric motion measuring system was used to track bony talus, calcaneus, and navicular translations and rotations. Kinematics in passive motion were predominantly governed by the shape of the mating articular surfaces. Once actively loaded, net joint loading had no surgically relevant effect on the kinematics data other than to suggest they were governed more by soft tissue structures.

The customized robotic platform and advanced testing protocol produced repeatable and accurate measurements of the IAR. Biomechanical properties of the foot and ankle were demonstrated, including the tibiotalar and soft tissue relationship on the axis of rotation and the effect of load on foot-ankle kinematics. The platform and protocol can be useful for assessing foot-ankle biomechanics under different loading scenarios and foot conditions, as well as studying the biomechanical effects of orthotics, footwear, and surgery or injury. 


\section{TABLE OF CONTENTS}

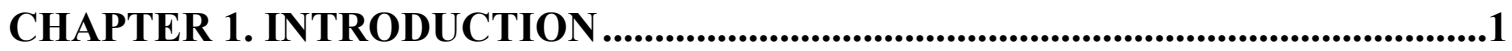

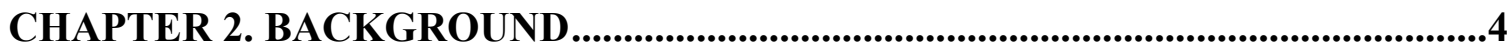

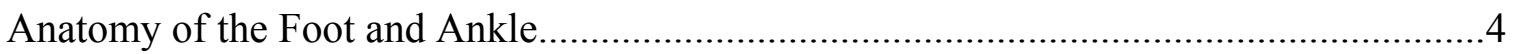

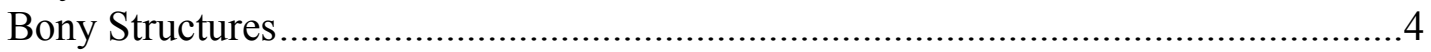

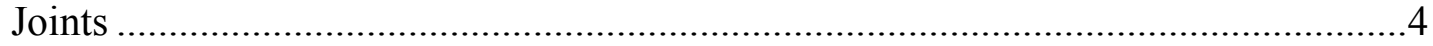

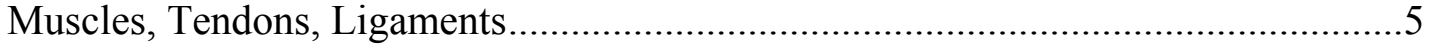

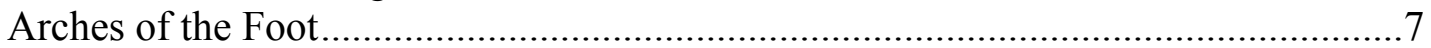

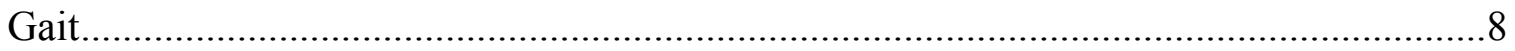

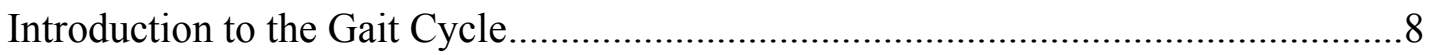

Range of Motion, Kinematics, and Kinetics ………….............................................

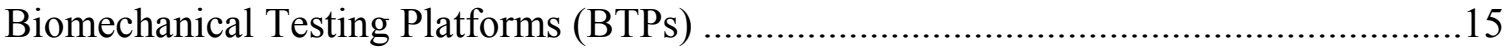

\section{CHAPTER 3. A NOVEL KINEMATIC BASED PROTOCOL TO STUDY}

FOOT AND ANKLE BIOMECHANICS ...........................................................19

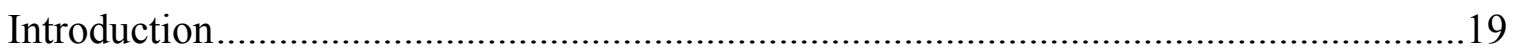

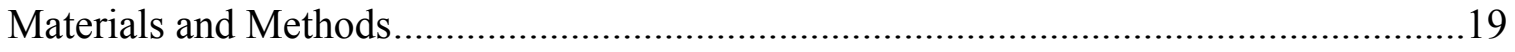

Tissue Preparation.........................................................................................19

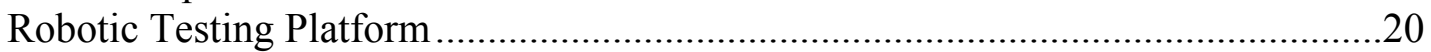

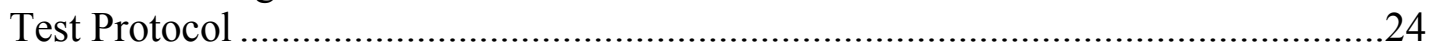

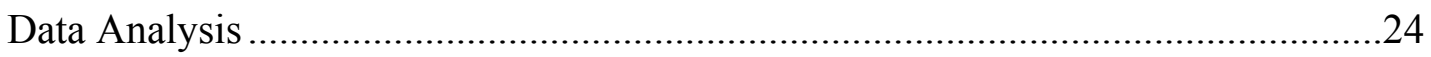

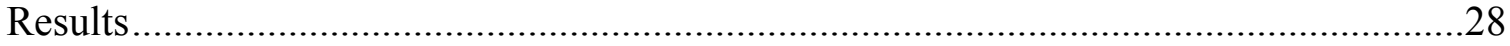

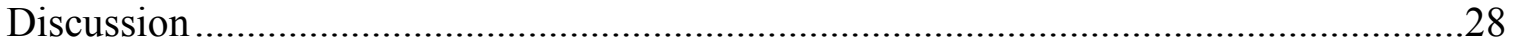

CHAPTER 4. EFFECTS OF ACHILLES LOADING ON THE

KINEMATIC PROPERTIES OF THE FOOT-ANKLE COMPLEX

DURING STANCE PHASE GAIT ..................................................................................33

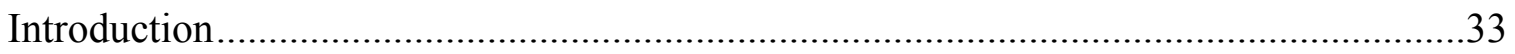

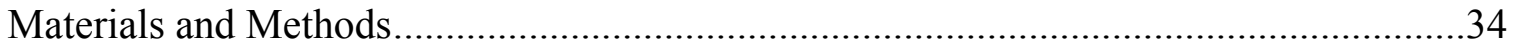

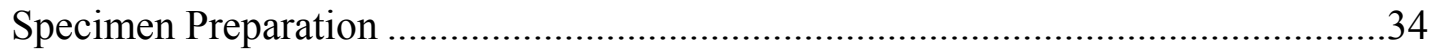

Robotic Testing Platform and Protocol....................................................................

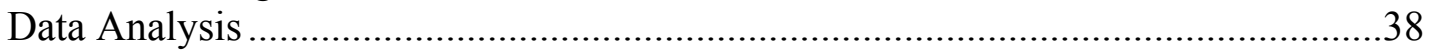

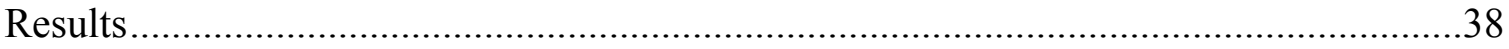

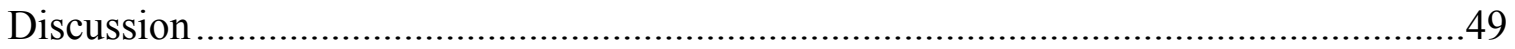

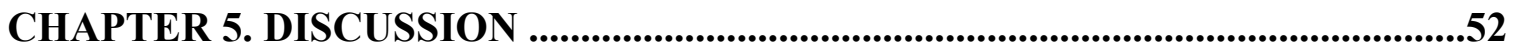

CHAPTER 6. CONCLUSION ...............................................................................................55

LIST OF REFERENCES ...................................................................................56

APPENDIX A. RADIOGRAPHS AND TRANSFORMATIONS .............................61 
APPENDIX B. MOTION MEASURING SYSTEM TRANSFORMATIONS........65

APPENDIX C. BONE TRANSLATIONS AND ROTATIONS................................66

APPENDIX D. RAW DATA

VITA 


\section{LIST OF TABLES}

Table 3-1. Average Axial Tibia Loading Conditions for Specimen between Cycles of Motion

Table 3-2. Mean IAR Values for Specimen 1 .29

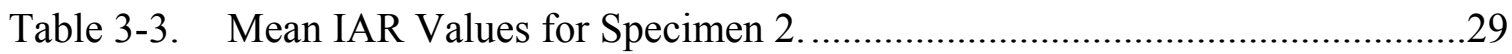

Table 4-1. Mean IAR Values between Loading Conditions for Specimen 1.................43

Table 4-2. Mean IAR Values between Loading Conditions for Specimen 2.................43

Table 4-3. Mean IAR Values between Loading Conditions for Specimen 3.................44

Table 4-4. Mean IAR Values between Loading Conditions for Specimen 4..................44

Table 4-5. Specimen 1 Translations and Rotations.......................................................45

Table 4-6. Specimen 2 Translations and Rotations..........................................................46

Table 4-7. Specimen 3 Translations and Rotations........................................................47

Table 4-8. Specimen 4 Translations and Rotations...........................................................48

Table A-1. Transformation Values for Program Input and Generic Axis Offsets ..........63

Table A-2. Talus Measurements and Ratio Comparison to Generic Model ....................64

Table C-1. Mean Translations for All Specimens.........................................................67

Table D-1. Forces (N) during Motion. .......................................................................

Table D-2. Validation Study IAR Values (mm) (Chapter 3)..........................................72

Table D-3. Kinematic Study IAR Values (mm) for Specimen 1 ...................................75

Table D-4. Kinematic Study IAR Values (mm) for Specimen 2 ...................................77

Table D-5. Kinematic Study IAR Values (mm) for Specimen 3 .................................79

Table D-6. Kinematic Study IAR Values $(\mathrm{mm})$ for Specimen 4...................................81 


\section{LIST OF FIGURES}

Figure 2-1. Lateral View of the Bony Structures in the Foot and Subdivisions...............4

Figure 2-2. Muscles and Tendons of the Foot and Ankle..........................................6

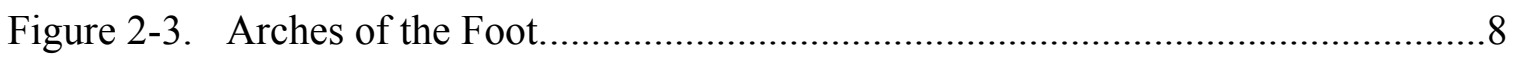

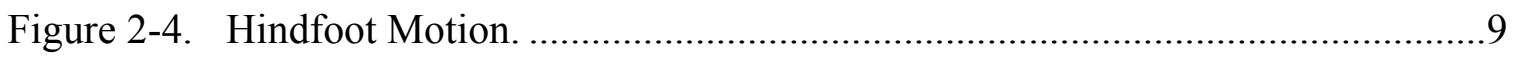

Figure 2-5. Realeaux's Graphical Method for Calculating the IAR..............................12

Figure 2-6. Rolling and Sliding Effect on IAR................................................... 12

Figure 2-7. In vitro Talocalcaneal Kinematic Data. ................................................13

Figure 2-8. EMG Activity for Six Muscles during Walking.....................................14

Figure 2-9. Free Body Diagram of Force Vectors and Moment Arms..........................16

Figure 2-10. BTPs That Investigate a Single Instance of Gait. ....................................17

Figure 2-11. Gait Simulators Engineered to Study Foot and Ankle Biomechanics. .........18

Figure 3-1. A Prepared below the Knee Lower Extremity Specimen. .........................21

Figure 3-2. Diagram of 4-DOF Robotic System Configured for the Ankle Study.........22

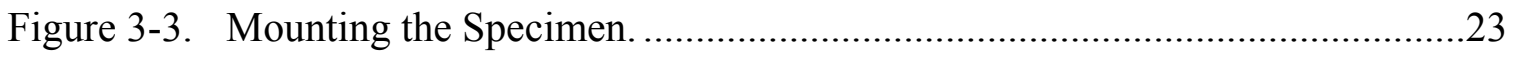

Figure 3-4. Force Analysis of the Foot and Ankle Used to Set the Parameters of

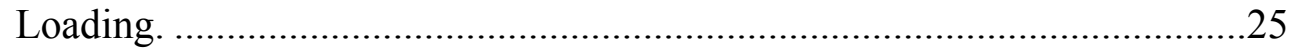

Figure 3-5. Modified "Pure Moment” Program. ........................................................26

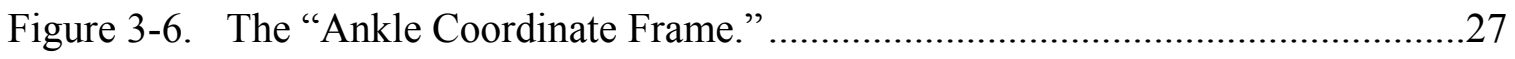

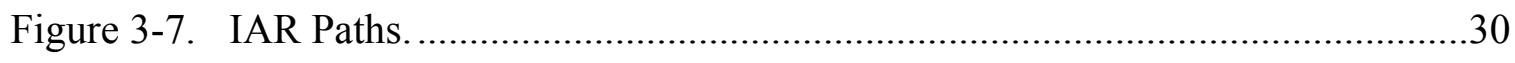

Figure 4-1. Diagram of 4-DOF Robotic System Configured for the Ankle Study..........35

Figure 4-2. Modified "Pure Moment” Program. ..........................................................

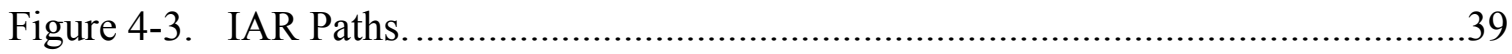

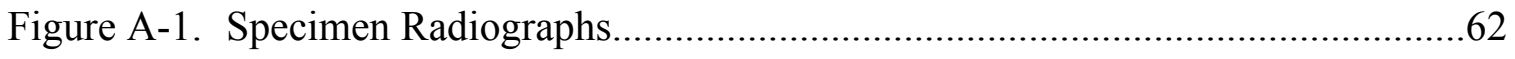

Figure A-2. Example of Extended Tool Tip and Generic Axis Transformations. ..........63 
Figure A-3. Talus Measurements.

Figure C-1. Bone Translations (Means and Standard Deviations) for All Specimens.....68

Figure C-2. Bone Rotations (Means and Standard Deviations) for All Specimens........69 


\section{LIST OF ABBREVIATIONS}

\begin{tabular}{ll} 
ARA & Ankle Rotational Axis \\
AT & Achilles Tendon \\
BTP & Biomechanical Testing Platform \\
BW & Body Weight \\
COR & Center of Rotation \\
EDL & Extensor Digitorum Longus \\
EHL & Extensor Hallucis Longus \\
EMG & Electromyographic \\
ETT & Extended Tool Tip \\
F $_{\text {a }}$ & Achilles Force \\
FC & Foot Contact \\
FF & Flat Foot \\
FHL & Flexor Hallucis Longus \\
F $_{t}$ & Tibia Force \\
GA & Gastrocnemius \\
GRF & Ground Reaction Force \\
HR & Heel Rise \\
Hz & Hertz \\
IAR & Instantaneous Axis of Rotation \\
Lbs & Pounds \\
LR & Loading Response \\
MMS & Motion Measuring System \\
N & Newtons \\
Nm & Newton Meters \\
NTT & Null Tool Tip \\
pARA & Prescribed Ankle Rotational Axis \\
PB & Peroneus Brevis \\
PL & Peroneus Longus \\
PO & Push Off \\
ROM & Range of Motion \\
RTP & Robotic Testing Platform \\
SO & Soleus \\
STJ & Subtalar Joint \\
TA & Tibialis Anterior \\
TAR & Total Ankle Replacement \\
TCJ & Talocrural Joint \\
TNJ & Talonavicular Joint \\
TO & Toe Off \\
TP & Tibialis Posterior \\
TS & Triceps Surae \\
vGRF & Vertical Ground Reaction Force \\
Wf & Weight of the Foot \\
& \\
\hline
\end{tabular}




\section{CHAPTER 1. INTRODUCTION}

Due to its inherent complexity and physical significance in human activities, the ankle complex is of great importance to researchers of degenerative diseases, injury prevention, and rehabilitation [1-4]. Each year, two million Americans visit the doctor with severe ankle pain due to arthritis, fractures, or sprains [5]. Approximately 25,000 patients undergo ankle fusions (arthrodesis) and roughly 4,400 undergo total ankle replacement (TAR) surgery (arthroplasty) to repair the joint each year [5]. The efficacy of TAR compared with that of ankle fusion continues to be one of the most debated topics in foot and ankle surgery [6-9]. Historically, ankle arthrodesis has been considered the "gold standard" for treatment of end-stage osteoarthritis with high patient satisfaction rates as a result of improved stability and reduced pain [6-9]. However, fusion severely inhibits ankle mobility, impacting gait mechanics and the adjacent joints, in turn increasing the risk of advanced adjacent joint degeneration [6-9]. Arthroplasty aims to imitate and restore physiologic function by regaining ankle mobility through two- or three-component tibio-talar implants, but has experienced high failure rates in the past [69]. While the evolution of TAR designs have improved in reducing failure rates, neither method perfectly mimics a physiologically healthy human ankle ultimately as a result of poor understanding of the joint mechanics [10-11].

Shoe inserts, or orthotics, and high arch support footwear are commonly used to treat fallen arches, foot pain, and plantar fasciitis, an inflammation of the plantar fascia, by limiting the stress on surrounding soft tissue structures $[2-4,12,13]$. However, debate between shoe and orthotic design involving minimal to considerable arch support has arisen among orthopaedists, podiatrists, and researchers over the past decade due to the limited knowledge of the biomechanical effects on the foot and ankle. Minimal support footwear lacking cushioning, arch support, and built up heels (Vibram FiveFingers, New Balance Minimus, Nike Free Run, etc.) have been becoming increasingly popular due to the more natural design, returning the foot to a more physiologic state [12]. Shoes with support were designed in the 1970's for safe and comfortable running [12]. Prior, running footwear was predominantly described as a "running flat." It is suggested that the foot accommodates the extra support by changing from a forefoot or midfoot strike pattern to a heel strike gait pattern $[2,3,12]$. While it is suggested that minimal support shoes may be the most natural design for healthy ambulation, limited knowledge is available on the biomechanical effects of orthotics and footwear on the healthy and pathologic foot.

In vitro studies [14-29], computational modeling [30-34], and in vivo testing [12, 35-48] have offered valuable insight into the mechanics of the ankle complex to aid in identification of injury, foot abnormality, surgical correction, and implant design by measuring forces and bony motion; however, each technique possesses some inherent limitations. In vivo studies involving X-ray photogrammetic [43], magnetic resonance imaging (MRI) [35,45,47,48], skin mounted retro-reflective markers [41,42,44,46], and videofluoroscopy [49] techniques are typically avoided. The amount of information they are able to acquire is limited due to the invasive surgeries required to obtain data 
$[44,47,48,50]$. Bone motion studies that use skin mounted retro-reflective markers suffer from inaccuracies due to skin-motion artifacts [50]. Additionally, in vivo data lack accurate and repeatable loading scenarios due to variability of responses in subjects $[35,50]$. Computational modeling is able to accurately and repeatably predict loading conditions, but is limited by access of validation data for both the healthy and altered (injured, pathological, or diseased) states, as well as an inability to include the role of soft tissue structures $[31,50]$. In contrast, in vitro cadaver studies are able to complement these techniques to produce clinically relevant data. However, orthopaedic research involving physiologic in vitro forces to bones, tendons, and ligaments during joint loading has often been limited by the biomechanical testing platforms (BTPs) [14-29].

To date very few BTPs are capable of applying physiologic joint loading conditions to the foot-ankle complex due to decreased in vivo loads or applied loads estimated by a single specimen $[14,15,17-20]$. Some studies only address loading conditions at a single instance of gait $[17,24,25,35]$. Of the platforms able to simulate walking gait, a forced kinematic profile is applied and data regarding the instantaneous axis of rotation (IAR) is not reported [14-23,25-29]. The ankle IAR is a more complete parameter for characterizing joint motion, where shifts in the IAR could help define injury type and/or the impact of injury on foot ankle mechanics, as well as the effects of surgical procedures and implant and orthotic design. Sammarco et al. confirmed that the center of rotation (COR) of a normal ankle does not remain constant with motion and that it lies within the cross-sectional area of the talus in the sagittal plane [30]. Additional studies suggested a fixed axis of rotation with articular congruence is an incorrect kinematic model for the ankle joint [11,31]. This concept was furthered by formulating the ankle joint behaves like a two-dimensional four-bar linkage during passive motion with an IAR about the sagittal plane [31-34]. This moving center of rotation was explained by suggesting the articular surfaces roll and slide upon each other during motion [11,31-34,36] and as axial load increases, contact between articular surfaces plays an increasingly greater role in the mechanics of the ankle and axis of rotation [38,51]. Load plays a major role in ankle biomechanics including influences on the articular surfaces, bony motion, and formation of the arches [36,51]. Arch formation is key in forming a rigid structure to transfer loads from the tibia to the ground. Activated primarily by the Achilles tendon (AT), it is suggested that once the arch is formed, little motion occurs between the calcaneus, navicular, and talus throughout stance phase $[2,14,20,25,36]$. This suggests most of the motion in the foot and ankle remains between the articular surfaces of the tibia and talus. However, studies concerning the effects of joint loading are limited. And still, many approximate a single point of rotation [16,35], assume the ankle joint behaves as a simple hinge joint [24], or define the COR using overly simplified techniques such as tracking landmarks over large angles radiographically $[35,38]$. Thus, there is demand for a more physiologic and accurate testing platform to evaluate foot and ankle mechanics that would enable accurate measurement and calculation of the IAR.

A novel testing protocol was developed to evaluate foot and ankle mechanics that used a multi-axis programmable robotic testing platform (RTP) to build on the traditional "pure moment" joint loading method by adding an Achilles load and an axial force 
through the tibia. The objectives of the current work were to: (1) Design a RTP and loading protocol that supported in vitro cadaveric testing of the foot and ankle complex during the early stance phase of gait, (2) Assess the repeatability and accuracy of the loading protocol using force measurements and IAR data from two cadaver specimens, and (3) Study the effects of a passive (unloaded) and active Achilles load and axial tibia load on ankle mechanics using IAR data and translational and rotational data of the calcaneus, talus, and navicular from four cadaver specimens during stance phase gait. 


\section{CHAPTER 2. BACKGROUND}

\section{Anatomy of the Foot and Ankle}

The anatomy of the human foot is extremely complex in that is consists of 26 bones, 33 joints, and more than one hundred muscles, tendons, and ligaments all working together to maintain the physiologic function of the foot.

\section{Bony Structures}

The bones of the foot can be subdivided into three sections: the hindfoot, midfoot, and forefoot (Figure 2-1) [52]. The hindfoot is comprised of the talus and calcaneus. The talus has the primary function of articulating with the tibia and fibula and transferring weight from the body to the calcaneus, midfoot, and forefoot. The midfoot consists of the cuboid, navicular, and three cuneiforms. And the forefoot is composed of five metatarsals, five proximal phalanges, four middle phalanges, and five distal phalanges. Of greatest interest to this study were the bones and articulations of the hindfoot since they are directly involved in the transfer of weight from the body to the ground. Therefore, the kinematics of these bones are used to define the cadaveric model of this protocol and thus the anatomy and relationships of the tibia, fibula, talus, calcaneus, and navicular are described in greater detail below.

\section{Joints}

The ankle joint, made up of the tibia, fibula, calcaneus, and talus, commonly referred to as the ankle complex, is involved in the transfer of weight from the body to

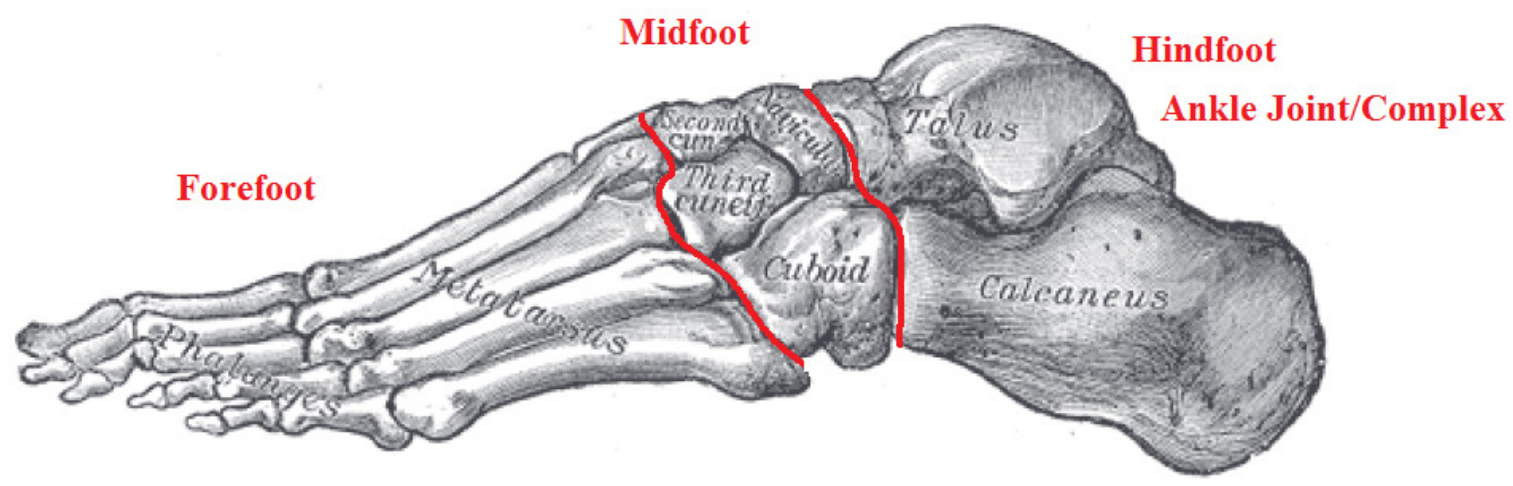

Figure 2-1. Lateral View of the Bony Structures in the Foot and Subdivisions. Source: Modified with permission. Gray, H. and Lewis, W.H. (1918). Anatomy of the human body. (pp.268). Philadelphia: Lea \& Febiger. 
the foot and furthermore, to the ground during gait [1-4,36-38,51]. It is made up of three articulations: the subtalar joint (STJ), the talocrural joint (TCJ), and the tibiofibular syndesmosis. The talar dome articulates with the tibial plafond, the smooth concave surface of the distal end of the tibia and the medial malleolus. The distal fibula, or lateral malleolus, and distal tibia form a mortise, in which the talus articulates to form the TCJ. Considerable variation in the shape of TCJ has been observed among subjects and is suspected to alter the biomechanics of gait in individuals [51]. The distal tibia and fibula articulate with each other to form the distal tibiofibular syndesmosis. The talocalcaneal, or STJ, is comprised of the anterior and posterior joints of the talus and calcaneus. Additionally, the transmission of forces through to the distal end of the foot is of great interest and therefore, the joint between the distal convex surface of the head of the talus and the proximal concave surface of the navicular, or talonavicular joint (TNJ), is considered.

Degeneration of the articulating cartilage of the TCJ is a condition called osteoarthritis [10]. Very common among middle age and older patients, treatment may involve ankle arthroplasty or arthrodesis, in which a surgeon will try to repair the joint. While arthrodesis fuses the tibia, fibula, and talus to remove the surface of the joint, arthroplasty regains the motion of the joint by replacing it with implants that mimic the physiologic function [5-10]. The efficacy of these surgical treatments remain a debated topic among orthopaedic surgeons.

\section{Muscles, Tendons, Ligaments}

The muscles acting on the foot can be classified into extrinsic and intrinsic muscles. Extrinsic muscles originate on the anterior or posterior aspect of the lower leg and have long tendons that cross the ankle and insert on bones in the foot [52]. On the other hand, intrinsic muscles originate on the dorsal or plantar aspects of the foot and are responsible for movement of the toes and supporting the arches of the foot [52].

Within the anterior extrinsic muscles are the extensors and peroneal groups. There are three major extensors: the tibialis anterior (TA), extensor digitorum longus (EDL), and extensor hallucis longus (EHL) (Figure 2-2) [52]. The TA originates on the anteriolateral surface of the tibia, descends over the front of the ankle, and inserts into the first cuneiform and metatarsal. The TA is the major dorsiflexor of the ankle and assists with foot inversion. The EDL originates on the lateral condyle of the tibia and anterior surface of the fibula, crosses over the front of the ankle, and inserts into the middle and distal phalanges. It functions to extend the distal phalanges and dorsiflex the ankle. The EHL attaches to the anterior surface of the fibula and the dorsal center of the distal phalanx of the big toe. It serves to extend the big toe and also aids with ankle dorsiflexion and inversion. In the peroneal group are the peroneus longus (PL), attached proximally to the head of the fibula and distally to the medial cuneiform and first metatarsal, and peroneus brevis (PB), originating at the lateral side of the fibula and attaching to the tuberosity on the lateral side of the fifth metatarsal (Figure 2-2). These are the main evertors of the foot, and also assist in plantarflexion of the ankle. 


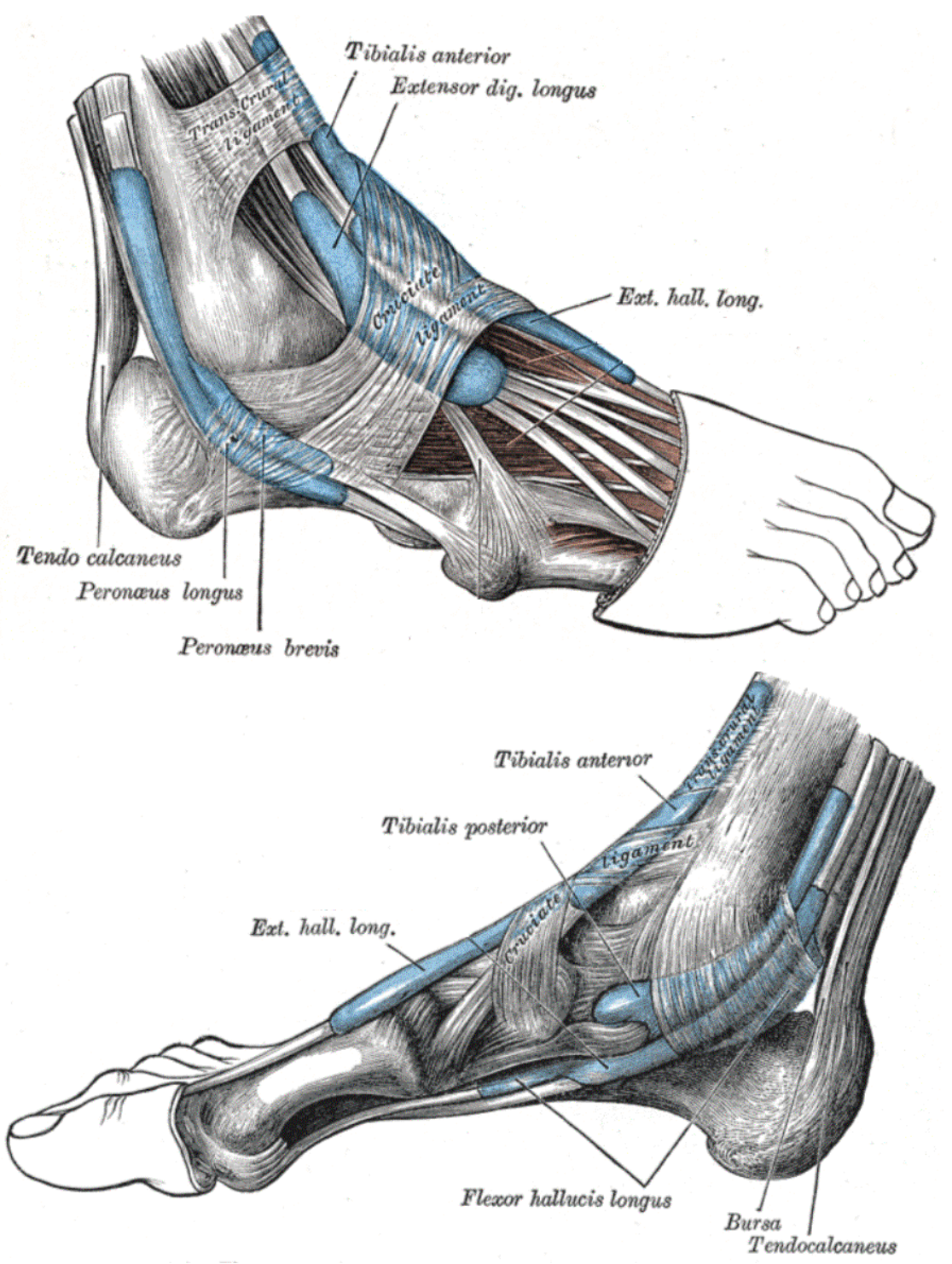

Figure 2-2. Muscles and Tendons of the Foot and Ankle.

Bursa is the plantaris. Source: Reprinted with permission. . Gray, H. and Lewis, W.H. (1918). Anatomy of the human body. (pp.268). Philadelphia: Lea \& Febiger. 
Within the posterior extrinsic muscles are triceps surae (TS), plantaris, tibialis posterior (TP), and flexor hallucis longus (FHL) (Figure 2-2) [52]. The triceps surae consists of the soleus (SO), originating on the posterior aspect of the tibia and fibula, and gastrocnemius (GA), originating on the posterior aspect of the femur. The plantaris originates at the inferior lateral ridge of the femur. The tendons of these muscles merge into the Achilles tendon (AT) that inserts on the calcaneus. Together, they serve as the main plantarflexors of the ankle. The TP originates on the posterior aspects of the tibia and fibula and descends posterior to the medial malleolus and terminates by dividing into three portions. The plantar portion inserts into the bases of the second, third and fourth metatarsals, the second and third cuneiforms and the cuboid. The main portion inserts into the tuberosity of the navicular and the plantar surface of the first cuneiform. The final portion inserts into the calcaneus. The TP is the main invertor of the foot, and aids the GA and SO with plantarflexion. The FHL arises from the distal two-thirds of the posterior surface of the fibula, descends on the medial side of the ankle, continues along the sole of the foot and is inserted into the base of the last phalanx of the great toe. It serves as the flexor of the big toe and assists with foot plantarflexion.

The ankle is supported by two groups of intrinsic ligaments: medial and lateral. Medially, the deltoid ligament binds the medial malleolus to the talus, calcaneus, and navicular. The lateral ligaments, two talofibular ligaments (anterior and posterior) and a calcaneofibular ligament, bind the lateral malleolus to the talus and calcaneus. Holding the tibiofibular syndesmosis are the distal tibiofibular ligaments (anterior and posterior). Together, these ligaments maintain the stability of the mortise and ankle joint [52].

\section{Arches of the Foot}

The human foot has two longitudinal arches and a transverse arch maintained by the interlocking shapes of the foot bones, ligaments, and muscles during activity (Figure 2-3) [52]. The slight mobility of these arches when weight is applied to and removed from the foot makes walking and running more economical in terms of energy [26]. The medial longitudinal arch curves above the ground. This arch is made up of the calcaneus, talus, navicular, three cuneiforms, and the first three medial metatarsals, with the talus serving as the "keystone" that holds the arch together. In contrast, the lateral longitudinal arch is very low. It redistributes part of the weight to the calcaneus, cuboid and the fourth and fifth metatarsals, with the cuboid as the "keystone." The two longitudinal arches serve as pillars for the transverse arch which runs obliquely across the tarsometatarsal joints, or the joints between the cuneiforms, cuboids, and the metatarsals. The medial longitudinal arch is supported by the TP, TA, AT and FHL. The lateral longitudinal arch is supported by the PB and PL, and the transverse arch is supported by the PL [2$4,11,26,36]$. The longitudinal arch is key in maintaining strength and rigidity of the foot during weight bearing. Excessive strain on the tendons and ligaments of the feet can result in fallen arches or flat feet [2-4,26,52]. 


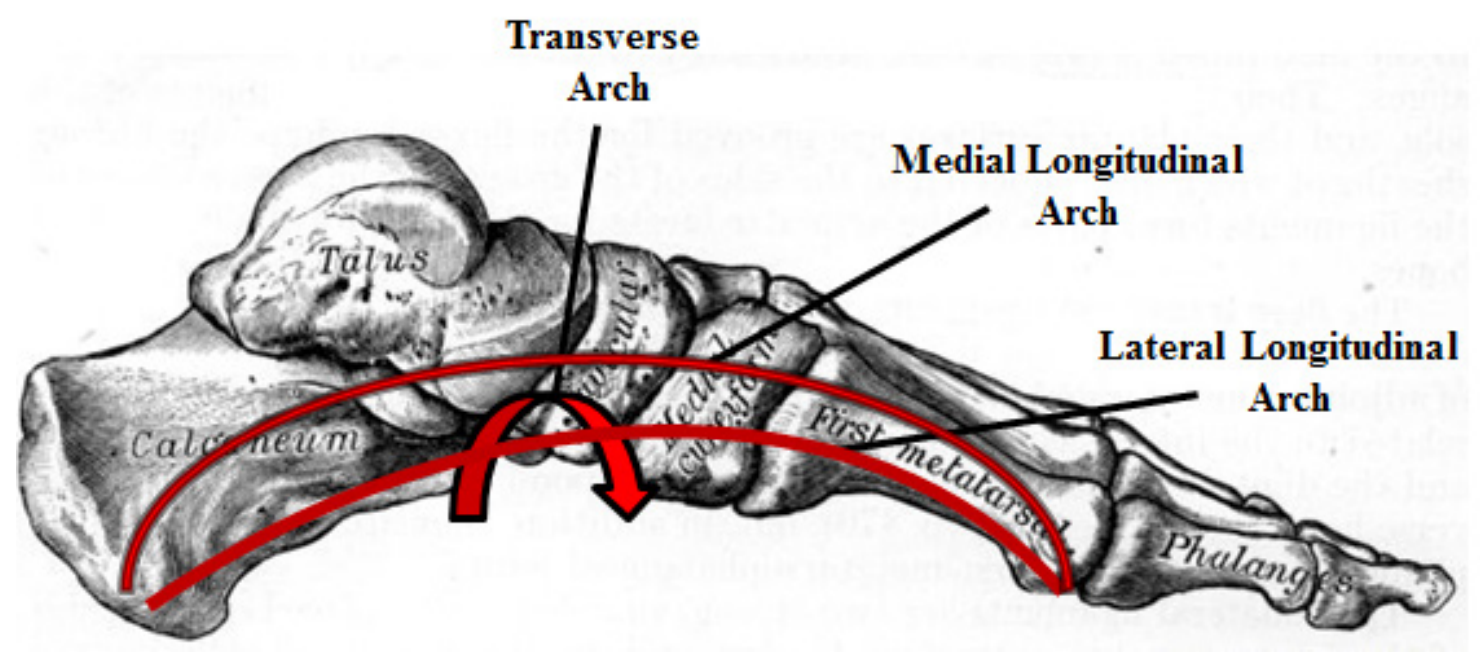

Figure 2-3. Arches of the Foot.

Source: Modified with permission: Gray, H. and Lewis, W.H. (1918). Anatomy of the human body. Philadelphia: Lea \& Febiger. p. 268.

\section{Gait}

\section{Introduction to the Gait Cycle}

Human locomotion is described by the gait cycle, characterized by the kinetics and kinematics of the lower extremities. Clinically, gait is divided into two distinct phases: stance phase, which comprises approximately 60 percent of the cycle, and swing phase, which accounts for the remaining 40 percent of gait [1-4,36,37]. The stance phase occurs when a portion or all of the foot is in contact with the ground. It is commonly described using six subdivisions: foot contact (FC), loading response (LR), flat foot (FF), heel rise (HR), push off (PO), and toe off (TO) [37]. This study aimed to investigate the early stance phase of gait with the foot flat on the ground and therefore, the swing phase was not addressed.

\section{Range of Motion, Kinematics, and Kinetics}

Kinematics of foot and ankle gait patterns aim to solely describe the motion of structures. There exists extensive data regarding the motion of the bony structures through gait by utilizing imaging techniques such as optoelectric motion tracking studies (MTS) in vitro and in vivo, as well as MRI and X-Ray measurements.

Hindfoot motion is often defined as occurring in in three cardinal planes: sagittal plane motion (plantarflexion-dorsiflexion), frontal plane motion (inversion-eversion), and transverse plane motion (abduction-adduction) (Figure 2-4) [4]. 


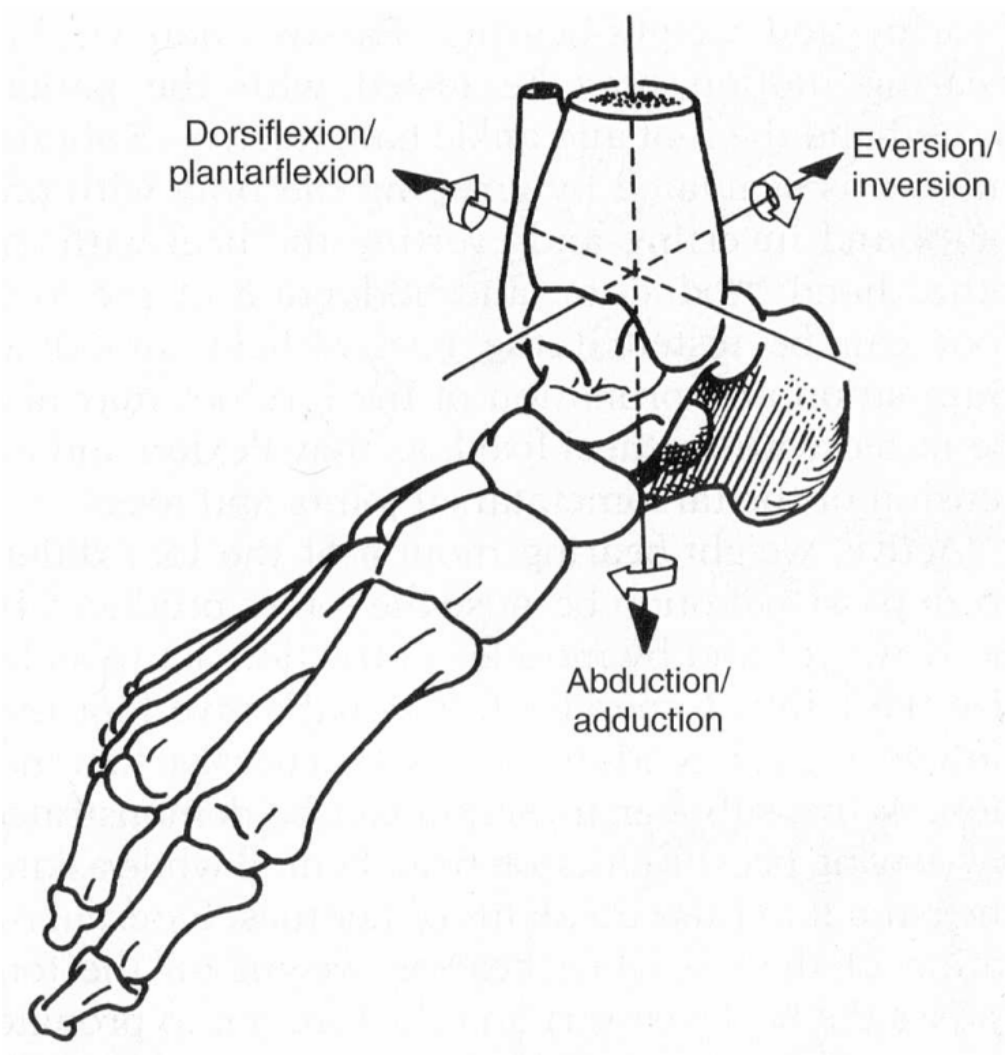

Figure 2-4. Hindfoot Motion.

Rotations in the three cardinal planes: sagittal plane motion (plantarflexion-dorsiflexion), frontal plane motion (inversion-eversion), and transverse plane motion (abductionadduction). Source: Reprinted with permission. Nordin, M. and V.H. Frankel, Basic Biomechanics of the Musculoskeletal System. 3rd ed. 2001, Philadelphia: Lippincott Williams \& Wilkins. p. 226. 
During FC ( $0 \%$ gait), body weight is transferred rapidly from one foot to the other as the foot comes to contact with the ground. In a normal healthy gait pattern, the heel is the first to come into contact with the foot slightly supinated (2-3 degrees) in a dorsiflexed state to prevent slapping of the foot [39]. During the loading response (0$10 \%$ gait) [37], the foot comes in full contact with the floor, and BW is fully transferred to the stance limb. The TA is used to lower the foot immediately after FC and acts to invert the foot when BW is completely on the heel [39]. The EDL and EHL also function as dorsiflexors to lower the foot, while the PL plays a major role in stabilizing the foot during this weight acceptance. At FC, the entire lower extremity including the tibia is rotated medially approximately 5 degrees. During LR, the medial rotation of the tibia causes a transfer of the acting pronatory moment from the ankle mortise to the talus and subsequently cause the subtalar joint to evert, and in turn the foot pronates (3-10 degrees) and becomes more flexible to absorb shock and adapt to load bearing [39]. The ankle then becomes plantarflexed as the forefoot comes into contact with the ground in FF, such that the weight is distributed between the heel and forefoot. At FF the lower limb rotates laterally, causing the foot to supinate, increasing stability along the longitudinal arches of the foot and the transverse tarsal joint [39]. Approximately 3-10 degrees of supination should occur until HR. The ankle then dorsiflexes, transferring weight from the hindfoot to the forefoot in preparation for HR (30\%) [37]. Weight distribution then moves entirely to the forefoot in PO, where the PL acts a plantarflexor to propel the body forward. During TO, all of the weight is transferred to the contralateral foot and the foot enters the swing phase. The GA and SO are active throughout stance phase, consistently rising in magnitude until reaching peak activity just before PO [37].

The TCJ is considered to have an axis of rotation with one degree of freedom in the sagittal plane during normal walking in dorsiflexion/plantarflexion [50]. Typical ranges of motion (ROM) values vary between walking and running, as well as between young healthy patients and elderly or injured patients. In dorsiflexion, typical values range from 5 to 20 degrees [39,51]. In plantarflexion, values range from 7 to 55 degrees [39,51]. Winters reported a maximum ROM of 9.6 degrees in dorsiflexion and 19.9 degrees in plantarflexion during walking in healthy adults [37]. However, functional ROMs have been shown to be approximately 5 degrees in dorsiflexion and 10 degrees in plantarflexion [51].

Sammarco et al. confirmed that the COR of a normal ankle does not remain constant with motion and that it lies within the cross-sectional area of the talus as viewed in the sagittal plane [30]. Additional studies suggested a fixed axis of rotation with articular congruence may be an incorrect kinematic model for the ankle joint [11]. This concept was furthered by formulating the ankle joint behaves like a two-dimensional four-bar linkage during passive motion and thus possesses an IAR about the sagittal plane [31-34]. The IAR measurement defines the point about which a rigid body undergoes rotation at a given instant in time. Therefore, IAR data provide additional parameters for characterizing tibiotalar joint motion, where measurements are a direct representation of the effects of joint articular geometry and soft tissue structures. The moving center of rotation was explained by suggesting the articular surfaces roll as well as slide upon each other during motion [11,31-34,36,51]. Realeaux developed a mathematical method to 
calculate the coordinates of the IAR $\left(\mathrm{X}_{\mathrm{cr}}, \mathrm{Y}_{\mathrm{cr}}\right)$ of a rigid body graphically by determining the intersection of the bisecting lines of the velocity vectors $\left(\bar{U}, \overline{U^{\prime}}\right)$ of two physiological landmarks (A \& B) through rotation $(\phi)$ (Figure 2-5)[53]. Using this concept, the effect of rolling and sliding can be shown using a simple ankle joint model (Figure 2-6). During rolling, contact is made on equal intervals of the articular surface, producing an upward axis of rotation on the joint articular surface. Sliding, with no influence of rolling would produce an infinitely downward IAR (bisectors run parallel to each other). Thus, the combination of rolling and sliding on the articular surface would produce an IAR within the talus that is dependent on the contribution of joint interaction.

As axial load increases, contact between articular surfaces plays an increasingly greater role in the mechanics of the ankle and COR [51]. While passive kinematics of the ankle complex are governed solely by the sliding of articular surfaces without any tissue deformation, active kinematics are governed more by soft tissue structures $[31,33]$. Studies have also shown that the four main intrinsic ligaments of the ankle joint are slack during walking and only taut at maximal ROM [31,51]. It thus becomes questionable whether the ligaments guide or limit joint motion for normal functional activities such as walking. Quantifying the IAR of the ankle joint during gait may have the potential to advance the understanding of the biomechanical properties of the foot and ankle, including arch formation and effects of orthotics and footwear.

The calcaneus and talus are believed to form a rigid, unified structure during weight bearing, such that no translation between the two bones exists throughout rotation of the foot (Figure 2-7)[1,14,20,36,44,50,46,50]. However, some studies have suggested there may be slight translations between the two structures due to kinetic properties and contact surface variability of the tibia on the talus [51]. Complex geometries of the mortise and trochlea of the talus greatly influence these load characteristics. The TCJ has shown considerable variation in shape between specimens. This may contribute to variations in functional ROM as well as maximal ROM. The geometry of the articulating surfaces primarily determines the mechanics of the TCJ. Data regarding contact area have been shown to vary from 1.5 to $9.4 \mathrm{~cm}^{2}$ depending on load and ankle position [51]. Goto et al. have shown if contact is maintained through rotation of the tibia, the TCJ and STJ function as joints with one degree of freedom of motion [40]. When pushed towards the maximal ROM, such as in anterior drawer tests and stress tests, congruent contact is not maintained, forcing a deviation from normal kinematics [51].

Kinetics study the forces that cause movement and are based on in vivo electromyographic (EMG) studies of the foot and ankle muscles during walking, as well as force plate studies of the ground reaction forces (GRFs). EMG activity for six muscles during walking is shown in Figure 2-8. Only activity in stance phase is discussed.

The TA, EDL, and PL act as stabilizers and dorsiflexors of the foot primarily during FC and PO [37]. However, only the flat foot portion of the stance phase of gait is incorporated in this model and therefore, these muscles are not considered. The major contributors of force to the ankle joint are the muscles that converge to the AT [37]. At $\mathrm{FC}$ the force is minimal, progressively getting larger until peak values are reached at PO. 


\section{Realeaux (1875) Graphical Method}

$\mathrm{t}_{1}$

$\mathrm{t}_{2}$

$t_{3}$

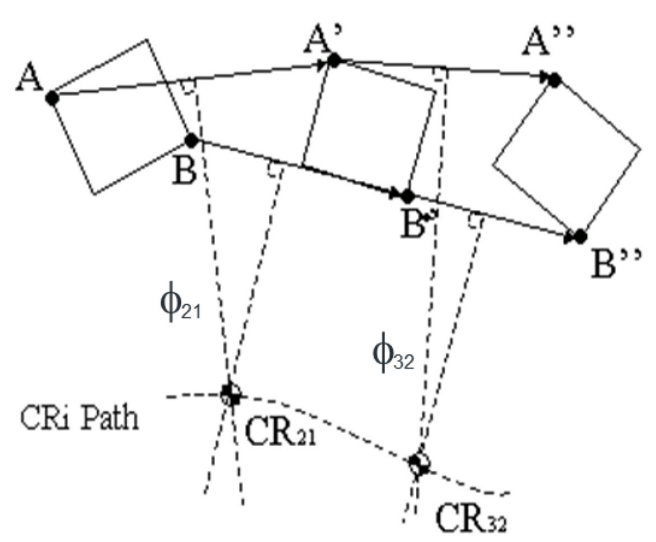

$\overline{\mathbf{u}}=\overline{\mathbf{A}}-\overline{\mathbf{B}}$, and $\overline{\mathbf{u}},=\overline{\mathbf{A}},-\overline{\mathbf{B}}$,

$\Phi=\cos ^{-1}\left(\frac{u \cdot u^{\prime}}{|u|\left|u^{\prime}\right|}\right)$

$X c r=\frac{1}{2}\left(x_{1}+x_{2}\right)+\frac{\left(y_{1}-y_{2}\right) \sin \phi}{2 *(1-\cos \phi)}$

$Y c r=\frac{1}{2}\left(y_{1}+y_{2}\right)-\frac{\left(x_{1}-x_{2}\right) \sin \phi}{2 *(1-\cos \phi)}$

Figure 2-5. Realeaux's Graphical Method for Calculating the IAR.

Two landmarks on a rigid body were tracked throughout rotation. Intersections of the perpendicular bisectors of the velocity vectors of the landmarks denote the IAR (CR).

The angles between the bisectors were determined graphically and used with the positions of the landmarks to calculate the IAR. Reprinted with permission: Crisco III, J.J., Chen, X., Panjabi, M.M., Wolfe, S.W. (1994). Optimal marker placement for calculating instantaneous center of rotation. J Biomech. 27(9), 1183-1187.

Pure Rolling

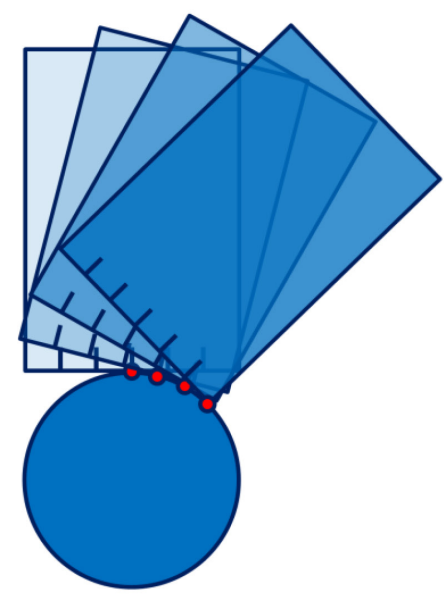

Pure Sliding

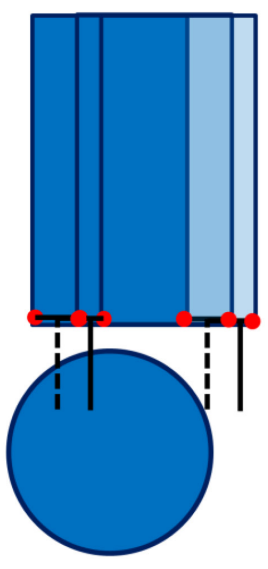

Rolling and Sliding

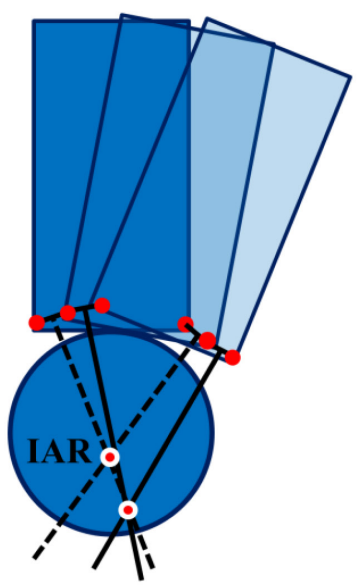

Figure 2-6. Rolling and Sliding Effect on IAR.

Simple model of the tibia and talus articular interaction. Rolling produces and IAR on the articular surface, sliding produces an infinitely downward IAR. 

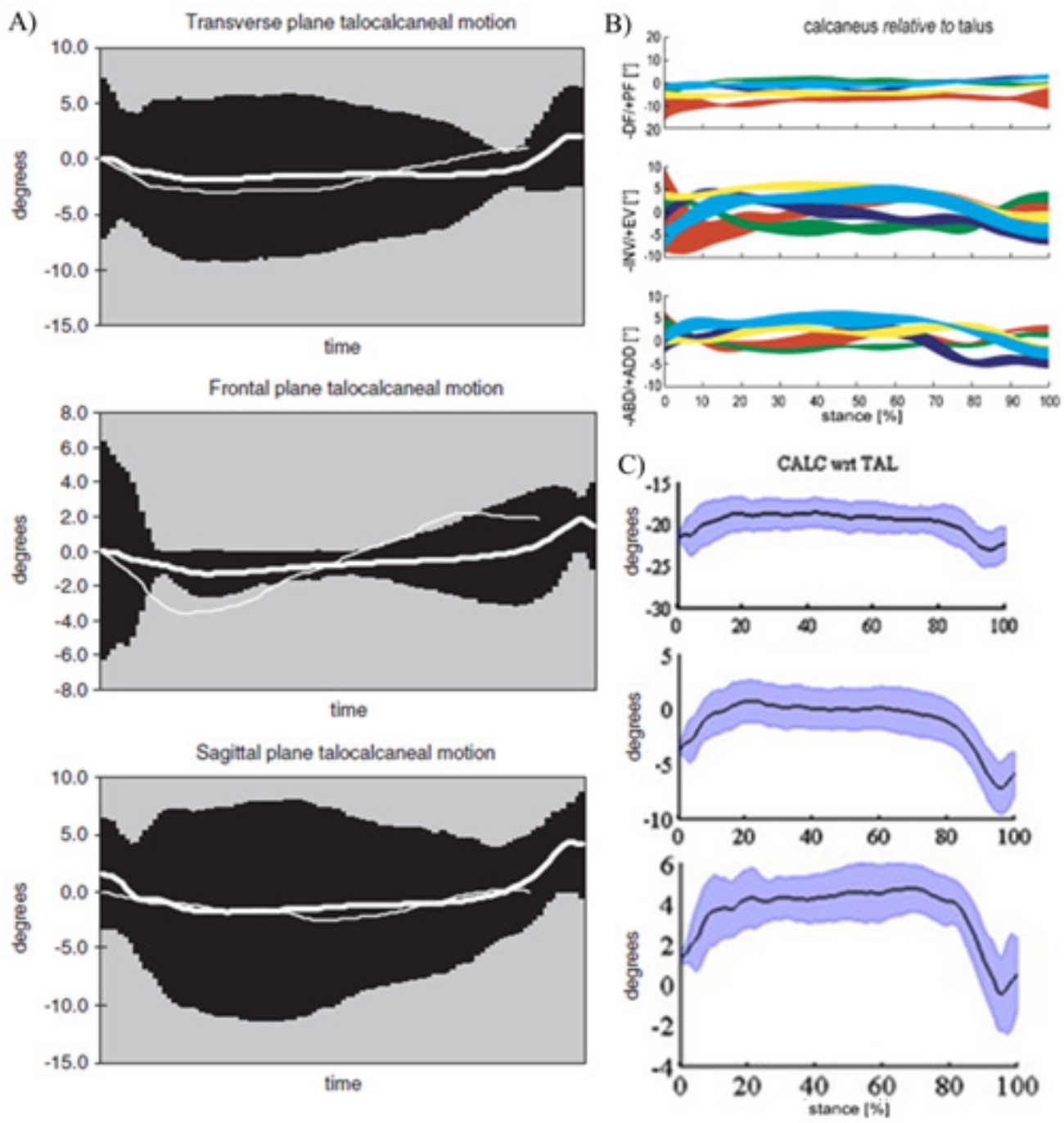

Figure 2-7. In vitro Talocalcaneal Kinematic Data.

Relative motion of the talus to the calcaneus through stance phase gait (0-100\%). A) Comparison of cadaveric kinematic data $(100 \%$ stance phase) $(n=13)$ (thin white line) to in vivo bone pin analyses (thick white line) $(n=3)$ with $95 \%$ confidence intervals. Positive angles denote plantarflexion, inversion, and adduction of the distal segment relative to the proximal. Source: Reprinted with permission. Nester, C.J., Liu, A.M., Ward, E., Howard, D., Cocheba, J., Derrick, T., Patterson, P. (2007). In vitro study of foot kinematics using a dynamic walking cadaver model. J. Biomech. 40(2), 1927-1937. B) Rearfoot kinematics for each specimen during stance phase $(0-100 \%)(n=5)$. Positive values denote plantarflexion, eversion, and adduction. Source: Reprinted with permission. Lundgren, P., Nester, C., Liu, A., Arndt, A., Jones, R., Stacoff, A., Lundberg, A. 2008. Invasive in vivo measurement of rear-, mid-and forefoot motion during walking. Gait \& Posture 28(1), 93-100. C) Relative calcaneus angular motion with respect to the talus $(\mathrm{n}=6)$. Positive values denote dorsiflexion, eversion, and abduction. Source: Reprinted with permission. Whittaker, E.C., Aubin, P.M., Ledoux, W.R. 2011. Foot bone kinematics as measured in a cadaver robotic gait simulator. Gait \& Posture 33, 645-650. 

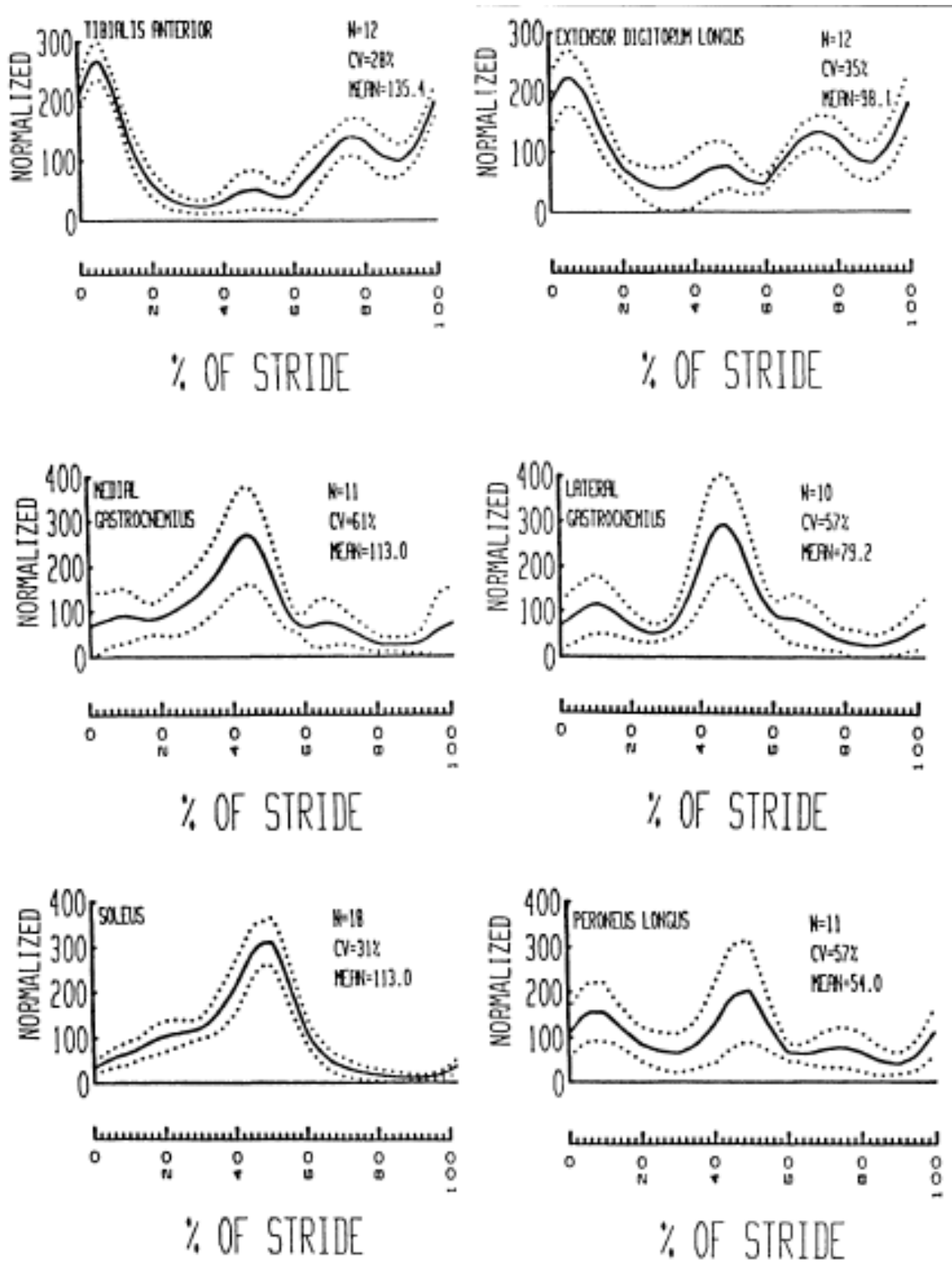

Figure 2-8. EMG Activity for Six Muscles during Walking.

Mean EMG (solid line) and standard deviations (dotted lines) are shown for varying sample sized. Values normalized to each subject's mean EMG. Source: Reprinted with permission. Winter, D.A., Yack, H.J., 1987. EMG profiles during normal human walking: Stride to stride and inter subject variability. Electroencaphalogr Clin Neurophysiol. 67: 402-411.

Typical values at $30 \%$ gait reach body weight (BW) with peak values at PO reaching $250 \%$ BW $[37,39,51]$. 
GRFs are commonly measured using force platforms in gait laboratories. GRFs show magnitude and direction of forces applied to the foot and ankle structures during locomotion. Peak values typically range from 1.1 to 1.3 times $\mathrm{BW}$, with average stance phase values around BW [39].

A rigid body analysis can be used to describe the dynamic loading characteristics of the ankle as a function of an AT force $\left(\mathrm{F}_{\mathrm{a}}\right)$ and vertical GRF (vGRF) (Figure 2-9) [54]. Assuming static equilibrium, the maximum vGRF is achieved in the neutral orientation at 0 degrees such that as a force (BW and weight of the foot $\left(\mathrm{W}_{\mathrm{f}}\right)$ ) is transmitted to the ground, the joint contact forces approximately equal the sum of the $\mathrm{vGRF}$ and $\mathrm{F}_{\mathrm{a}}$. With these forces are associated moment arms defined by the distance of the applied force to the joint center. Physiologically, as rotation occurs, the GRF moment arm (b) will move from the hindfoot in plantarflexion to the forefoot in dorsiflexion, which is accommodated by increased AT loads [51].

\section{Biomechanical Testing Platforms (BTPs)}

Several BTPs have been engineered to simulate loading of the ankle. However, very few BTPs offer a physiologic loading environment $[15,17,18,19]$ : either using reduced in vivo loads or neglecting tendon load incorporation. Some only investigate one instance of gait (Figure 2-10) [24,27,35,38]. Others apply loads or force a kinematic profile estimated by a single specimen (Figure 2-11) [15,17,20,21]. Some models assume the ankle complex behaves like a hinge joint with a single axis of rotation, whereas other studies suggested a fixed axis of rotation with articular congruence is an incorrect kinematic model for ankle joint motion $[11,30,31,36]$. To date, few studies have been conducted that address an instantaneous axis of rotation (IAR) analysis of the ankle joint $[23,35,38]$. These measurements are limited by the accuracy of the methodology. In these cases, radiologic measurements were made to determine the IAR. Additionally, measurements were made over large angle intervals reducing to the accuracy to as much as several millimeters. 


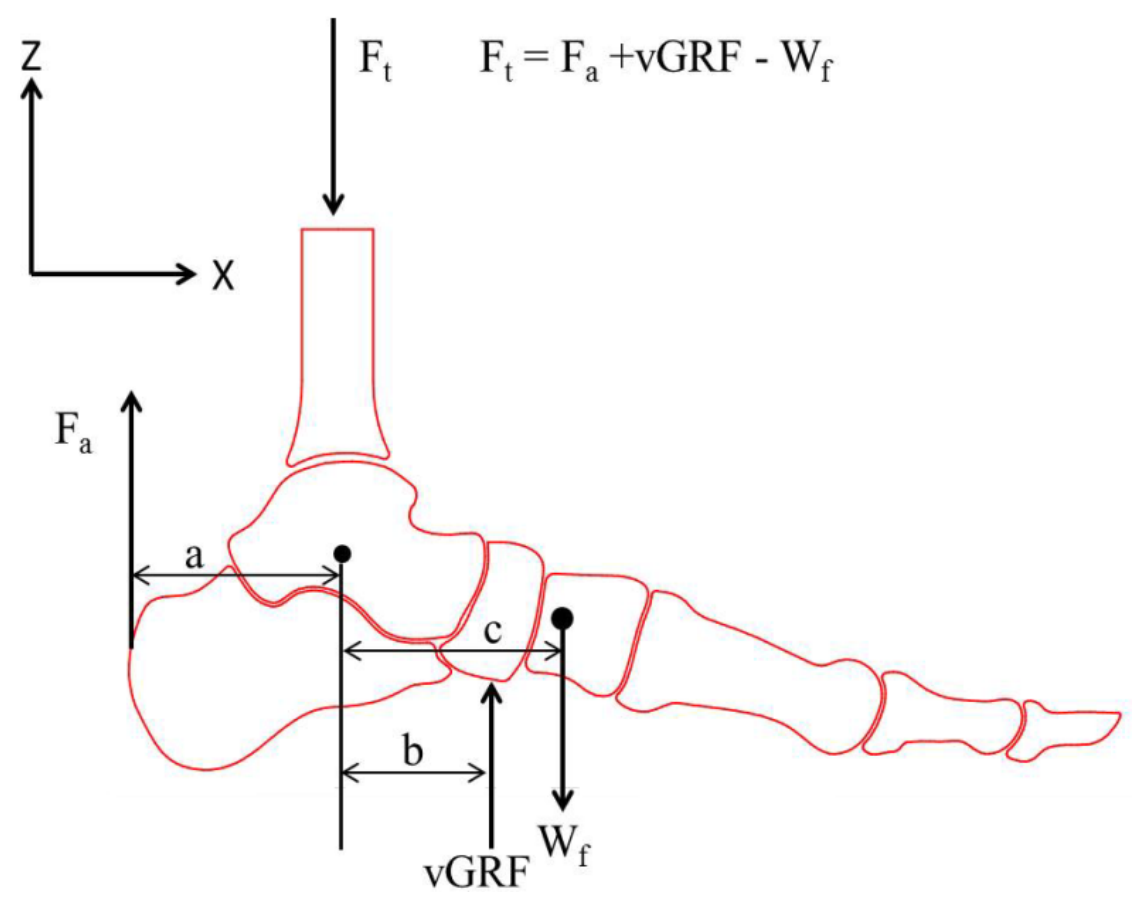

Figure 2-9. Free Body Diagram of Force Vectors and Moment Arms.

Force analysis of the foot and ankle used to set the parameters of loading, where $F_{t}$ represented the axial tibia load, $\mathrm{F}_{\mathrm{a}}$ was the AT load, vGRF was the vertical ground reaction force, and $\mathrm{W}_{\mathrm{f}}$ was the weight of the foot and tibia. The black dots represent an initial prescribed axis of rotation and foot center of mass. A, b, and c represent the respective moment arms associated with these force vectors. Source: Modified with permission: Stewart, T.E., (2012). An Innovative Testing Protocol to Study Foot and Ankle Kinetics during Early Stance Phase of Gait. Master's Thesis, UTHSC. 

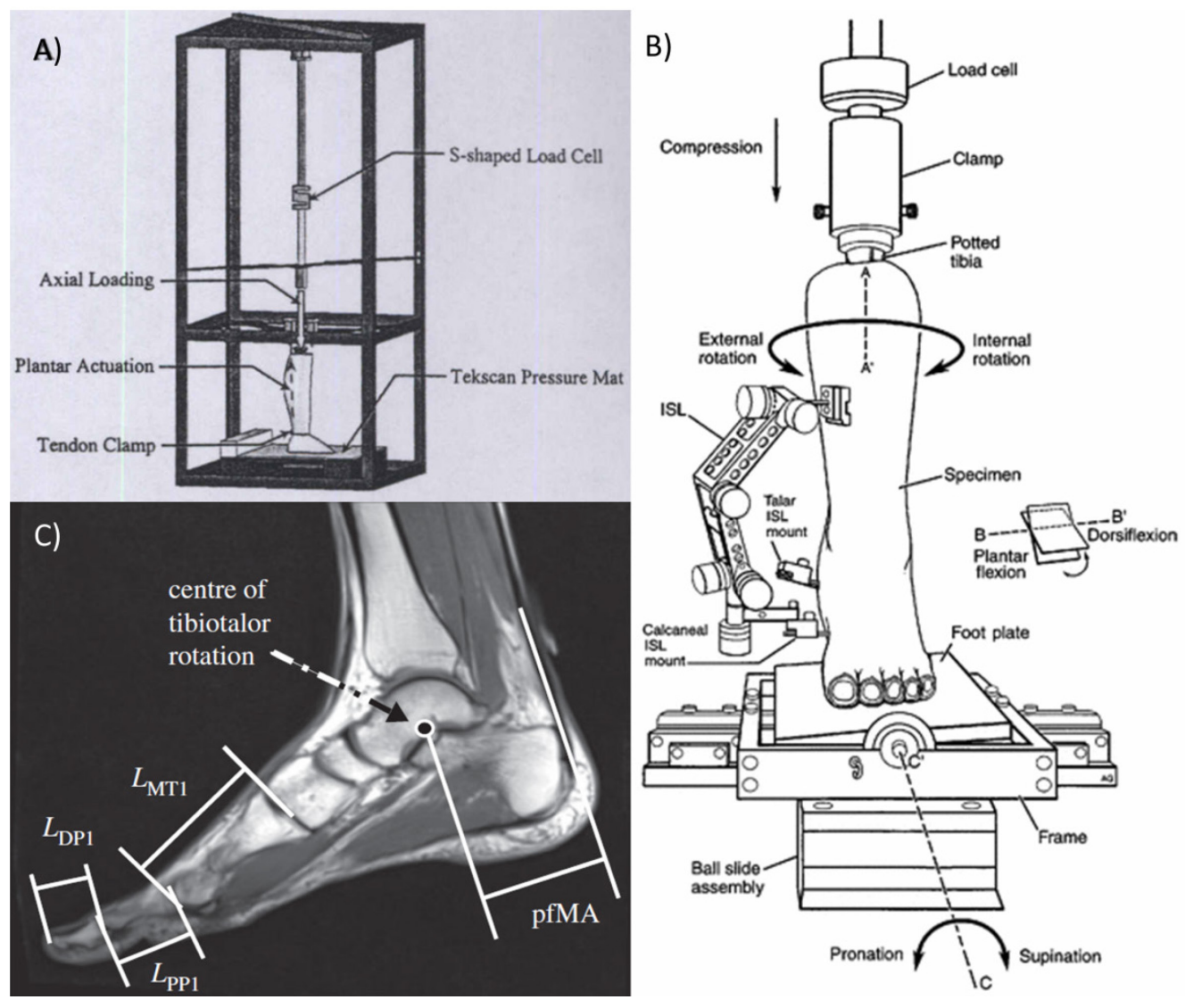

Figure 2-10. BTPs That Investigate a Single Instance of Gait.

A) Design by Jarrell et al. applies a static axial force to the tibia. Source: Reprinted with permission: Jarrell, S.E., Owen, J.R., Wayne, J.S., Adelaar, R.S., 2009. Biomechanical comparison of screw versus plate/screw construct for talonavicular fusion. Foot \& Ankle Intl. 30(2), 150-156. B) Design by Bahr et al. applies axial tibia forces and tilts the foot plate to simulate dorsiflexion, plantarflexion, pronation, or supination. The tibia can be twisted to simulate internal and external rotation. Forces are applied at single instances of gait, usually at large angle intervals. Source: Reprinted with permission: Bahr, R., Pena, F., Shine, J., Lew, W.D., Engebretsen, L. (1998). Ligament force and joint motion in the intact ankle: a cadaveric study.Knee Surg Sports Traumatol Arthrosc. 6(2), 115-21. C) Baxter et al. performed in vivo studies where specimens were asked to tense muscles and rotate the ankle to simulate a single instance of gait. Large angle intervals were used to determine the center of rotation with high error. Source: Reprinted with permission:Baxter, J.R., Novack, T.A., Van Werkhoven, H., Pennell, D.R., Piazza, S.J. (2012). Ankle joint mechanics and foot proportions differ between human sprinters and non-sprinters. Proc Biol Sci. 22(1735), 2018-2024. 

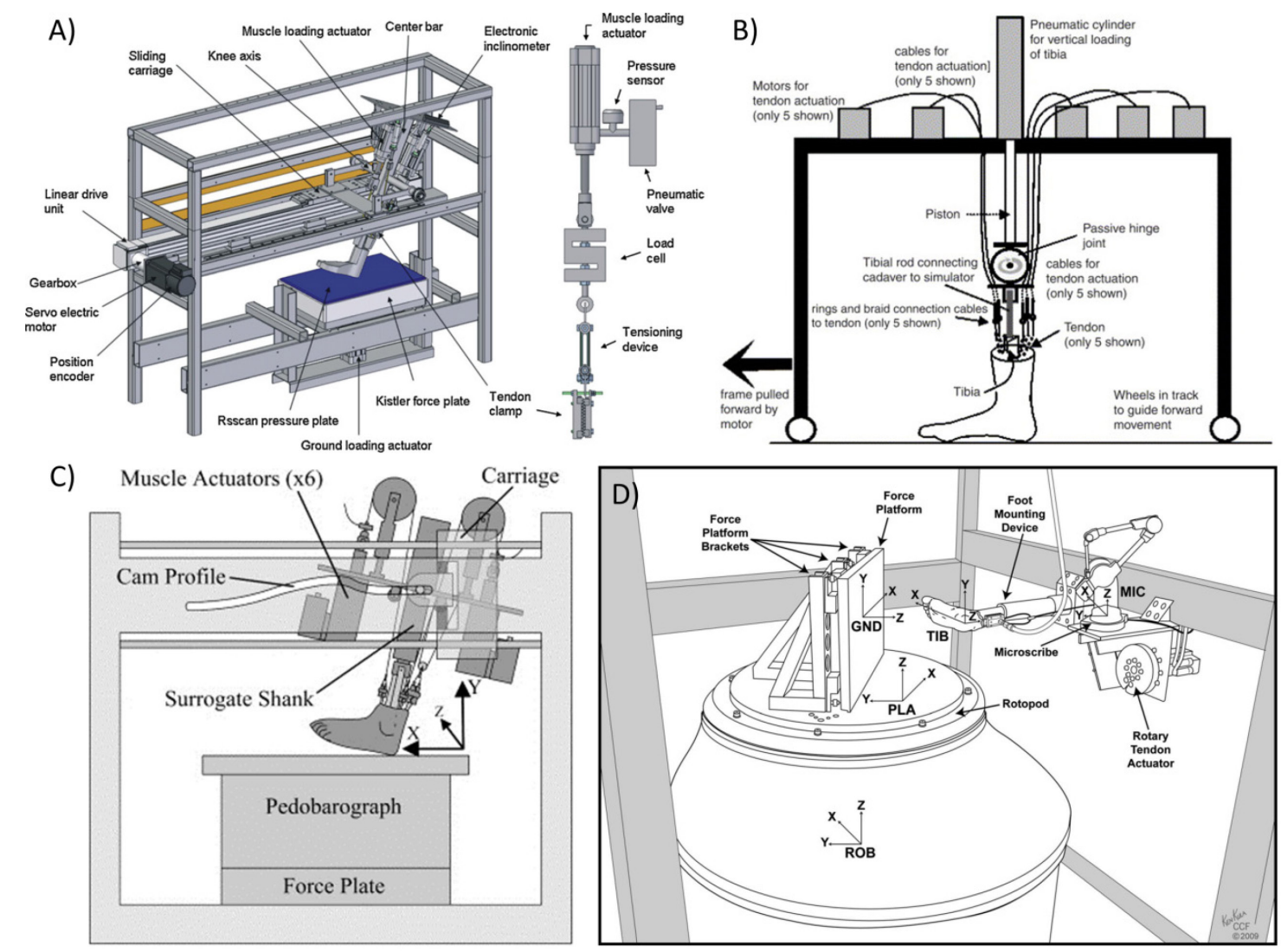

Figure 2-11. Gait Simulators Engineered to Study Foot and Ankle Biomechanics. Each simulator forces a kinematic profile from foot contact to toe off and applies dynamic tendon loads with no ability to measure the IAR. A) Design by Burg et al. applies a dynamic AT load and kinematic profiles determined by a single specimen. Source: Reprinted with permission: Burg, J., Peeters, K., Natsakis, T., Deretmaeker, G., Sloten, J.V. (2012). In vitro analysis of muscle activity illustrates mediolateral decoupling of hind and mid foot bone motion. Gait \& Posture. B) Design by Nester et al. applies dynamic loads to 6 tendons. Source: Reprinted with permission: Nester, C.J., Liu, A.M., Ward, E., Howard, D., Cocheba, J., Derrick, T., Patterson, P. (2007). In vitro study of foot kinematics using a dynamic walking cadaver model. J. Biomech. 40(2), 1927-1937. C) Design by Sharkey and Hamel applies dynamic loads to six tendons and forces a kinematic profile determined by a single specimen. Source: Reprinted with permission: Sharkey, N.A., Hamel, A.J. (1998). A dynamic cadaver model of the stance phase of gait: performance characteristics and kinetic validation. Clin. Biomech. 13, 420433. D) Design by Noble and Colbrunn applies a dynamic AT load. Source: Reprinted with permission: Noble Jr., L.D., Colbrunn, R.W., Lee, D.G., van den Bogert, A.J., Davis, B.L. (2010). Design and validation of a general purpose robotic testing system for musculoskeletal applications. J Biomech Eng. 132(2), 025001:1-12. 


\section{CHAPTER 3. A NOVEL KINEMATIC BASED PROTOCOL TO STUDY FOOT AND ANKLE BIOMECHANICS}

\section{Introduction}

Due to its inherent complexity and physical significance in human activities, the ankle complex is of great importance to researchers of degenerative diseases, injury prevention, and rehabilitation [1,2,3,4,10,27]. In vivo testing [3,35,36,37,38,55] and in vitro studies [14-25,26,27], as well as computational modeling [31-34] have offered valuable insight into the mechanics of the ankle complex. However, each technique possesses some inherent limitations. In vivo studies lack accurate and repeatable measurements [50] and computational modeling lacks the inclusion of soft tissue structures $[11,31]$. In contrast, in vitro studies complement both techniques to produce clinically relevant data, but are limited by current biomechanical testing platforms (BTP) $[14-25,27,35,38]$.

Very few BTPs offer a physiologic loading environment $[15,17,18,19]$, some only investigate one instance of gait [24,27,35,38], and others apply loads or force a kinematic profile estimated by a single specimen $[15,17,20,21]$. Some models assume the ankle complex behaves like a hinge joint with a single axis of rotation, whereas other studies suggested a fixed axis of rotation with articular congruence may be an incorrect kinematic model for ankle joint motion $[11,30,31,36]$. To date, few studies have been conducted that address an instantaneous axis of rotation (IAR) analysis of the ankle joint $[23,35,38]$. IAR data provide additional parameters for characterizing tibiotalar joint motion, where measurements are a direct representation of the effects of joint articular geometry and soft tissue structures. Quantifying the IAR of the ankle joint during gait may have the potential to advance the understanding of the biomechanical properties of the foot and ankle, including arch formation and effects of orthotics and footwear.

There is a need for a biomechanical testing platform and protocol that will provide simulation of controlled tibia and Achilles tendon (AT) loads without constraining the foot-ankle kinematic profile. The objectives of the current work were to: (1) Develop a novel loading protocol that simulated in vivo kinetics of the foot and ankle complex in a human cadaveric lower extremity model during early stance phase gait and (2) Establish an accurate and repeatable measurement of the IAR of the foot-ankle complex.

\section{Materials and Methods}

\section{Tissue Preparation}

A matched pair (male, age 37) of human below knee lower extremity specimens was procured from Restore Life USA (Johnson City, TN). Specimens were frozen at - 
$20^{\circ} \mathrm{C}$. Before preparation, the feet were thawed in a refrigeration system for two days. Medial and lateral radiographs were used to verify the absence of anatomical abnormalities or surgery (see Appendix A for individual specimen radiographs and comparisons). Soft tissue and muscle were resected to expose approximately $100 \mathrm{~mm}$ of the proximal shafts of the tibia and fibula. A single one inch \#6 wood screw was then placed across the proximal ends of the tibia and fibula to stabilize the bones and add additional fixation for the potting material. After the AT was exposed and soft tissue was excised to allow approximately $150 \mathrm{~mm}$ of AT length for clamping, a cable puller was attached and secured with a U-bolt to increase clamping power. The tibial shafts were then mounted concentrically into a cylindrical pot using an alignment frame to position the tibia in a neutral vertical orientation. Low-melting-point bismuth alloy (Rotometals Inc., San Leandro, CA) was used to fix the tibia and fibula together and create a mounting fixture for attachment to the robotic testing platform (RTP). The final prepared specimen is shown in Figure 3-1.

\section{Robotic Testing Platform}

An existing custom designed, multi-axis testing platform was utilized to simulate early stance phase gait under displacement control and force feedback (Figure 3-2) $[54,56]$. Two linear actuators, a Parker Hannifin Corp (Cleveland, OH) 406XR series linear ball screw actuator and an Exlar (Chanhassen, MN) GSX-30 linear roller screw actuator were aligned in the $\mathrm{X}$ and $\mathrm{Z}$-axes, respectively. Platform axes were controlled to within $2 \mu \mathrm{m}$ in the $\mathrm{X}$ and $0.31 \mu \mathrm{m}$ in the $\mathrm{Z}$. The rotary motors and drive units of the original test RTP were upgraded with Harmonic Drive units (Peabody, MA: model FHA25C-160-US250) having improved resolutions of 0.008 degrees. Two six-axis load cells (JR3 Inc., Woodland, CA: models 100M40 and 45E15S) were attached to the RTP assembly and measured the three orthogonal forces and moments applied to the tibia via the gimbal assembly and forces transferred through the foot to the ground at the base of an $\mathrm{X}-\mathrm{Y}$ table. Force capacities and resolutions were $800 \mathrm{~N}$ and $0.44 \mathrm{~N}$ for the gimbal load cell and $4400 \mathrm{~N}$ and $0.52 \mathrm{~N}$ for the base load cell, respectively.

Potted specimens were clamped securely in a mounting block and rigidly connected to the RTP. A sagittal plane was established for each specimen by bisecting the second metatarsal and AT [30,31,37] with a vertical axis aligned with the tibia that matched the X-Z plane of the RTP. A static AT force vector $\left(F_{a}\right)$ was applied via a frictionless cable-pulley system to within four degrees of the vertical tibia axis, while the heel was raised approximately 50mm and the X-Y table was unlocked (Figure 3-3). This allowed for unconstrained arch formation while properly transferring loads from the tibia to the calcaneus and maintaining foot support throughout the gait simulation $[13,26]$.

The heel was then returned to the neutral position and an axial tibia force $\left(\mathrm{F}_{t}\right)$ was applied to the specimen to meet the desired vertical ground reaction force (vGRF) condition within a prescribed $\pm 2 \mathrm{~N}$ tolerance. After the desired loading condition was met, the X-Y table was locked and the rotation of the specimen was started. 


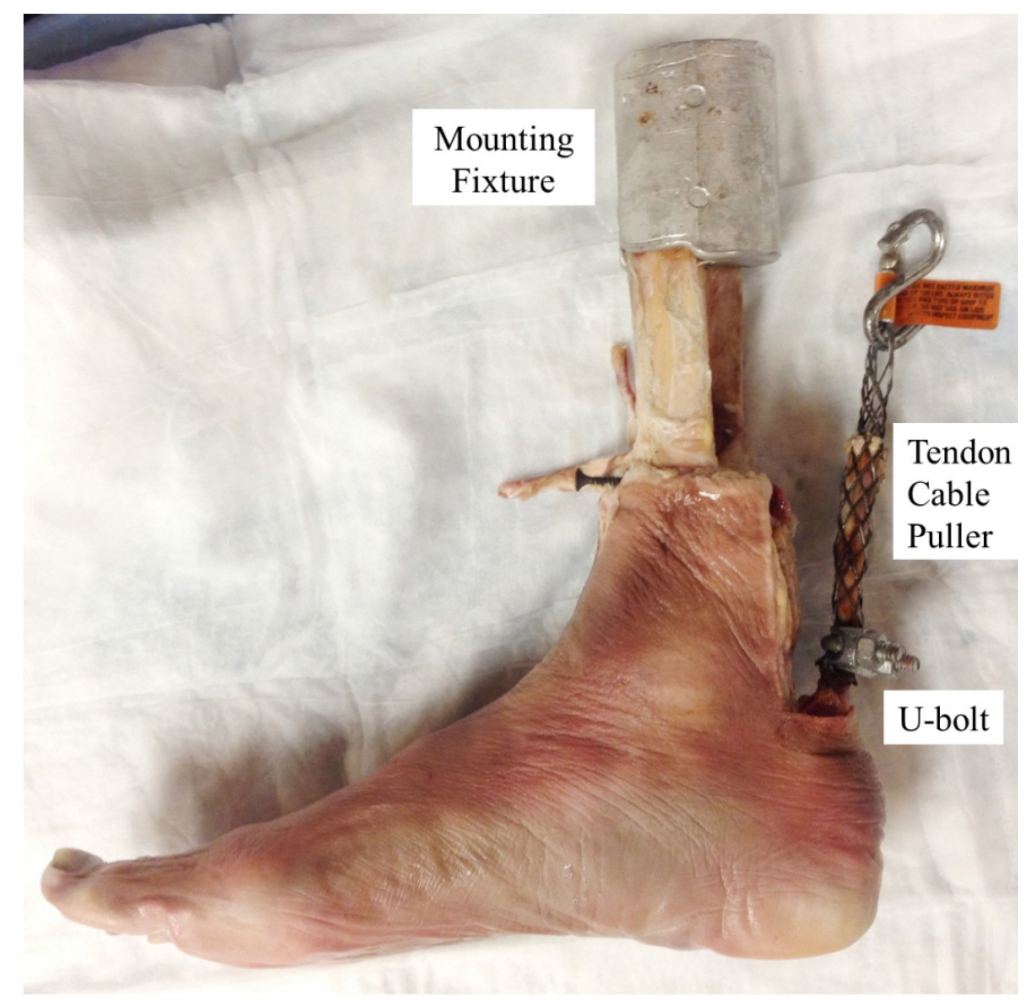

Figure 3-1. A Prepared below the Knee Lower Extremity Specimen.

Tissue was resected from the proximal shafts of the tibia, fibula, and AT. A cable puller was attached to the AT for tendon load application. Bismuth alloy material was used to create a mounting fixture for the proximal shafts of the tibia and fibula for rigid fixation to the testing platform. 


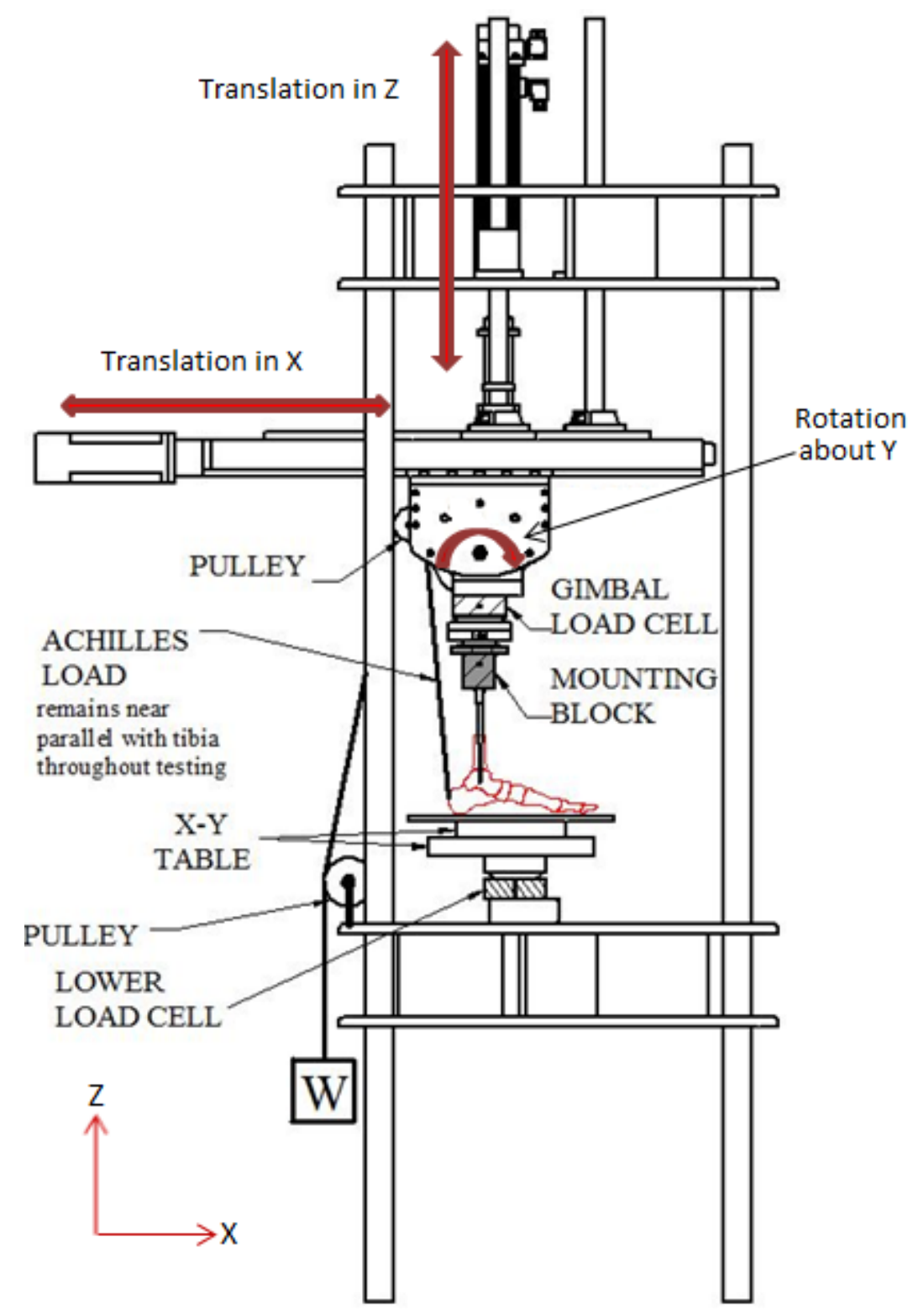

Figure 3-2. Diagram of 4-DOF Robotic System Configured for the Ankle Study. The testing platform consisted of an upper gimbal assembly with two translational and one rotational axes along with a 6-axis load cell, a mounting block to rigidly affix the specimen to the RTP, a pulley system to apply a static load to the AT, and an X-Y table with a second 6-axis load cell to aid in the mounting procedure. The coordinate system is shown by X-, Y-, and Z-axes. Translational and rotational vectors of the controlled axes of the RTP are also shown. Source: Reprinted with permission: Stewart, T.E., (2012). An Innovative Testing Protocol to Study Foot and Ankle Kinetics during Early Stance Phase of Gait. Master's Thesis, UTHSC. 


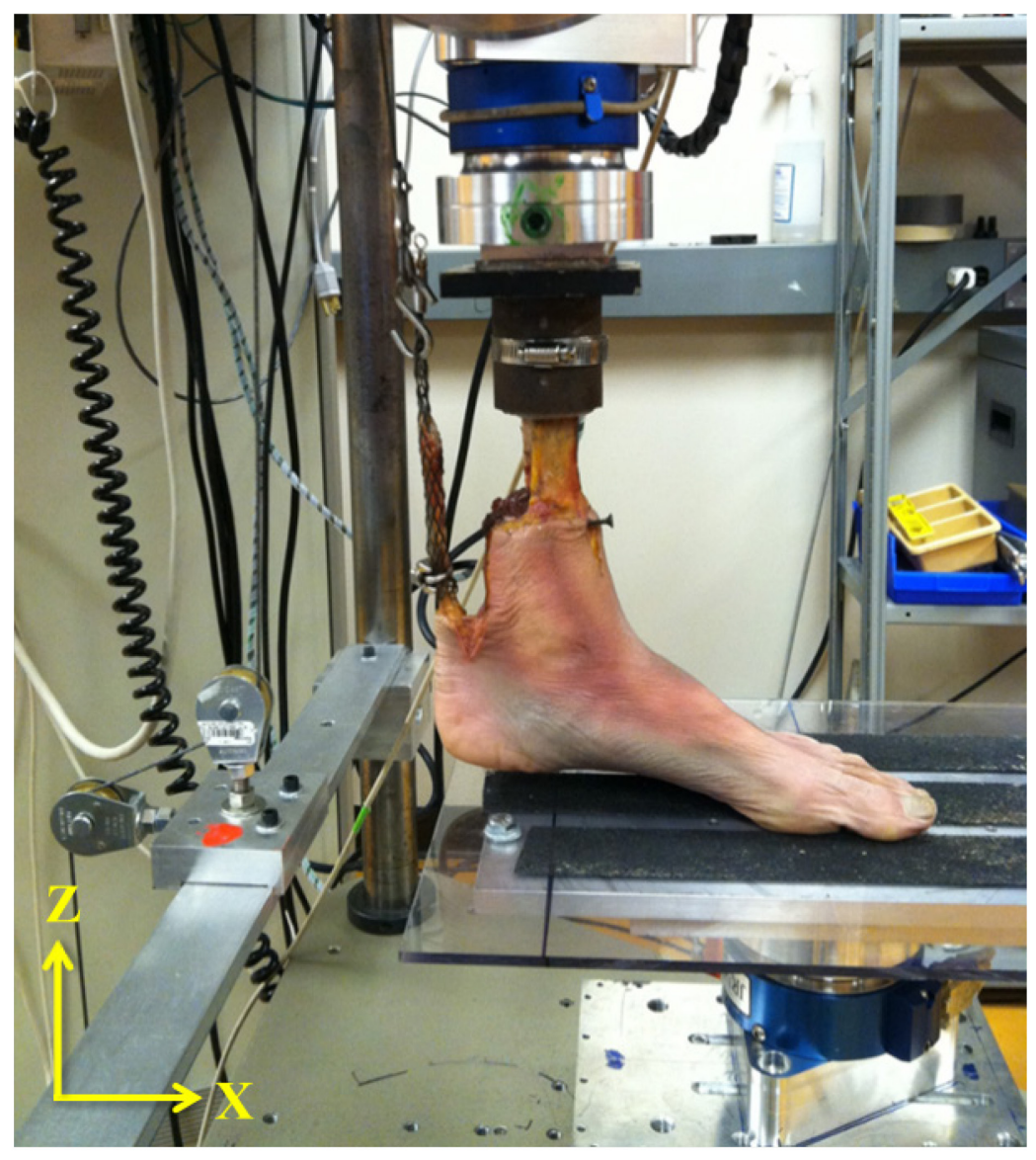

Figure 3-3. Mounting the Specimen.

Specimen mounted in the RTP. This image shows clearance between the heel and X-Y table as the AT load is applied, allowing unconstrained arch formation. 


\section{Test Protocol}

Custom software (Adept Inc., San Jose, CA) was written to implement a "pure moment" strategy to determine the position of the ankle joint for a given angular loading condition. A rigid body analysis was used to describe the dynamic loading characteristics of the ankle as a function of an AT force $\left(\mathrm{F}_{\mathrm{a}}\right)$ and vGRF (Figure 3-4). Using this approach, the AT was statically loaded as a percentage of the vGRF acting on the foot and ankle set at half body weight $(356 \mathrm{~N})$ and a 50\% vGRF $(178 \mathrm{~N})$ AT load was applied based upon values of physiologic peak contractive tension of the AT [37-39]. These conditions were selected within the $800 \mathrm{~N}$ capacity of the upper load cell to achieve reproducible, subinjurious loading conditions.

The tibia started in a neutral orientation ( 0 degrees) and was rotated about an initial prescribed ankle rotational axis (pARA) (Figure 3-5A) that was measured off of a scaled medial radiograph of the foot processed in ImageJ (NIH). This transformation in the $\mathrm{X}$ and $\mathrm{Z}$ directions from the null tool tip (NTT), located at the center of the gimbal, changed the location of the ankle rotational axis (ARA) to the center of the talus, referred to as the extended tool tip (ETT) (see Appendix A for individual ETTs). Because the tibia was rigidly connected to the RTP, the NTT data remained aligned with the rotated axes of the tibia.

The next step in the test protocol was to establish the kinematic path of the ankle joint under pure rotational loading. This was accomplished by introducing an incremental rotation to the specimen and then reducing the off-axis forces by minimizing the distance $(\Delta X, \Delta Z)$ between the pARA and the true ARA (Figure 3-5B), thus a "pure moment" loading condition was achieved. For each incremental rotation, the pure moment loading state could be modified to allow additional shear and compressive forces to be added and directed along the new rotational axes. The process was repeated every 0.5 degrees until the full 10 degrees of dorsiflexion or 20 degrees of plantarflexion was achieved. Five cycles were allotted for conditioning of the tissue followed by five cycles to assess repeatability. Throughout the testing sequence, specimens were kept moist at regular intervals with a $0.9 \%$ saline mist.

\section{Data Analysis}

Force and positional data were sampled at $20 \mathrm{~Hz}$. A modified set of equations originally derived by Crisco et al (1994) were applied to the NTT rotational and positional data for each increment of rotation to calculate the IAR of the ankle within $\pm 0.001 \mathrm{~mm}[53]$. An "ankle coordinate frame" for the IAR was a fixed reference frame for tibial motion about the ankle joint and was defined by the bisector of the tibia and highest point on the tibial mortise in the initial neutral vertical orientation (Figure 3-6) (See Appendix A for more detailed description). IAR paths were plotted in Excel for each specimen.

The test protocol had each specimen cycled five times for tissue conditioning and 


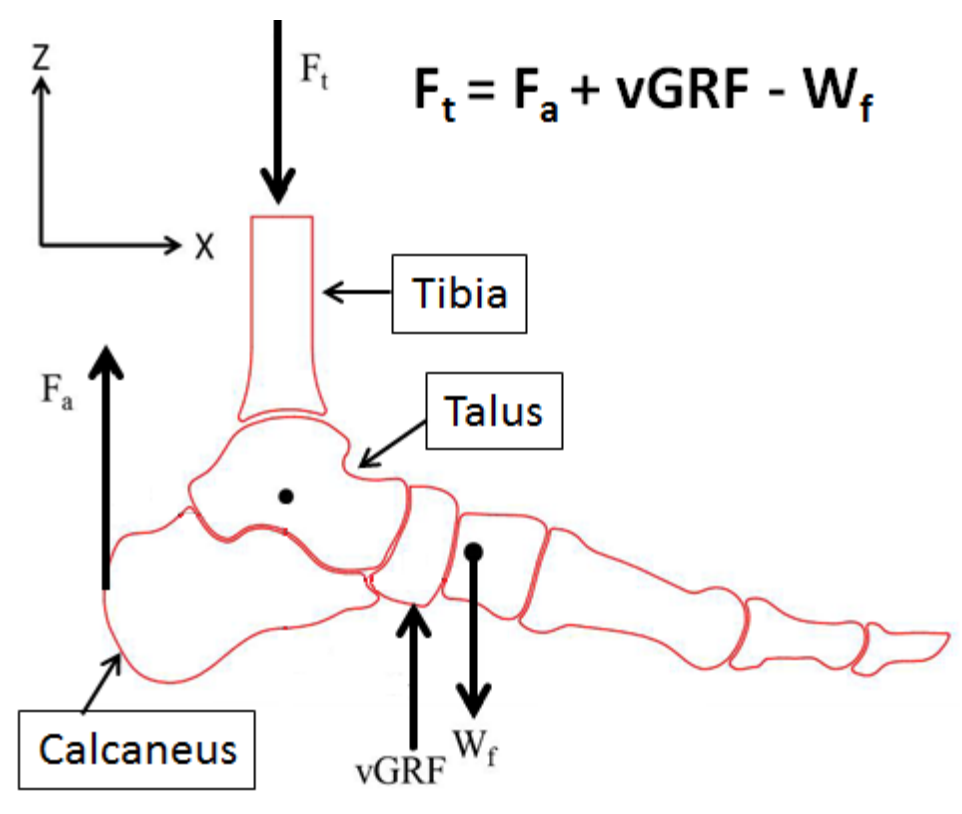

Figure 3-4. Force Analysis of the Foot and Ankle Used to Set the Parameters of Loading.

$F_{t}$ represented the axial tibia load, $F_{a}$ was the AT load, vGRF was the vertical ground reaction force, and $\mathrm{W}_{\mathrm{f}}$ was the weight of the foot and tibia. The black dots represent an initial prescribed axis of rotation and foot center of mass. 
A)

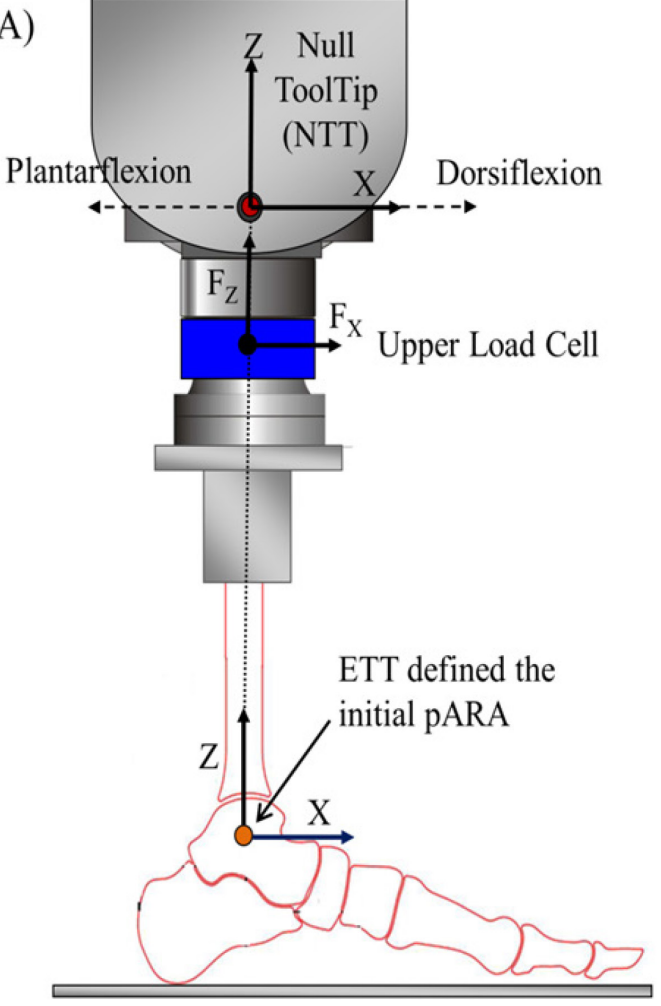

B)

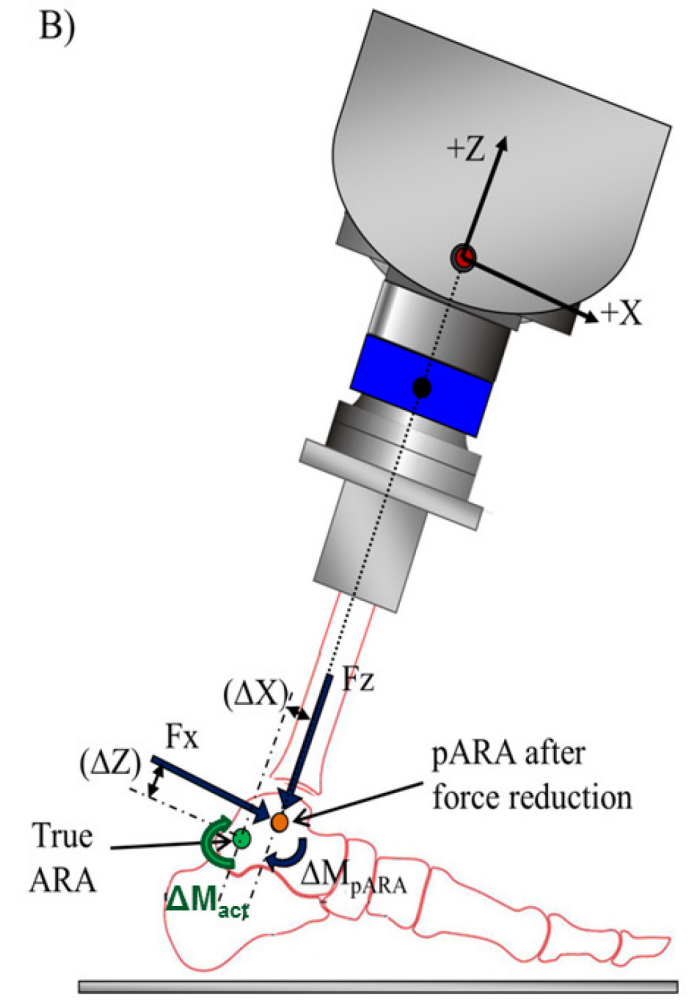

Figure 3-5. Modified "Pure Moment" Program.

Kinematic and load control strategy. A) Kinematic coordinate frames of the foot and ankle are defined by the NTT and ETT of the RTP. The ETT represents the initial prescribed IAR of the foot located at the center of the talus and was a negative $\mathrm{Z}$ offset from the NTT. The coordinate frame of the load cell was initially located at the center of the load cell and transformed to the origin of the moving joint center, i.e., the ETT. B) An angular input of ankle rotation was given every 0.5 degrees, during which the loading protocol reduced the ankle moment due to off-axis forces by minimizing the distance $(\Delta \mathrm{X}, \Delta \mathrm{Z})$ between the pARA (upper) and the true ARA (lower), thus creating a "pure moment" condition. Additional shear and compressive loads could be introduced at this time to maintain the axial tibial force and vGRFs. The NTT data remained aligned with the rotated tibia axes. Achilles load is not shown. 


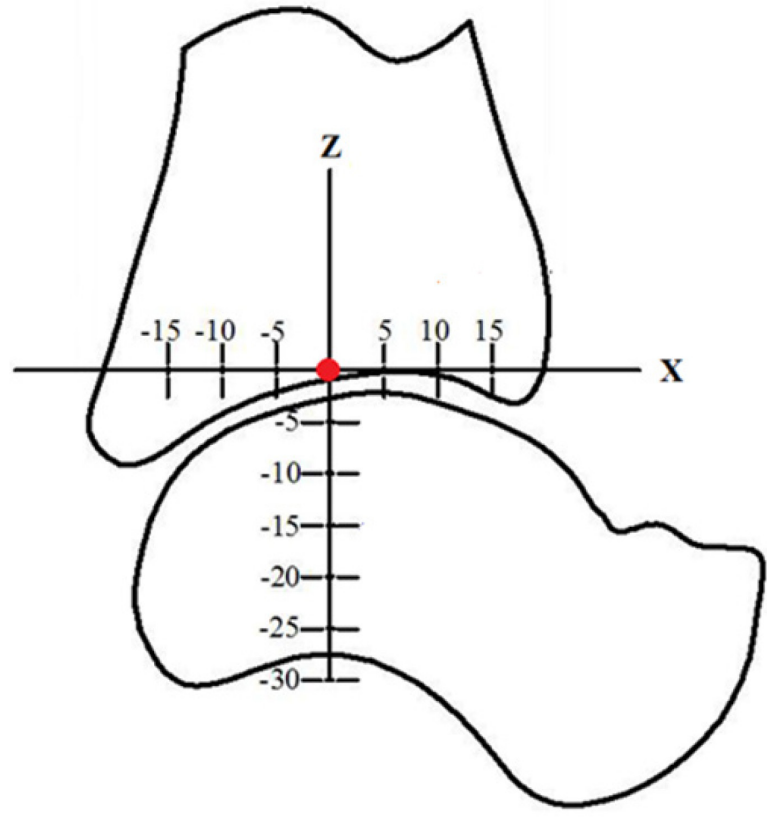

Figure 3-6. The "Ankle Coordinate Frame."

Designated to plot the IAR axis on different specimen radiographs. It is defined as the intersection of bisector of the tibia and the highest point on the tibial mortise. 
five times in dorsiflexion and plantarflexion to demonstrate repeatability. The final three cycles were plotted in Excel and analyzed statistically using GraphPad Prism 6.0 software (La Jolla, CA). Mean axial tibia forces and mean X- and Z- IAR values were compared separately for dorsi- and plantar-flexion using a One Way ANOVA with a Holm Sidak post hoc test for multiple comparisons $(\alpha=0.05)$ to show variability between cycles. The mean X-and Z- IAR values for all three cycles were also compared between dorsi- and plantar- flexion. Additionally, the slopes of the plantarflexion IAR paths were compared between cycles using a linear regression analysis in Graphpad Prism 6.0 software. Results are presented as means \pm standard deviations. A $P$-value of less than 0.05 signified significant differences between comparisons.

\section{Results}

The mean axial tibia loads throughout full rotation for each specimen are shown

in Table 3-1. No statistical differences were found between cycles of motion $(\mathrm{P}>0.05$ for all comparisons). The maximum tibial force error was typically within the prescribed $\pm 2 \mathrm{~N}$ tolerance for all tests, with a maximum error of $6 \mathrm{~N}$. These force errors predominantly occurred within the final degrees of motion.

Mean IAR values for specimen 1 and specimen 2 are provided in Tables 3-2 and 3-3, respectively. No statistical differences were found in mean X-and Z- IAR values between cycles of dorsi- or plantar-flexion for either specimen $(\mathrm{P}>0.05$ for all comparisons). When comparing mean IAR values for all three cycles between dorsi- and plantar-flexion, statistically significant values were found in the mean X-IAR values for both specimen $(\mathrm{P}<0.0001)$ and in the mean $\mathrm{Z}$ - IAR values in specimen $2(\mathrm{P}<0.0001)$.

The final 3 cycles of IAR pathways are plotted on their respective radiographs for both specimens with mean IAR values and standard deviations (Figure 3-7). The IAR represents a moving axis of rotation due to the joint articular geometry and surrounding soft tissue structures. Dorsiflexion paths show little movement in the talus, remaining within a $4.5 \mathrm{~mm}$ range (i.e., $2 \mathrm{~mm}$ in the $\mathrm{X}$ and $4 \mathrm{~mm}$ in the $\mathrm{Z}$ ). In plantarflexion, the first degrees of motion started up within the articular surface of the talus. As rotation continued, the IAR path moved downward towards the middle of the talus. The plantarflexion IAR path had a maximum range of approximately $21 \mathrm{~mm}$ (i.e., $20 \mathrm{~mm}$ in the $\mathrm{Z}$ and $6 \mathrm{~mm}$ in the $\mathrm{X}$ ). A linear regression analysis was performed on the plantarflexion paths. No statistical differences were found between the slopes of the IAR paths for specimen $1(\mathrm{P}=0.7823)$ and specimen $2(\mathrm{P}=0.0826)$.

\section{Discussion}

A novel dynamic robotic testing platform and protocol were developed and used to investigate the biomechanical behavior of the foot and ankle under simulated loading conditions representative of in vivo conditions of early stance phase gait. The first objective of this study was to validate the accuracy of a loading protocol. The model 
Table 3-1. Average Axial Tibia Loading Conditions for Specimen between Cycles of Motion.

\begin{tabular}{ccc}
\hline \multirow{2}{*}{ Specimen } & \multicolumn{2}{c}{$\begin{array}{c}\text { Axial Tibia Load }(\mathrm{N}) \\
(\text { Mean } \pm \text { Standard Deviation) }\end{array}$} \\
\cline { 2 - 3 } & Dorsiflexion & Plantarflexion \\
\hline 1 & $516.601 \pm 0.869$ & $506.294 \pm 1.314$ \\
2 & $519.291 \pm 0.721$ & $513.633 \pm 0.427$ \\
\hline
\end{tabular}

Table 3-2. Mean IAR Values for Specimen 1.

\begin{tabular}{|c|c|c|c|c|}
\hline \multirow[t]{2}{*}{ Cycle } & \multicolumn{2}{|c|}{$\begin{array}{c}\text { XIAR }(\mathrm{mm}) \\
(\text { Mean } \pm \text { Standard Deviation })\end{array}$} & \multicolumn{2}{|c|}{$\begin{array}{c}\text { ZIAR }(\mathrm{mm}) \\
(\text { Mean } \pm \text { Standard Deviation })\end{array}$} \\
\hline & Dorsiflexion & Plantarflexion & Dorsiflexion & Plantarflexion \\
\hline 1 & $-2.952 \pm 0.118$ & $1.001 \pm 1.265$ & $-8.982 \pm 0.510$ & $-8.838 \pm 5.178$ \\
\hline 2 & $-2.916 \pm 0.163$ & $0.548 \pm 1.087$ & $-9.401 \pm 0.454$ & $-9.282 \pm 4.661$ \\
\hline 3 & $-2.868 \pm 0.076$ & $1.025 \pm 1.280$ & $-9.184 \pm 0.528$ & $-8.717 \pm 5.374$ \\
\hline Mean & $-2.912 \pm 0.042 *$ & $0.858 \pm 0.269 *$ & $-9.189 \pm 0.210$ & $-8.946 \pm 0.297$ \\
\hline
\end{tabular}

* Statistically significant between dorsiflexion and plantarflexion mean IAR values.

Table 3-3. Mean IAR Values for Specimen 2.

\begin{tabular}{lccccc}
\hline \multirow{2}{*}{ Cycle } & \multicolumn{2}{c}{$\begin{array}{c}\text { XIAR }(\mathrm{mm}) \\
\text { (Mean } \pm \text { Standard Deviation) }\end{array}$} & & \multicolumn{2}{c}{$\begin{array}{c}\text { ZIAR }(\mathrm{mm}) \\
\text { (Mean } \pm \text { Standard Deviation) }\end{array}$} \\
\cline { 2 - 3 } \cline { 5 - 6 } & Dorsiflexion & Plantarflexion & & Dorsiflexion & Plantarflexion \\
\hline 1 & $-4.228 \pm 0.626$ & $-0.231 \pm 1.338$ & & $-6.400 \pm 0.705$ & $-3.532 \pm 4.934$ \\
2 & $-4.309 \pm 0.501$ & $0.088 \pm 1.516$ & & $-6.270 \pm 1.278$ & $-3.081 \pm 6.160$ \\
3 & $-4.297 \pm 0.243$ & $-0.088 \pm 1.470$ & & $-6.666 \pm 0.545$ & $-3.566 \pm 5.272$ \\
Mean & $-4.278 \pm 0.044 *$ & $-0.077 \pm 0.160 *$ & & $-6.445 \pm 0.202 *$ & $-3.393 \pm 0.271^{*}$ \\
\hline
\end{tabular}

* Statistically significant between dorsiflexion and plantarflexion mean IAR values. 


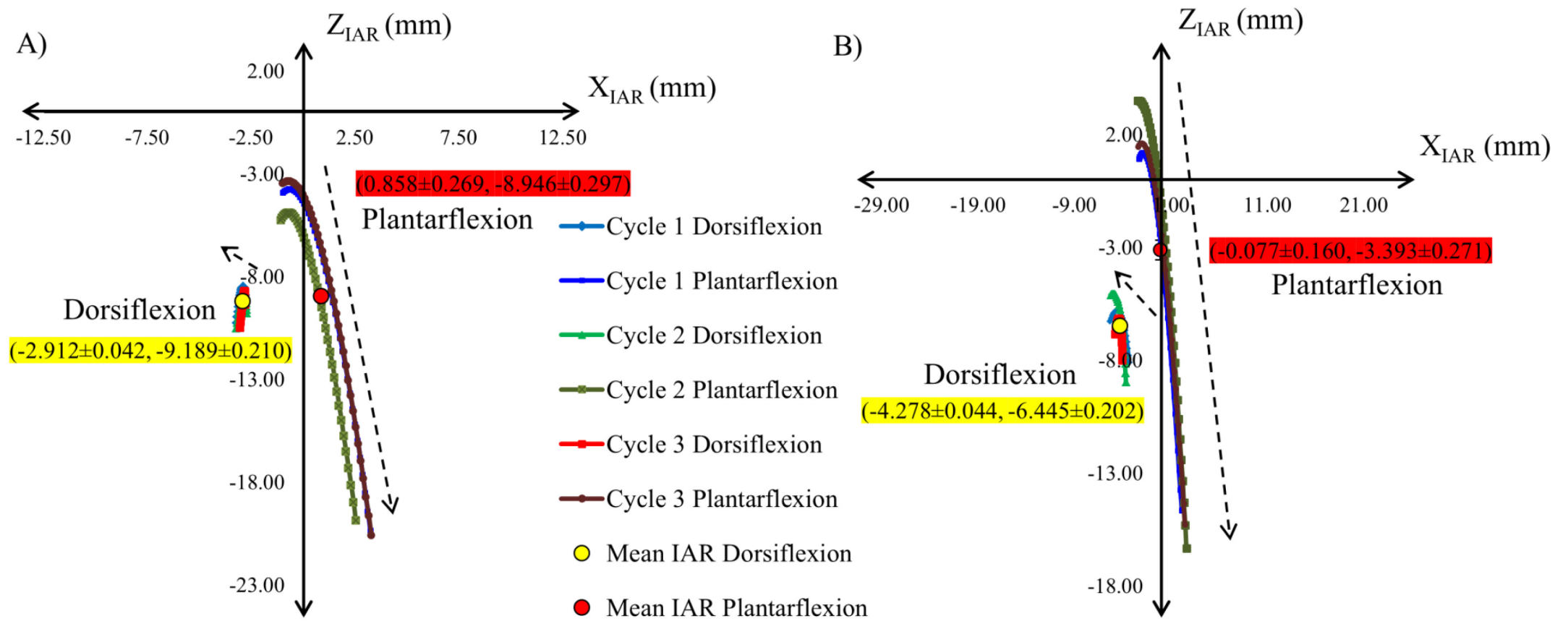

Figure 3-7. IAR Paths.

A) Specimen 1 and B) Specimen 2 during dorsiflexion and plantarflexion plotted on a medial radiographs. Mean IAR values for all three cycles (circles) with standard deviations (too small to see) are shown. Dashed arrows denote the direction of the IAR through rotation. 
simulates a static Achilles load during stance phase of walking with decreased in vivo loading conditions. The role of soft tissue in this model was accounted for by preconditioning of the cadaver feet by subjecting them to several load and movement cycles before relevant data were captured. The simulation accounted for 20 degrees of plantarflexion and 10 degrees dorsiflexion, whereas the total functional range of walking motion of the ankle during stance phase is approximately 15 degrees [3,27,37,39]. While force errors were typically controlled within prescribed $\pm 2 \mathrm{~N}$ tolerances throughout testing, they increased towards the end of motion possibly as a result of rotating the ankle beyond its functional limit. The ATLs were limited by the upper loading capacity of the gimbal load cell, such that loading conditions were set at half body weight $(356 \mathrm{~N})$ and the Achilles tendon force was set to half the vGRF (178N). However, the ground reaction forces produced during the simulation were in agreement with a percentage of recorded in vivo forces $[1-4,11,39]$. Additionally, application of the AT force to within four degrees of the long axis of the tibia introduced a minimal force error of $0.25 \%$.

The second objective of the current study was to address intra-specimen variability between cycles of motion. Stability of the ankle joint is determined by three main factors: articular congruity, ligamentous structures, and ankle position [11,36]. All have been shown to have high variability between specimens [51]. Baxter showed a difference in biomechanical properties of sprinters versus non sprinters, suggesting physical health plays a major role in mechanics of the ankle [35]. Therefore, the goal was to demonstrate a repeatable measurement of the IAR in the sagittal plane. This loading protocol showed variability in the mean IAR measurements between cycles of motion (as measured by the standard deviation) of less than $1 \mathrm{~mm}$. The talus dimension for the two specimens were approximately $60 \mathrm{~mm}$ in width and $38 \mathrm{~mm}$ in height, demonstrating small relative variability on a clinical scale. Current gait simulators and BTP protocols are either unable to measure the IAR [14-22,24] or are limited to high errors in the calculation of the IAR due to methodology $[23,35]$. While dorsiflexion paths remained within a $4.5 \mathrm{~mm}$ range, plantarflexion paths showed more movement. However, plantarflexion slopes were not significantly different between cycles of motion in specimen 1 or 2, demonstrating a repeateable measurement of the IAR path.

Some limitations are present in this study. The proximal fusion of the tibia and fibula removed the physiologic joint function where the bones may undergo translations and rotations relative to each other. This has been observed primarily when the knee experiences external and internal rotation [28]. Our model accounts for tibial sagittal plane motion only. During force application an out of plane load was produced as a mode of lateral stabilization of the joint. However, a negligible moment build up was observed throughout motion $(0.5 \mathrm{Nm})$ and did not restrict flexion. Additionally, the current model does not incorporate a dynamic Achilles tendon force profile representative of in vivo loading conditions, nor does it account for any of the other major plantarflexors, extrinsic dorsiflexors, or intrinsic muscles of the foot. However, these muscles are predominantly active during heel strike, and heel rise to toe off gait $[36,39]$. The force applied via the Achilles tendon has been shown to have the greatest role in the biomechanical behavior of the ankle joint during stance phase when the foot is flat. Therefore, it is the only tendon force accounted for in this study. During normal 
gait, the tibia is driven from maximum plantarflexion to dorsiflexion with a continuous load on the joint. This protocol is limited in that the dorsiflexion and plantarflexion paths are generated independently. Furthermore, plantarflexion is driven in a direction uncharacteristic of the target in vivo stance phase motion. However, the Achilles tendon load is applied to the specimen in such a manner to simulate the in vivo loading scenario where the joint is plantarflexed. The independent movement from the neutral orientation between dorsi- and plantar-flexion may explain the disconnect between the initial point of IAR paths in dorsiflexion and plantarflexion. Another limitation is that our study was two-dimensional whereas the ankle joint can move in three dimensions $[1,11,50]$.

In conclusion, this work has provided description and validation of a novel loading protocol developed to support in vitro cadaveric testing of the foot and ankle complex. This protocol provided a two-dimensional analysis of the IAR of the ankle joint using tightly controlled loads in conjunction with a passive AT force. The low force error tolerance and consistent axial force values demonstrate the RTP's ability to accurately simulate forces. The results of the study are one of a handful to address the instantaneous axis of rotation of the ankle joint and provide the most accurate measurement to date. The IAR measurement was repeatable within one millimeter, smaller than what is feasibly measurable in a clinical setting. Because the IAR measurement is a direct representation of soft tissue structures and articular geometry, future in vitro studies may yield great insight into the biomechanical properties of the foot and ankle within the sagittal plane, including arch formation and effects of orthotics and footwear on ankle kinematics. As new information describing the dynamic loading characteristics of the foot and ankle is obtained from in vivo gait studies (stair climbing, orthotics, footwear), this novel RTP and test protocol can readily simulate these conditions. Current development includes incorporating a dynamic Achilles tendon load, a higher capacity load cell to accommodate forces comparable to in vivo loads, and an optoelectric motion tracking system to study the interaction of the bones of the arch of the foot throughout ankle rotation. 


\section{CHAPTER 4. EFFECTS OF ACHILLES LOADING ON THE KINEMATIC PROPERTIES OF THE FOOT-ANKLE COMPLEX DURING STANCE PHASE GAIT}

\section{Introduction}

Several studies have been conducted to accurately describe bony motion of the foot during normal gait to aid in identification of injury, foot abnormality, surgical correction, and implant design [35,41-49,57,58]. In vivo studies involving X-ray photogrammetic [43], magnetic resonance imaging (MRI) [35,45,47,48], skin mounted retro-reflective markers [41,42,44,46,57], and videofluoroscopy [49] techniques are typically avoided due to: invasiveness, limited field of data measurement, required data reduction, rigid body assumptions, or reduced repeatability of the measurement. Skin mounted retro-reflective markers suffer from inaccuracies due to skin-motion artifacts. Additionally, movement of groups of bones such as the tarsals, navicular, talus, cuboids and cuneiforms are often combined into gross motion of the complex or are ignored due to the invasiveness of accessing the bones.

In vitro bone pin analyses used in cadaveric gait models offer a more accurate measurement of foot kinematics, but are subject to the limitations of current biomechanical testing platforms (BTPs). Some of these limitations are: applying a nonphysiologic loading environment [15-19,46], using simplified tibial kinematics $[15,17,20,22,29,46]$, or lacking dynamic loading conditions and tendon loads $[17,24,25,27,35]$. Additionally, BTPs lack the ability to measure the instantaneous axis of rotation (IAR). The IAR represents a more complete parameter for characterizing joint motion. The ability to detect shifts in IAR may help in defining injury type and/or the impact of injury on foot ankle mechanics, as well as the effects of surgical procedures and implant and orthotic design. While most kinematic analyses of the foot are characterized by simple rotations, joint motion can also be described by rolling and sliding of articular surfaces during motion with a dependency on loading scenarios [31]. Load plays a major role in ankle biomechanics including influences on the articular surfaces, bony motion, and formation of the arches [51]. Arch formation is key in supplying a rigid structure to transfer loads from the tibia to the ground. Activated primarily by the Achilles tendon (AT), it is suggested that once the arch is formed, little motion occurs between the calcaneus, navicular, and talus throughout stance phase $[20,25,36,44,46,58]$. This suggests most of the motion in the foot and ankle remains between the articular surfaces of the tibia and talus. However, studies concerning the effects of joint loading are limited. Paired with a bony motion analysis, a study incorporating an IAR measurement would offer the most complete description of the kinematics of the foot and ankle. Thus a need exists for a more physiologic and accurate BTP that would enable accurate calculation of the ankle IAR under different loading conditions.

We began to address these concerns by using an existing kinematic based protocol to study foot and ankle biomechanics in a cadaveric lower extremity model that provided 
a complete description of the bony motion and IAR of the foot during stance phase gait. The aim of the study was to demonstrate the effect of active and passive loading conditions as well as a variable Achilles load (25\%, 50\%, 75\%, 100\% body weight) on the IAR of the ankle joint as well as the bony motion of the talus, calcaneus, and navicular throughout motion. We hope this study will illuminate the effects of an Achilles load on arch formation by showing whether the arch remained a rigid structure for the different loading conditions and whether ankle motion was restricted due to arch formation.

\section{Materials and Methods}

\section{Specimen Preparation}

Four fresh-frozen cadaveric lower limb specimens (1 female pair, age 44, 1 male pair, age 37) were procured from Restore Life USA (Johnson City, TN). Before preparation, the feet were thawed in a refrigeration system for two days. Each specimen was screened radiographically by an orthopaedic surgeon to exclude the presence of any anatomical abnormalities (see Appendix A for individual specimen radiographs and comparisons).

Soft tissue and muscle were resected to expose approximately $100 \mathrm{~mm}$ of the proximal shafts of the tibia and fibula. The tibia and fibula were fixed proximally with a single one inch \#6 wood screw. The AT was exposed and soft tissue was excised to allow approximately $150 \mathrm{~mm}$ for clamping using a cable puller secured with a U-bolt. Specimens were then mounted concentrically into a cylindrical pot using an alignment frame to position the tibia in a neutral vertical orientation. Low-melting-point bismuth alloy (Rotometals Inc., San Leandro, CA) provided final fixation of the tibia and fibula. Four standard Kirschner wires (K-wires) were inserted into the talar neck, navicular, and calcaneus under X-Ray guidance with caution to avoid tendon structures. Tissue was resected around K-wire insertion areas to reduce obstruction of K-wire motion during testing.

\section{Robotic Testing Platform and Protocol}

An existing custom designed, multi-axis testing platform was utilized to simulate early stance phase gait under displacement control and force feedback (Figure 4-1A) $[54,56]$. Two linear actuators, a Parker Hannifin Corp (Cleveland, OH) 406XR series linear ball screw actuator and an Exlar (Chanhassen, MN) GSX-30 linear roller screw actuator were aligned in the $\mathrm{X}$ and $\mathrm{Z}$-axes, respectively. Platform axes were controlled within 2 microns in $X$ and 0.31 microns in the $Z$. The rotary motors and drive units of the original test RTP were upgraded with Harmonic Drive units (Peabody, MA: model FHA25C-160-US250) having improved resolutions of 0.008 degrees. Two six-axis load cells 


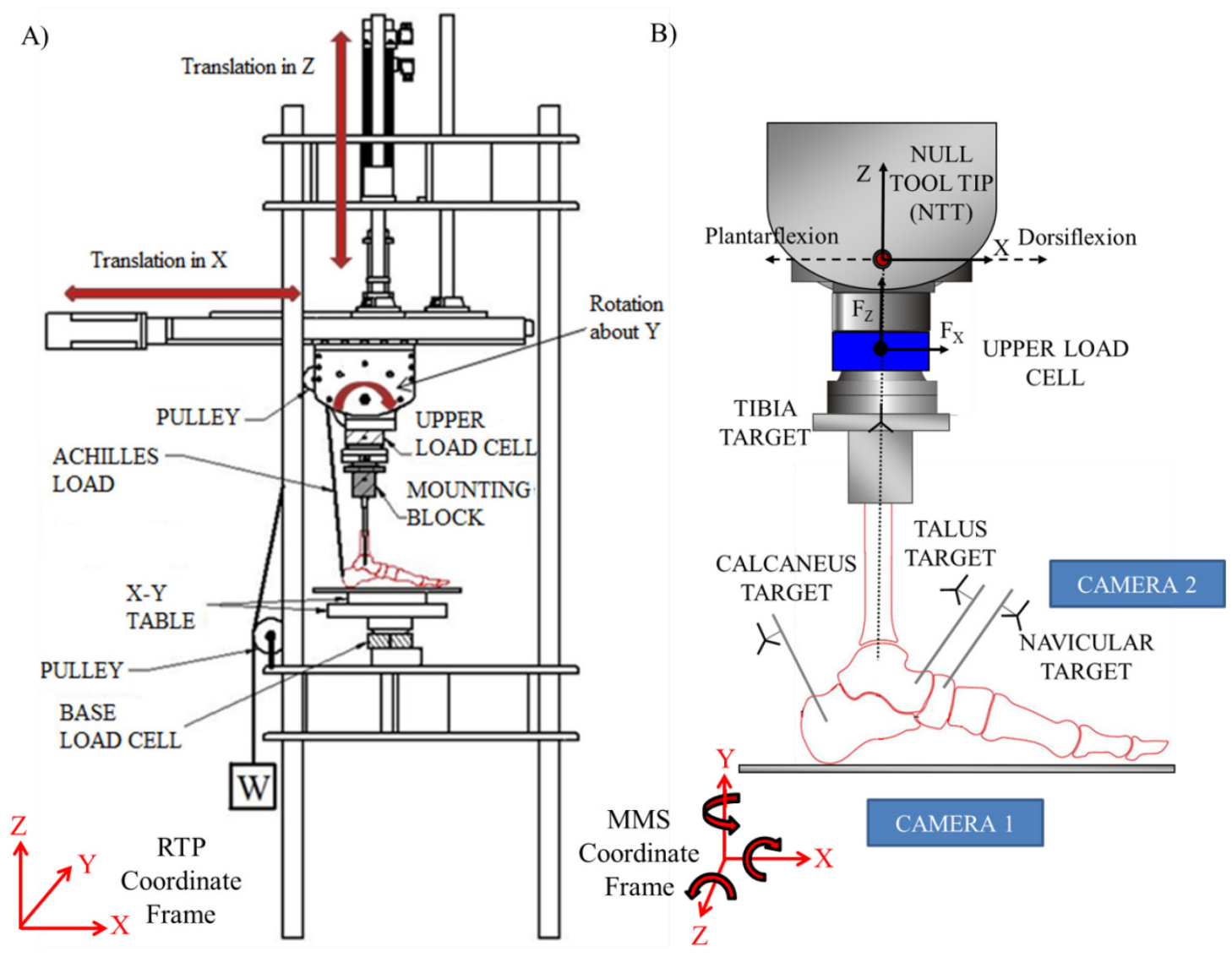

Figure 4-1. Diagram of 4-DOF Robotic System Configured for the Ankle Study. A) The testing platform consisted of an upper gimbal assembly with two translational and one rotational axes along with a 6-axis load cell, a mounting block to rigidly affix the specimen to the RTP, a pulley system to apply a static load to the AT, and an X-Y table with a second 6-axis load cell to aid in the mounting procedure. The coordinate system is shown by X-, Y-, and Z-axes. Translational and rotational vectors of the controlled axes of the RTP are also shown. Source: Reprinted with permission: Stewart, T.E., (2012). An Innovative Testing Protocol to Study Foot and Ankle Kinetics during Early Stance Phase of Gait. Master's Thesis, UTHSC. B) MMS diagram. Cameras were aligned in the sagittal plane 180 degrees apart. Three dimensional optoelectric LED target arrays were mounted on K-wires implanted in the talus, calcaneus, and navicular, and one was placed on the mounting block as reference to tibial motion. Targets aligned with orientation of the K-Wire. The MMS coordinate system is shown with associated rotations. 
JR3 Inc., Woodland, CA: models 67M25S3 and 45E15S) were attached to the RTP assembly and measured the three orthogonal forces and moments applied to the tibia via the gimbal assembly and forces transferred through the foot to the ground at the base of an $\mathrm{X}-\mathrm{Y}$ table. Force capacities and resolutions were $1157 \mathrm{~N}$ and $0.29 \mathrm{~N}$ for the gimbal load cell and $4400 \mathrm{~N}$ and $0.52 \mathrm{~N}$ for the base load cell, respectively.

An optoelectric motion measurement system (MMS) (Dynasight ${ }^{\mathrm{TM}}$, Origin Instruments, Texas) was used to measure translations and rotations of bone structures (Figure 4-1B). Three-dimensional optoelectric target arrays were mounted onto the implanted K-wires in the foot and one target remained in the mounting fixture as a reference to tibial rotation. Targets were aligned with the orientation of the K-wires. This allowed a direct, repeatable measurement of the placement of the target as well as the orientation of the K-wires relative to the sagittal plane (Appendix B). The X, Y, and $\mathrm{Z}$ axes of the MMS were aligned with the $\mathrm{X}, \mathrm{Z}$, and $\mathrm{Y}$ axes of the RTP, respectively. These coordinate systems were aligned with anatomical cardinal planes and do not necessarily reflect the axes of rotation of joints. Bone rotation and positional data were collected three times at $10 \mathrm{~Hz}$ during playback of the fifth cycle of motion.

A sagittal plane was established by bisecting the second metatarsal and AT with a vertical axis aligned with the tibia that matched the X-Z plane of the RTP [30,31,37]. Static AT force vectors were applied via a frictionless cable-pulley system as a function of a vGRF $(0 \%, 25 \%, 50 \%, 75 \%, 100 \%$ vGRF $)$ set at body weight $(534 \mathrm{~N})$. These values were chosen based on physiologic joint loading during stance phase gait, where maximum joint loads reach approximately double BW [37]. AT loads were applied within four degrees of alignment with the vertical tibia axis while the heel was raised $50 \mathrm{~mm}$ and an X-Y table, with a second 6-axis load cell at the base of the foot, was unlocked. This allowed for unconstrained arch formation while properly transferring loads from the tibia to the calcaneus and maintaining foot support throughout the gait simulation $[13,26]$. The heel was then returned to the neutral position and an axial tibia force $\left(\mathrm{F}_{\mathrm{t}}\right)$ of $534 \mathrm{~N}$ was applied to the specimen to meet the desired vGRF condition within a prescribed $\pm 3 \mathrm{~N}$ tolerance. In the passive $(0 \%)$ loading scenario, a force of $5 \mathrm{~N}$ was applied through the tibia instead of the full BW vGRF. After the desired loading condition was met, the $\mathrm{X}-\mathrm{Y}$ table was locked and the rotation of the specimen was started.

A "pure moment" condition was implemented to maintain the axial load through the tibia and minimize off axis loads while rotating about an instantaneous center. The tibia started in a neutral orientation ( 0 degrees) and was rotated about an initial prescribed ankle rotational axis (pARA) (Figure 4-2A) that was measured off of a scaled medial radiograph of the foot processed in Image $(\mathrm{NIH})$. This transformation in the $\mathrm{X}$ and $\mathrm{Z}$ directions from the null tool tip (NTT), located at the center of the gimbal, changed the location of the ankle rotational axis (ARA) to the center of the talus, referred to as the extended tool tip (ETT). Because the tibia was rigidly connected to the RTP, the NTT data remained aligned with the rotated axes of the tibia.

The next step in the test protocol was to establish the kinematic path of the ankle 
A)

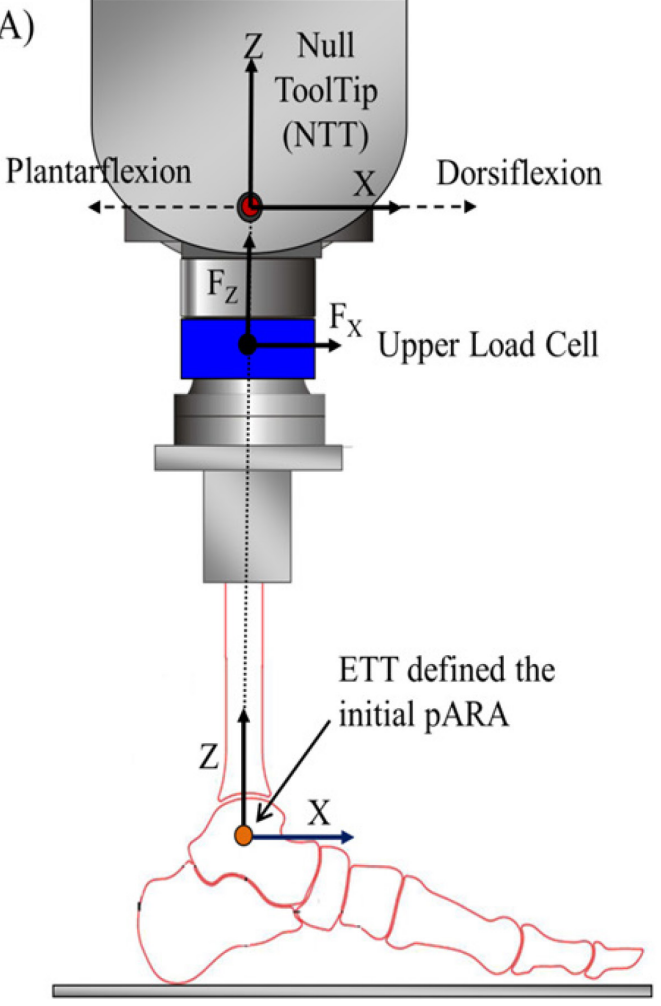

B)

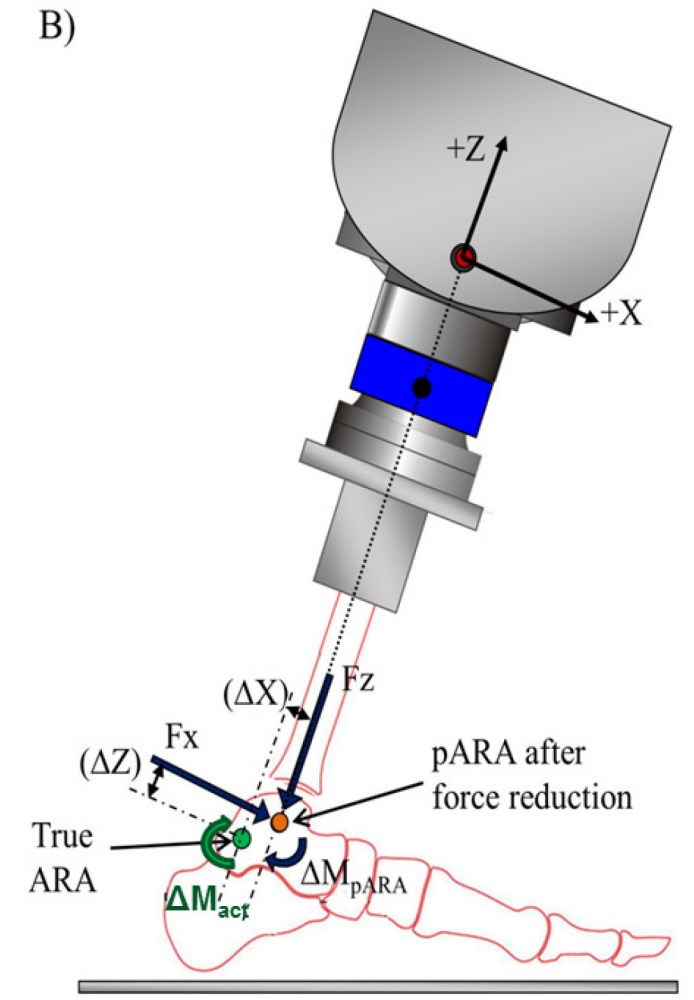

Figure 4-2. Modified "Pure Moment" Program.

Kinematic and load control strategy. A) Kinematic coordinate frames of the foot and ankle are defined by the NTT and ETT of the RTP. The ETT represents the initial prescribed IAR of the foot located at the center of the talus and was a negative $\mathrm{Z}$ offset from the NTT. The coordinate frame of the load cell was initially located at the center of the load cell and transformed to the origin of the moving joint center, i.e., the ETT. B) An angular input of ankle rotation was given every 0.5 degrees, during which the loading protocol reduced the ankle moment due to off-axis forces by minimizing the distance $(\Delta \mathrm{X}, \Delta \mathrm{Z})$ between the pARA (upper) and the true ARA (lower), thus creating a "pure moment" condition. Additional shear and compressive loads could be introduced at this time to maintain the axial tibial force and vGRFs. The NTT data remained aligned with the rotated tibia axes. Achilles load is not shown. 
joint under pure rotational loading. This was accomplished by introducing an incremental rotation to the specimen and then reducing the off-axis forces by minimizing the distance $(\Delta \mathrm{X}, \Delta \mathrm{Z})$ between the pARA and the true ARA (Figure 4-2B), thus a "pure moment" loading condition was achieved. For each incremental rotation, the pure moment loading state could be modified to allow additional shear and compressive forces to be added and directed along the new rotational axes. The process was repeated every 0.5 degrees until the full 10 degrees of dorsiflexion or plantarflexion was achieved. Five cycles were allotted for conditioning of the tissue followed by five cycles. Throughout the testing sequence, specimens were kept moist at regular intervals with a $0.9 \%$ saline mist.

\section{Data Analysis}

Robot positional data and force data were recorded at $20 \mathrm{~Hz}$. A modified version of Reauleux's method [53] for calculating a joint's instantaneous axis of rotation was applied to the robot NTT data to determine the IAR of the ankle within $\pm 0.001 \mathrm{~mm}$. An ankle coordinate frame for describing ankle motion was defined by the bisector of the tibia and highest point on the tibial mortise in a neutral upright orientation. The resultant IAR paths of the ankle motion for each specimen were plotted in Excel.

MMS data were recorded during playback of the the fifth cycle of flexion at $10 \mathrm{~Hz}$. Target translation data were transformed to K-wire tips implanted in the bone. XYZ Euler decomposition was used to subtract the effect of rotation on the translational data and transform the rotated reference frame of the k-wires to the sagittal plane (Appendix B) [59]. Bones were considered at neutral position and rotation when the tibia was in the upright position perpendicular to the ground in the sagittal plane and the vGRF was at maximum force. Marker positions were determined accurately within \pm 80 microns.

Mean IAR values, mean bone rotations, and mean bone translations were compared between loading conditions and between dorsiflexion and plantarflexion for each specimen using a One Way ANOVA with a Holm Sidak Post Hoc Multiple Comparisons test. Results are presented as means \pm standard deviations. A P-value of less than 0.05 signified significant differences between comparisons.

\section{Results}

IAR paths throughout motion are displayed in Figure 4-3. Paths for specimen 1 and 2 showed little movement in the talus for both dorsiflexion and plantarflexion, remaining within a $7 \mathrm{~mm}$ range (i.e., a maximum of $3.5 \mathrm{~mm}$ in the $\mathrm{X}$ and $6 \mathrm{~mm}$ in the $\mathrm{Z}$ ). Specimen 3 and 4 showed more movement throughout the talus, remaining within a $15 \mathrm{~mm}$ range (i.e., a maximum of $4 \mathrm{~mm}$ in the $X$ and $20 \mathrm{~mm}$ in the $Z$ ). In specimen 3 , the $0 \%$ Achilles load paths showed the most movement, whereas in the other 3 specimens the $0 \%$ Achilles load generally had little movement, demonstrating a possible fixed point 
Figure 4-3. IAR Paths.

(A) Specimen 1, (B) Specimen 2, (C) Specimen 3, (D) Specimen 4. Percentages refer to the amount of Achilles load in reference to the vGRF. Arrows denote the direction of the path throughout 10 degrees dorsiflexion or plantarflexion. 

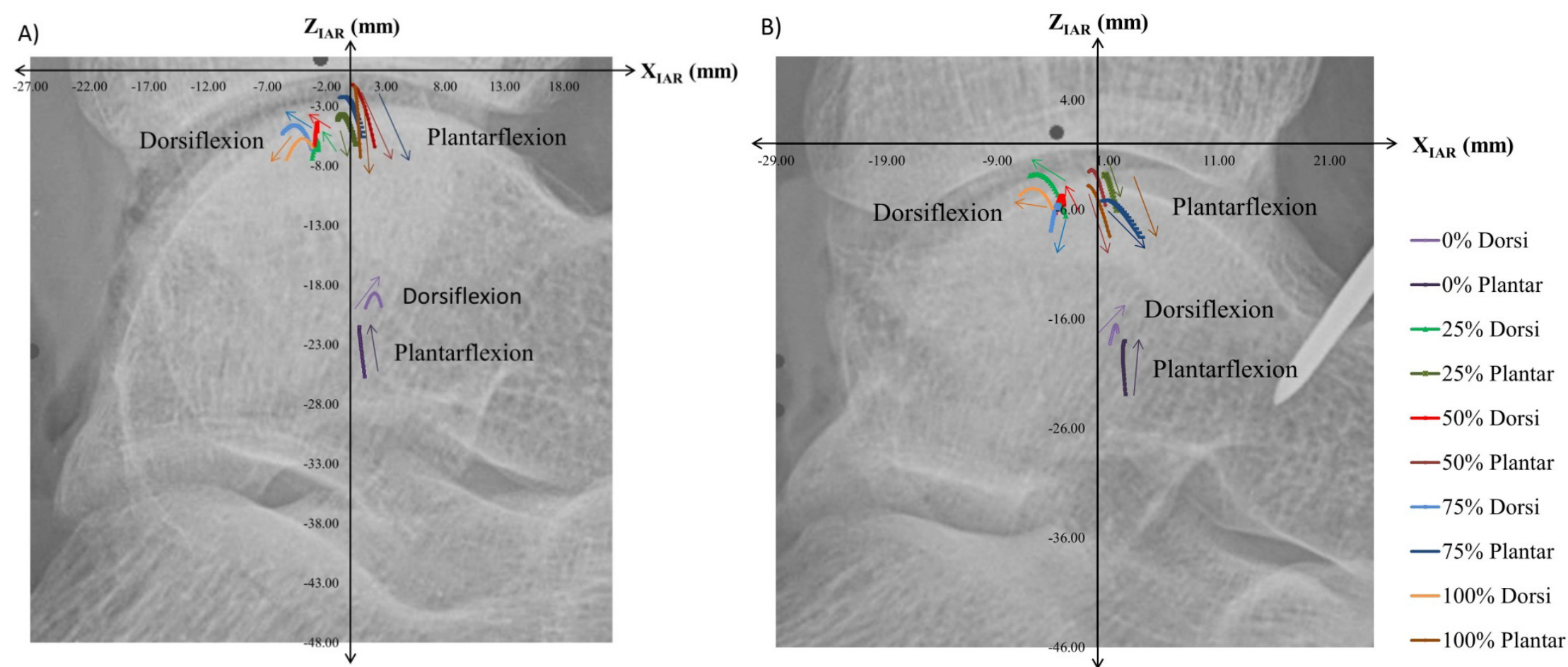

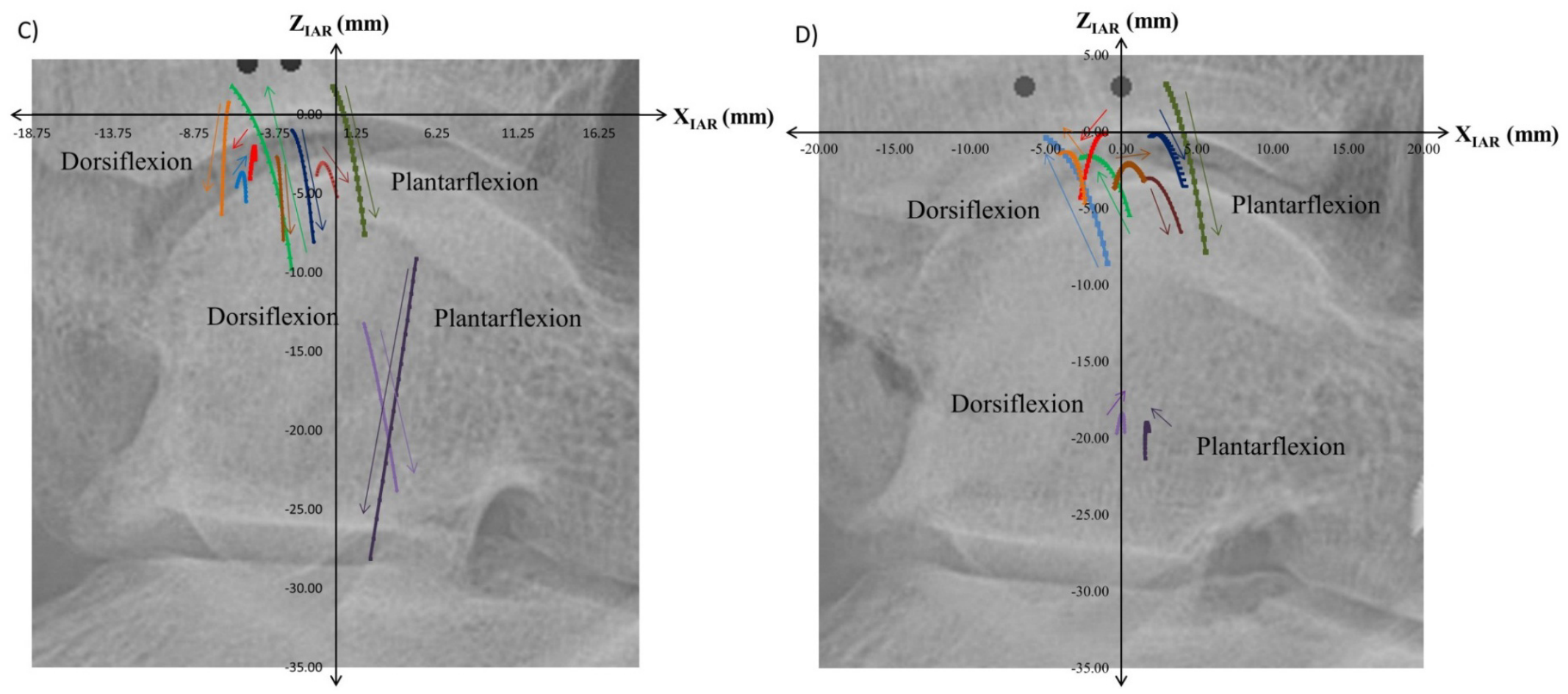

Figure 4-3. Continued. 
rotation where the tibial mortise motion follows the surface of the talar dome. With a loaded Achilles, all paths started up towards the articular surfaces for each specimen. Dorsiflexion paths with an active Achilles load in specimen 1, 2, and 4 consistently travelled posteriorly throughout motion. In contrast, specimen 3 paths changed directions between loading conditions with no apparent pattern. However, the paths that travelled anteriorly remained within a tight range, demonstrating a possible fixed point rotation. In plantarflexion for all specimens with a loaded Achilles, the first degrees of motion started up within the articular surface of the talus. As rotation continued, the IAR path moved downward towards the middle of the talus, transitioning from a fixed point rotation at the joint articular surfaces to a sliding motion between them. Under these loading conditions, the IAR appeared to move closer to the rotational center as defined by the arc of the tibiotalar articulating surfaces.

Mean IAR values for all specimens are shown in Table 4-1 through Table 4-4, respectively. Significant differences in mean XIAR values between dorsi- and plantarflexion of the same loading condition were found in all cases for all specimens: dorsiflexion mean values were posterior to the plantarflexion mean XIAR values. Significant differences in mean ZIAR values between dorsi- and plantar-flexion of the same loading condition were found in all cases for specimens 1 and 2 and in the 0 and 50 percent loaded conditions in specimen 4. XIAR values in dorsiflexion generally showed a significant difference between loading conditions with a posterior shift due to an increased Achilles load. Plantarflexion mean XIAR values did not show this same pattern. While in the majority of cases there were significant differences between mean XIAR values between loaded conditions in plantarflexion, values did not show an apparent posterior or anterior shift due to increasing Achilles load. Mean ZIAR values in dorsiflexion and plantarflexion demonstrated a significant upward shift due to a minimal Achilles load (between $10 \mathrm{~mm}$ and $20 \mathrm{~mm}$ ). Differences were found in the majority of cases of mean ZIAR values between the active loaded Achilles conditions. However, there does not seem to be an apparent pattern to suggest a cause other than each path will incorporate variable amounts of rolling and sliding between the articular surfaces. Once the Achilles was loaded, mean XIAR and ZIAR values remained within 3mm (maximum shift was $5.5 \mathrm{~mm}$ in specimen 3 ). These results suggest a minimal AT load is sufficient in actively loading the arches of the foot. They also suggest net joint loading had no effect on the IAR of the foot-ankle complex once the Achilles was loaded (net joint load: 667.5$1068 \mathrm{~N})$.

Mean rotations and translations for the talus, calcaneus, and navicular for specimens 1-4 are shown in Table 4-5 through Table 4-8, respectively. Data was kept separate for each specimen to demonstrate interspecimen variability. Mean data for all four specimens is shown in Appendix C. Positive rotations in the X, Y, and Z axes corresponded to eversion, adduction, and dorsiflexion, respectively for a right foot (specimen 2 and 4). Positive $\mathrm{X}$ and $\mathrm{Y}$ rotations were opposite for left feet such that positive rotations correlated to inversion and abduction for specimens 1 and 3 . Positive translations in the $\mathrm{X}, \mathrm{Y}$, and $\mathrm{Z}$ axes corresponded to anterior, superior, and lateral translations, respectively for a right foot (specimen 2 and 4). Positive $Z$ translations were opposite for left feet such that a positive value in specimens 1 and 3 correlated to medial 
Table 4-1. Mean IAR Values between Loading Conditions for Specimen 1.

\begin{tabular}{|c|c|c|c|c|}
\hline \multirow{2}{*}{$\begin{array}{c}\% \\
\text { vGRF } \\
\text { AT } \\
\text { Load }\end{array}$} & \multicolumn{2}{|c|}{$\begin{aligned} & \text { Mean XIAR }(\mathrm{mm}) \\
\pm & \text { Standard Deviations }\end{aligned}$} & \multicolumn{2}{|c|}{$\begin{aligned} & \text { Mean ZIAR }(\mathrm{mm}) \\
\pm & \text { Standard Deviations }\end{aligned}$} \\
\hline & Dorsiflexion & Plantarflexion & Dorsiflexion & Plantarflexion \\
\hline 0 & $1.972 \pm 0.418^{\# \sim}$ & $0.849 \pm 0.152^{\#^{\prime}}$ & $-20.280 \pm 0.400^{\#-}$ & $-24.000 \pm 1.358^{\#}$ \\
\hline 25 & $-2.917 \pm 0.162^{*^{\wedge}+}$ & $-0.378 \pm 0.466^{*^{\sim+}}$ & $-7.402 \pm 0.453^{*}$ & $-5.536 \pm 0.763^{*^{\sim}}$ \\
\hline 50 & $-2.867 \pm 0.075^{*^{\wedge+}}$ & $1.033 \pm 0.597^{\#+}$ & $-6.084 \pm 0.529^{* \#+}$ & $-4.180 \pm 1.663^{* \#}$ \\
\hline 75 & $-4.439 \pm 0.756^{* \# \sim ~}$ & $-0.0680 \pm 0.596^{*^{\sim+}}$ & $-6.310 \pm 0.582^{* \#}$ & $-4.330 \pm 1.099^{* \#}$ \\
\hline 100 & $-4.262 \pm 0.675^{* \# \sim}$ & $0.5745 \pm 0.144^{\#}$ & $-7.368 \pm 0.516^{* \sim}$ & $-4.676 \pm 1.892^{*}$ \\
\hline
\end{tabular}

${ }^{*}$ Significant difference with $0 \%$

\# Significant difference with $25 \%$

Significant difference with $50 \%$

Significant difference with $75 \%$

+ Significant difference with $100 \%$

Note: Significant differences between dorsi- and plantar-flexion were found in all cases in mean XIAR and ZIAR values of the same loading condition.

Table 4-2. Mean IAR Values between Loading Conditions for Specimen 2.

\begin{tabular}{|c|c|c|c|c|}
\hline \multirow{2}{*}{$\begin{array}{c}\% \\
\text { vGRF } \\
\text { AT } \\
\text { Load }\end{array}$} & \multicolumn{2}{|c|}{$\begin{aligned} & \text { Mean XIAR }(\mathrm{mm}) \\
\pm & \text { Standard Deviations }\end{aligned}$} & \multicolumn{2}{|c|}{$\begin{aligned} & \text { Mean ZIAR (mm) } \\
\pm & \text { Standard Deviations }\end{aligned}$} \\
\hline & Dorsiflexion & Plantarflexion & Dorsiflexion & Plantarflexion \\
\hline 0 & $1.573 \pm 0.224^{\# \sim^{\wedge}+}$ & $2.386 \pm 0.075^{\# \sim^{\wedge}+}$ & $-17.022 \pm 0.520^{\# \sim^{\wedge+}}$ & $-19.691 \pm 1.568^{\# \sim \wedge+}$ \\
\hline 25 & $-4.351 \pm 1.008^{*^{\wedge+}}$ & $1.068 \pm 0.351^{*^{\wedge}+}$ & $-3.921 \pm 1.246^{*^{\wedge}}$ & $-3.751 \pm 1.062^{*^{\wedge+}}$ \\
\hline 50 & $-3.297 \pm 0.243^{* \#+}$ & $-0.069 \pm 0.466^{* \#^{\wedge}}$ & $-5.191 \pm 0.454^{* \#^{\wedge}}$ & $-3.275 \pm 1.032^{* \#^{\wedge}+}$ \\
\hline 75 & $-3.748 \pm 0.232^{* \#+}$ & $1.823 \pm 1.090^{* \# \sim}$ & $-6.201 \pm 0.724^{* \# \sim+}$ & $-6.119 \pm 1.099^{* \# \sim ~}$ \\
\hline 100 & $-5.419 \pm 0.914^{* \# \sim \wedge}$ & $0.063 \pm 0.614^{* \#}$ & $-4.576 \pm 0.547^{*^{\wedge}}$ & $-5.388 \pm 1.496^{* \# \sim}$ \\
\hline
\end{tabular}

${ }^{*}$ Significant difference with $0 \%$

\# Significant difference with $25 \%$

Significant difference with 50\%

Significant difference with $75 \%$

+ Significant difference with $100 \%$

Note: Significant differences between dorsi- and plantar-flexion were found in all cases between mean XIAR and between 0 and 50 loading conditions in ZIAR values of the same loading condition. 
Table 4-3. Mean IAR Values between Loading Conditions for Specimen 3.

\begin{tabular}{|c|c|c|c|c|}
\hline \multirow{2}{*}{$\begin{array}{c}\% \\
\text { vGRF } \\
\text { AT } \\
\text { Load }\end{array}$} & \multicolumn{2}{|c|}{$\begin{array}{l}\text { Mean XIAR (mm) } \\
\pm \text { Standard Deviations }\end{array}$} & \multicolumn{2}{|c|}{$\begin{array}{c}\text { Mean ZIAR }(\mathrm{mm}) \\
\pm \text { Standard Deviations }\end{array}$} \\
\hline & Dorsiflexion & Plantarflexion & Dorsiflexion & Plantarflexion \\
\hline 0 & $2.668 \pm 0.641^{\#-}$ & $3.701 \pm 0.890^{\text {\# }}$ & $-17.658 \pm 3.334^{\#-}$ & $-17.756 \pm 5.950^{\# \wedge \wedge+}$ \\
\hline 25 & $-4.339 \pm 1.157^{*^{\wedge+}}$ & $0.831 \pm 0.615^{*^{\sim+}}$ & $-3.128 \pm 3.655^{*}$ & $-2.042 \pm 2.948^{*}$ \\
\hline 50 & $-5.086 \pm 0.102^{* *^{A+}}$ & $-0.625 \pm 0.394^{* \#^{\wedge}+}$ & $-2.618 \pm 0.582^{*}$ & $-3.646 \pm 0.626^{*}$ \\
\hline 75 & $-5.779 \pm 0.185^{* * \sim+}$ & $-2.034 \pm 0.418^{* \# \sim+}$ & $-4.203 \pm 0.531^{*^{+}}$ & $-3.654 \pm 2.249^{*}$ \\
\hline 100 & $-6.845 \pm 0.142^{* \# \wedge \wedge}$ & $3.453 \pm 0.135^{* \# \sim 1}$ & $-1.930 \pm 2.280^{*^{\wedge}}$ & $-4.465 \pm 1.696^{*}$ \\
\hline
\end{tabular}

* Significant difference with $0 \%$

${ }^{\text {\# }}$ Significant difference with $25 \%$

$\sim$ Significant difference with $50 \%$

${ }^{\wedge}$ Significant difference with $75 \%$

${ }^{+}$Significant difference with $100 \%$

Note: Significant differences between dorsi- and plantar-flexion were found in all cases between mean XIAR, but no significant differences were found in ZIAR.

\section{Table 4-4. Mean IAR Values between Loading Conditions for Specimen 4.}

\begin{tabular}{|c|c|c|c|c|}
\hline \multirow{2}{*}{$\begin{array}{c}\% \\
\text { vGRF } \\
\text { AT } \\
\text { Load }\end{array}$} & \multicolumn{2}{|c|}{$\begin{aligned} & \text { Mean XIAR }(\mathrm{mm}) \\
\pm & \text { Standard Deviations }\end{aligned}$} & \multicolumn{2}{|c|}{$\begin{array}{c}\text { Mean ZIAR }(\mathrm{mm}) \\
\pm \text { Standard Deviations }\end{array}$} \\
\hline & Dorsiflexion & Plantarflexion & Dorsiflexion & Plantarflexion \\
\hline 0 & $0.012 \pm 0.164^{\#-}$ & $1.639 \pm 0.090^{\# \sim}$ & $-18.847 \pm 0.434^{\#}$ & $-19.604 \pm 0.706^{\#}$ \\
\hline 25 & $-0.971 \pm 1.008^{*^{\wedge+}}$ & $4.310 \pm 0.818^{*^{\wedge+}}$ & $-2.721 \pm 1.246^{*}$ & $-1.542 \pm 3.438^{* \sim}$ \\
\hline 50 & $-1.854 \pm 0.535^{*^{\#+}}$ & $2.627 \pm 0.784^{* \#+}$ & $-1.416 \pm 1.374^{*^{*+}}$ & $-4.039 \pm 1.127^{* *^{\wedge}}$ \\
\hline 75 & $-2.766 \pm 1.273^{* \# \sim t}$ & $2.888 \pm 0.698^{* *+}$ & $-3.688 \pm 2.595^{* \sim}$ & $-1.136 \pm 1.098^{* \sim}$ \\
\hline 100 & $-3.122 \pm 0.534^{* \# \sim}$ & $0.443 \pm 0.614^{* \# \sim \wedge}$ & $-2.297 \pm 1.105^{* \sim}$ & $-2.593 \pm 0.458^{*}$ \\
\hline
\end{tabular}

* Significant difference with $0 \%$

${ }^{\#}$ Significant difference with $25 \%$

$\sim$ Significant difference with $50 \%$

${ }^{\wedge}$ Significant difference with $75 \%$

${ }^{+}$Significant difference with $100 \%$

Note: Significant differences between dorsi- and plantar-flexion were found in all cases between mean XIAR and in the 50 and 75 loading conditions between ZIAR. 
Table 4-5. Specimen 1 Translations and Rotations.

\begin{tabular}{|c|c|c|c|c|c|c|c|c|c|}
\hline \multirow{4}{*}{$\begin{array}{c}\% \\
\text { vGRF } \\
\text { AT } \\
\text { Load }\end{array}$} & \multicolumn{9}{|c|}{ Translation $(\mathrm{mm}) \pm$ Standard Deviation } \\
\hline & \multicolumn{9}{|c|}{ Dorsiflexion } \\
\hline & \multicolumn{3}{|c|}{ Calcaneus } & \multicolumn{3}{|c|}{ Talus } & \multicolumn{3}{|c|}{ Navicular } \\
\hline & $\mathrm{X}$ & $\mathrm{Y}$ & $\mathrm{Z}$ & $\mathrm{X}$ & $\mathrm{Y}$ & Z & $\mathrm{X}$ & $\mathrm{Y}$ & Z \\
\hline 0 & $-1.299 \pm 0.104$ & $-0.156 \pm 0.386$ & $0.968 \pm 0.061$ & $-0.438 \pm 0.970$ & $0.373 \pm 0.172$ & $3.458 \pm 0.386$ & $0.598 \pm 0.359$ & $-0.438 \pm 0.173$ & $2.747 \pm 0.215$ \\
\hline 25 & $-4.312 \pm 0.276$ & $-0.467 \pm 0.657$ & $3.977 \pm 0.298$ & $-3.004 \pm 0.487$ & $1.012 \pm 0.347$ & $1.037 \pm 0.117$ & $-0.806 \pm 0.562$ & $-1.219 \pm 0.061$ & $-1.432 \pm 0.545$ \\
\hline 50 & $-5.439 \pm 1.849$ & $0.529 \pm 0.692$ & $2.377 \pm 0.042$ & $-3.281 \pm 0.993$ & $-0.184 \pm 0.763$ & $1.916 \pm 0.607$ & $-3.818 \pm 0.606$ & $-2.987 \pm 0.038$ & $-6.219 \pm 0.831$ \\
\hline 75 & $-4.165 \pm 0.916$ & $-0.730 \pm 0.088$ & $0.589 \pm 0.299$ & $-3.905 \pm 0.214$ & $-0.135 \pm 0.283$ & $4.272 \pm 0.710$ & $-0.197 \pm 0.087$ & $-3.216 \pm 1.145$ & $-2.524 \pm 0.691$ \\
\hline \multirow[t]{2}{*}{100} & $-3.232 \pm 0.633$ & $-0.567 \pm 0.175$ & $2.492 \pm 0.719$ & $2.525 \pm 0.220$ & $-0.259 \pm 0.535$ & $1.437 \pm 0.344$ & $-0.693 \pm 0.381$ & $-2.099 \pm 0.204$ & $-5.580 \pm 0.971$ \\
\hline & \multicolumn{9}{|c|}{ Plantarflexion } \\
\hline 0 & $0.950 \pm 0.282$ & $0.211 \pm 0.498$ & $-1.652 \pm 0.700$ & $1.166 \pm 0.178$ & $0.712 \pm 0.746$ & $-2.796 \pm 0.766$ & $-0.216 \pm 0.470$ & $-1.912 \pm 0.097$ & $-2.282 \pm 0.925$ \\
\hline 25 & $1.605 \pm 1.537$ & $-0.505 \pm 0.106$ & $-3.114 \pm 0.761$ & $2.165 \pm 0.910$ & $1.575 \pm 0.231$ & $-3.019 \pm 0.590$ & $0.659 \pm 1.500$ & $-0.400 \pm 0.186$ & $1.281 \pm 0.338$ \\
\hline 50 & $2.523 \pm 0.145$ & $0.038 \pm 0.968$ & $-1.810 \pm 0.057$ & $3.354 \pm 0.162$ & $0.344 \pm 0.068$ & $-1.182 \pm 0.651$ & $-0.468 \pm 0.039$ & $-1.225 \pm 0.278$ & $-2.596 \pm 0.409$ \\
\hline 75 & $3.663 \pm 0.233$ & $1.185 \pm 0.115$ & $4.223 \pm 1.252$ & $1.333 \pm 0.550$ & $-1.511 \pm 0.697$ & $1.112 \pm 0.956$ & $2.765 \pm 0.311$ & $-0.851 \pm 0.326$ & $-3.028 \pm 1.069$ \\
\hline 100 & $3.898 \pm 0.945$ & $0.640 \pm 0.385$ & $-4.088 \pm 0.675$ & $4.667 \pm 0.914$ & $-0.315 \pm 0.146$ & $-1.770 \pm 0.853$ & $0.249 \pm 0.110$ & $-1.730 \pm 0.198$ & $7.042 \pm 1.113$ \\
\hline$\%$ & \multicolumn{9}{|c|}{ Rotation (degrees) \pm Standard Deviation } \\
\hline vGRF & \multicolumn{9}{|c|}{ Dorsiflexion } \\
\hline AT & \multicolumn{3}{|c|}{ Calcaneus } & \multicolumn{3}{|c|}{ Talus } & \multicolumn{3}{|c|}{ Navicular } \\
\hline Load & $\mathrm{X}$ & $\mathrm{Y}$ & $\mathrm{Z}$ & $\mathrm{X}$ & $\mathrm{Y}$ & $\mathrm{Z}$ & $\mathrm{X}$ & $\mathrm{Y}$ & $\mathrm{Z}$ \\
\hline 0 & $-0.136 \pm 0.284$ & $1.355 \pm 0.054$ & $0.667 \pm 0.106$ & $-0.373 \pm 0.633$ & $0.855 \pm 0.599$ & $0.945 \pm 0.344$ & $0.344 \pm 0.320$ & $-0.402 \pm 0.051$ & $0.404 \pm 0.395$ \\
\hline 25 & $-3.125 \pm 0.281$ & $-0.368 \pm 0.498$ & $0.658 \pm 0.160$ & $0.943 \pm 0.107$ & $2.651 \pm 0.285$ & $0.801 \pm 0.133$ & $1.914 \pm 0.170$ & $0.169 \pm 0.041$ & $0.124 \pm 0.010$ \\
\hline 50 & $0.587 \pm 0.319$ & $1.318 \pm 0.395$ & $0.919 \pm 0.366$ & $2.535 \pm 0.045$ & $-0.844 \pm 0.157$ & $0.439 \pm 0.373$ & $3.865 \pm 0.353$ & $0.046 \pm 0.156$ & $0.165 \pm 0.149$ \\
\hline 75 & $2.655 \pm 0.700$ & $0.335 \pm 0.357$ & $0.667 \pm 0.019$ & $1.934 \pm 0.124$ & $0.982 \pm 0.146$ & $-0.465 \pm 0.022$ & $1.325 \pm 0.928$ & $0.567 \pm 0.143$ & $0.165 \pm 0.043$ \\
\hline \multirow[t]{2}{*}{100} & $0.354 \pm 0.157$ & $0.016 \pm 0.018$ & $0.476 \pm 0.184$ & $1.186 \pm 0.102$ & $0.333 \pm 0.076$ & $1.000 \pm 0.262$ & $2.148 \pm 0.523$ & $0.015 \pm 0.002$ & $0.497 \pm 0.134$ \\
\hline & \multicolumn{9}{|c|}{ Plantarflexion } \\
\hline 0 & $-1.667 \pm 0.021$ & $-1.015 \pm 0.394$ & $-0.947 \pm 0.379$ & $-0.168 \pm 0.401$ & $-3.667 \pm 0.665$ & $-0.947 \pm 0.435$ & $-1.000 \pm 0.852$ & $-0.622 \pm 0.557$ & $0.205 \pm 0.011$ \\
\hline 25 & $0.318 \pm 0.460$ & $-2.107 \pm 0.334$ & $0.466 \pm 0.133$ & $-0.879 \pm 0.292$ & $-1.946 \pm 0.354$ & $-0.702 \pm 0.144$ & $-0.356 \pm 0.584$ & $-2.259 \pm 0.634$ & $-0.255 \pm 0.199$ \\
\hline 50 & $3.165 \pm 0.216$ & $2.255 \pm 0.416$ & $0.347 \pm 0.579$ & $0.355 \pm 0.159$ & $-2.469 \pm 0.175$ & $-0.648 \pm 0.171$ & $2.345 \pm 0.024$ & $0.355 \pm 0.087$ & $0.247 \pm 0.195$ \\
\hline 75 & $-0.347 \pm 0.157$ & $1.547 \pm 0.448$ & $0.484 \pm 0.013$ & $1.789 \pm 0.169$ & $-0.716 \pm 0.096$ & $-0.457 \pm 0.650$ & $-0.942 \pm 0.149$ & $-1.355 \pm 0.311$ & $-0.333 \pm 0.219$ \\
\hline 100 & $2.366 \pm 0.420$ & $0.971 \pm 0.206$ & $0.655 \pm 0.039$ & $0.197 \pm 0.084$ & $-0.844 \pm 0.047$ & $-0.328 \pm 0.138$ & $-0.945 \pm 0.383$ & $0.215 \pm 0.133$ & $-0.427 \pm 0.297$ \\
\hline
\end{tabular}

Positive rotations in the $\mathrm{X}, \mathrm{Y}$, and $\mathrm{Z}$ axes corresponded to inversion, abduction, and plantarflexion. Positive translations in the $\mathrm{X}$, $\mathrm{Y}$, and $\mathrm{Z}$ axes corresponded to anterior, superior, and medial translations. 
Table 4-6. Specimen 2 Translations and Rotations.

\begin{tabular}{|c|c|c|c|c|c|c|c|c|c|}
\hline \multirow{4}{*}{$\begin{array}{c}\% \\
\text { vGRF } \\
\text { AT } \\
\text { Load }\end{array}$} & \multicolumn{9}{|c|}{ Translation $(\mathrm{mm}) \pm$ Standard Deviation } \\
\hline & \multicolumn{9}{|c|}{ Dorsiflexion } \\
\hline & \multicolumn{3}{|c|}{ Calcaneus } & \multicolumn{3}{|c|}{ Talus } & \multicolumn{3}{|c|}{ Navicular } \\
\hline & $\mathrm{X}$ & $\mathrm{Y}$ & Z & $\mathrm{X}$ & $\mathrm{Y}$ & $\mathrm{Z}$ & $\mathrm{X}$ & $\mathrm{Y}$ & Z \\
\hline 0 & $1.130 \pm 0.164$ & $0.366 \pm 0.195$ & $-1.617 \pm 0.562$ & $-1.084 \pm 0.397$ & $0.413 \pm 0.077$ & $0.646 \pm 0.286$ & $-1.272 \pm 0.076$ & $-0.526 \pm 0.289$ & $3.212 \pm 0.495$ \\
\hline 25 & $-3.558 \pm 0.726$ & $2.047 \pm 1.566$ & $0.530 \pm 0.898$ & $-2.190 \pm 0.788$ & $0.994 \pm 0.286$ & $1.904 \pm 0.412$ & $2.943 \pm 0.056$ & $-1.245 \pm 0.162$ & $3.024 \pm 0.764$ \\
\hline 50 & $-4.696 \pm 0.493$ & $0.503 \pm 0.304$ & $-0.009 \pm 0.048$ & $-1.963 \pm 0.193$ & $-0.402 \pm 0.633$ & $-1.162 \pm 0.061$ & $4.222 \pm 0.561$ & $-1.901 \pm 0.290$ & $3.413 \pm 0.306$ \\
\hline 75 & $-3.742 \pm 0.601$ & $0.837 \pm 0.788$ & $0.421 \pm 0.099$ & $-4.077 \pm 0.138$ & $0.008 \pm 0.832$ & $2.178 \pm 1.102$ & $-0.974 \pm 1.409$ & $-1.216 \pm 0.452$ & $1.244 \pm 0.610$ \\
\hline \multirow[t]{2}{*}{100} & $-4.817 \pm 0.365$ & $-2.614 \pm 0.184$ & $5.595 \pm 0.979$ & $3.415 \pm 0.513$ & $-0.103 \pm 0.437$ & $-1.679 \pm 0.288$ & $-1.078 \pm 0.298$ & $-0.697 \pm 0.987$ & $4.758 \pm 0.713$ \\
\hline & \multicolumn{9}{|c|}{ Plantarflexion } \\
\hline 0 & $0.420 \pm 0.150$ & $0.120 \pm 0.098$ & $1.752 \pm 0.270$ & $-0.667 \pm 0.018$ & $0.858 \pm 0.464$ & $1.790 \pm 0.277$ & $1.157 \pm 0.099$ & $-0.912 \pm 0.097$ & $-2.869 \pm 0.764$ \\
\hline 25 & $-0.545 \pm 0.625$ & $0.536 \pm 0.461$ & $2.143 \pm 0.904$ & $2.667 \pm 0.491$ & $-0.575 \pm 0.021$ & $3.991 \pm 0.526$ & $-0.405 \pm 0.186$ & $-0.585 \pm 0.282$ & $-5.061 \pm 0.376$ \\
\hline 50 & $2.231 \pm 0.261$ & $1.031 \pm 0.097$ & $2.860 \pm 0.081$ & $3.666 \pm 0.624$ & $-1.548 \pm 0.478$ & $6.018 \pm 0.635$ & $-2.438 \pm 0.393$ & $-0.252 \pm 0.082$ & $-2.956 \pm 0.394$ \\
\hline 75 & $3.663 \pm 0.233$ & $1.185 \pm 0.115$ & $4.223 \pm 1.252$ & $1.333 \pm 0.550$ & $-1.511 \pm 0.697$ & $1.112 \pm 0.956$ & $2.765 \pm 0.311$ & $-0.851 \pm 0.326$ & $-3.028 \pm 1.069$ \\
\hline 100 & $2.390 \pm 0.168$ & $1.640 \pm 0.048$ & $3.009 \pm 0.267$ & $1.727 \pm 0.136$ & $-0.823 \pm 0.145$ & $1.770 \pm 0.435$ & $2.005 \pm 1.190$ & $-1.639 \pm 0.222$ & $-1.904 \pm 1.039$ \\
\hline$\%$ & \multicolumn{9}{|c|}{ Rotation (degrees) \pm Standard Deviation } \\
\hline vGRF & \multicolumn{9}{|c|}{ Dorsiflexion } \\
\hline $\mathrm{AT}$ & \multicolumn{3}{|c|}{ Calcaneus } & \multicolumn{3}{|c|}{ Talus } & \multicolumn{3}{|c|}{ Navicular } \\
\hline Load & $\mathrm{X}$ & $\mathrm{Y}$ & Z & $\mathrm{X}$ & $\mathrm{Y}$ & $\mathrm{Z}$ & $\mathrm{X}$ & $\mathrm{Y}$ & $\mathrm{Z}$ \\
\hline 0 & $1.069 \pm 0.184$ & $0.769 \pm 0.504$ & $0.508 \pm 0.161$ & $-1.333 \pm 0.332$ & $0.233 \pm 0.079$ & $-1.000 \pm 0.093$ & $0.667 \pm 0.199$ & $-0.219 \pm 0.438$ & $0.026 \pm 0.542$ \\
\hline 25 & $2.647 \pm 0.740$ & $0.659 \pm 0.101$ & $0.333 \pm 0.603$ & $-1.680 \pm 0.031$ & $0.567 \pm 0.085$ & $0.168 \pm 0.093$ & $-1.369 \pm 0.118$ & $-0.368 \pm 0.415$ & $0.333 \pm 0.198$ \\
\hline 50 & $0.857 \pm 0.174$ & $-0.265 \pm 0.140$ & $-1.000 \pm 0.366$ & $0.789 \pm 0.055$ & $0.915 \pm 0.573$ & $0.686 \pm 0.043$ & $0.818 \pm 0.703$ & $-1.005 \pm 0.042$ & $1.649 \pm 0.942$ \\
\hline 75 & $-0.467 \pm 0.003$ & $0.667 \pm 0.178$ & $-0.665 \pm 0.865$ & $-1.265 \pm 0.227$ & $1.667 \pm 0.190$ & $-0.169 \pm 0.602$ & $2.123 \pm 0.042$ & $0.126 \pm 0.714$ & $-0.333 \pm 0.346$ \\
\hline \multirow[t]{2}{*}{100} & $2.106 \pm 0.309$ & $0.267 \pm 0.154$ & $0.292 \pm 0.134$ & $1.386 \pm 0.870$ & $0.876 \pm 0.275$ & $0.049 \pm 0.311$ & $-0.946 \pm 0.152$ & $1.069 \pm 0.002$ & $-0.147 \pm 0.734$ \\
\hline & \multicolumn{9}{|c|}{ Plantarflexion } \\
\hline 0 & $-0.408 \pm 0.021$ & $-0.637 \pm 0.479$ & $0.794 \pm 0.379$ & $-0.333 \pm 0.401$ & $-2.580 \pm 0.267$ & $1.265 \pm 0.435$ & $0.359 \pm 0.515$ & $0.667 \pm 0.565$ & $-1.000 \pm 0.246$ \\
\hline 25 & $-1.646 \pm 0.596$ & $-0.649 \pm 0.443$ & $1.460 \pm 0.343$ & $3.632 \pm 0.393$ & $-0.669 \pm 0.435$ & $-0.333 \pm 0.474$ & $1.247 \pm 0.058$ & $2.767 \pm 0.344$ & $-0.686 \pm 0.392$ \\
\hline 50 & $-1.315 \pm 0.355$ & $0.957 \pm 0.472$ & $0.892 \pm 0.579$ & $0.107 \pm 0.586$ & $2.127 \pm 0.849$ & $-0.690 \pm 0.371$ & $1.376 \pm 0.291$ & $-0.165 \pm 0.071$ & $-0.700 \pm 0.295$ \\
\hline 75 & $-1.667 \pm 0.663$ & $-0.270 \pm 0.483$ & $0.548 \pm 0.193$ & $0.943 \pm 0.459$ & $0.971 \pm 0.964$ & $0.588 \pm 0.503$ & $0.949 \pm 0.100$ & $0.845 \pm 0.660$ & $-0.914 \pm 0.245$ \\
\hline 100 & $-2.155 \pm 0.144$ & $1.333 \pm 0.192$ & $1.156 \pm 0.290$ & $0.467 \pm 0.484$ & $0.921 \pm 0.247$ & $0.333 \pm 0.161$ & $-0.174 \pm 0.738$ & $-1.832 \pm 0.733$ & $-0.667 \pm 0.968$ \\
\hline
\end{tabular}


Table 4-7. Specimen 3 Translations and Rotations.

\begin{tabular}{|c|c|c|c|c|c|c|c|c|c|}
\hline \multirow{4}{*}{$\begin{array}{c}\% \\
\text { vGRF } \\
\text { AT } \\
\text { Load }\end{array}$} & \multicolumn{9}{|c|}{ Translation $(\mathrm{mm}) \pm$ Standard Deviation } \\
\hline & \multicolumn{9}{|c|}{ Dorsiflexion } \\
\hline & \multicolumn{3}{|c|}{ Calcaneus } & \multicolumn{3}{|c|}{ Talus } & \multicolumn{3}{|c|}{ Navicular } \\
\hline & $\mathrm{X}$ & $\mathrm{Y}$ & $\mathrm{Z}$ & $\mathrm{X}$ & $\mathrm{Y}$ & $\mathrm{Z}$ & $\mathrm{X}$ & $\mathrm{Y}$ & $\mathrm{Z}$ \\
\hline 0 & $3.086 \pm 0.248$ & $-0.136 \pm 1.633$ & $-0.361 \pm 1.160$ & $-0.091 \pm 0.609$ & $0.301 \pm 0.450$ & $2.233 \pm 0.926$ & $0.417 \pm 0.660$ & $0.874 \pm 0.170$ & $-2.317 \pm 0.395$ \\
\hline 25 & $-2.775 \pm 0.374$ & $-4.442 \pm 1.742$ & $6.805 \pm 1.911$ & $5.174 \pm 0.128$ & $0.096 \pm 0.086$ & $2.585 \pm 1.372$ & $-1.661 \pm 1.343$ & $1.383 \pm 1.318$ & $-4.705 \pm 0.364$ \\
\hline 50 & $-4.531 \pm 1.784$ & $1.560 \pm 0.634$ & $0.071 \pm 0.903$ & $6.100 \pm 1.341$ & $-1.874 \pm 1.055$ & $2.338 \pm 1.223$ & $-1.247 \pm 0.225$ & $3.151 \pm 0.075$ & $-10.918 \pm 0.581$ \\
\hline 75 & $-1.638 \pm 0.610$ & $-0.001 \pm 1.067$ & $0.652 \pm 0.987$ & $3.916 \pm 0.587$ & $1.174 \pm 0.073$ & $2.597 \pm 2.085$ & $-0.702 \pm 1.936$ & $2.267 \pm 1.064$ & $-6.120 \pm 0.133$ \\
\hline \multirow[t]{2}{*}{100} & $-4.817 \pm 0.635$ & $-2.614 \pm 0.184$ & $5.595 \pm 0.979$ & $3.415 \pm 0.513$ & $-0.103 \pm 0.437$ & $-1.679 \pm 0.288$ & $0.611 \pm 0.222$ & $2.797 \pm 0.176$ & $-7.930 \pm 0.370$ \\
\hline & \multicolumn{9}{|c|}{ Plantarflexion } \\
\hline 0 & $-0.525 \pm 0.277$ & $2.120 \pm 0.526$ & $-1.712 \pm 0.962$ & $-0.306 \pm 0.207$ & $0.235 \pm 0.062$ & $-4.023 \pm 0.733$ & $-0.743 \pm 0.158$ & $-1.697 \pm 0.091$ & $4.859 \pm 0.845$ \\
\hline 25 & $1.851 \pm 4.896$ & $-0.108 \pm 0.379$ & $-0.254 \pm 0.591$ & $-2.322 \pm 0.730$ & $-1.446 \pm 0.930$ & $-4.925 \pm 3.071$ & $1.642 \pm 1.110$ & $-2.906 \pm 1.982$ & $7.425 \pm 4.524$ \\
\hline 50 & $2.953 \pm 0.146$ & $1.258 \pm 0.894$ & $-2.614 \pm 0.987$ & $-4.897 \pm 0.149$ & $-0.105 \pm 0.303$ & $-3.486 \pm 1.036$ & $0.049 \pm 0.230$ & $-2.518 \pm 0.096$ & $6.146 \pm 0.324$ \\
\hline 75 & $3.984 \pm 0.276$ & $2.648 \pm 0.993$ & $-4.517 \pm 1.154$ & $-0.199 \pm 0.122$ & $1.014 \pm 0.291$ & $0.071 \pm 0.666$ & $2.667 \pm 0.389$ & $-0.658 \pm 0.408$ & $4.642 \pm 0.561$ \\
\hline 100 & $2.681 \pm 0.227$ & $1.234 \pm 0.243$ & $3.266 \pm 0.364$ & $2.473 \pm 0.409$ & $-0.612 \pm 0.909$ & $1.615 \pm 1.353$ & $-0.889 \pm 1.608$ & $-1.015 \pm 0.607$ & $2.661 \pm 3.087$ \\
\hline$\%$ & \multicolumn{9}{|c|}{ Rotation (degrees) \pm Standard Deviation } \\
\hline vGRF & \multicolumn{9}{|c|}{ Dorsiflexion } \\
\hline AT & \multicolumn{3}{|c|}{ Calcaneus } & \multicolumn{3}{|c|}{ Talus } & \multicolumn{3}{|c|}{ Navicular } \\
\hline Load & $\mathrm{X}$ & $\mathrm{Y}$ & $\mathrm{Z}$ & $\mathrm{X}$ & $\mathrm{Y}$ & $\mathrm{Z}$ & $\mathrm{X}$ & $\mathrm{Y}$ & $\mathrm{Z}$ \\
\hline 0 & $-0.449 \pm 0.282$ & $1.413 \pm 2.658$ & $-0.514 \pm 0.353$ & $-0.021 \pm 0.650$ & $1.802 \pm 1.127$ & $-0.289 \pm 0.165$ & $0.061 \pm 0.233$ & $-0.288 \pm 0.112$ & $0.053 \pm 0.029$ \\
\hline 25 & $-4.574 \pm 1.347$ & $3.827 \pm 0.702$ & $0.324 \pm 0.132$ & $1.481 \pm 0.490$ & $4.823 \pm 0.670$ & $0.569 \pm 0.217$ & $1.058 \pm 0.131$ & $0.584 \pm 0.288$ & $0.432 \pm 0.291$ \\
\hline 50 & $1.833 \pm 0.623$ & $-2.418 \pm 1.948$ & $0.053 \pm 0.103$ & $3.353 \pm 2.045$ & $5.289 \pm 1.872$ & $1.571 \pm 0.725$ & $2.873 \pm 0.118$ & $0.202 \pm 0.404$ & $0.375 \pm 0.095$ \\
\hline 75 & $-0.209 \pm 0.644$ & $2.916 \pm 1.371$ & $-0.490 \pm 0.447$ & $-1.916 \pm 0.914$ & $1.982 \pm 0.971$ & $0.264 \pm 0.144$ & $1.595 \pm 0.159$ & $0.923 \pm 0.568$ & $0.395 \pm 0.464$ \\
\hline \multirow[t]{2}{*}{100} & $-2.106 \pm 0.309$ & $0.267 \pm 1.154$ & $0.292 \pm 0.134$ & $1.386 \pm 0.870$ & $0.876 \pm 0.275$ & $0.049 \pm 0.311$ & $1.958 \pm 0.144$ & $0.518 \pm 0.191$ & $-0.126 \pm 0.121$ \\
\hline & \multicolumn{9}{|c|}{ Plantarflexion } \\
\hline 0 & $1.092 \pm 0.501$ & $-1.963 \pm 0.361$ & $0.497 \pm 0.061$ & $-0.734 \pm 0.503$ & $-4.675 \pm 0.070$ & $-0.769 \pm 0.059$ & $-1.102 \pm 0.509$ & $0.211 \pm 0.107$ & $0.160 \pm 0.031$ \\
\hline 25 & $0.110 \pm 1.360$ & $-1.058 \pm 3.364$ & $-0.316 \pm 0.431$ & $3.583 \pm 2.685$ & $-1.248 \pm 0.791$ & $-0.343 \pm 0.173$ & $-1.768 \pm 1.026$ & $0.228 \pm 1.515$ & $0.148 \pm 0.077$ \\
\hline 50 & $1.530 \pm 0.867$ & $-2.196 \pm 0.699$ & $-0.432 \pm 0.096$ & $0.760 \pm 0.660$ & $-2.858 \pm 0.309$ & $-0.782 \pm 0.294$ & $-1.794 \pm 0.150$ & $-0.832 \pm 0.111$ & $-0.017 \pm 0.059$ \\
\hline 75 & $1.701 \pm 0.703$ & $2.206 \pm 0.336$ & $0.419 \pm 0.138$ & $-2.635 \pm 0.797$ & $-2.247 \pm 0.249$ & $-0.866 \pm 0.159$ & $-0.419 \pm 0.176$ & $1.105 \pm 0.350$ & $-0.145 \pm 0.053$ \\
\hline 100 & $1.551 \pm 0.154$ & $1.700 \pm 0.628$ & $0.717 \pm 0.098$ & $0.396 \pm 1.534$ & $0.731 \pm 0.491$ & $0.104 \pm 0.097$ & $-0.799 \pm 0.724$ & $-1.392 \pm 0.858$ & $0.163 \pm 0.229$ \\
\hline
\end{tabular}

Positive rotations in the $\mathrm{X}, \mathrm{Y}$, and $\mathrm{Z}$ axes corresponded to inversion, abduction, and plantarflexion. Positive translations in the $\mathrm{X}$, $\mathrm{Y}$, and $Z$ axes corresponded to anterior, superior, and medial translations. 
Table 4-8. $\quad$ Specimen 4 Translations and Rotations.

\begin{tabular}{|c|c|c|c|c|c|c|c|c|c|}
\hline \multirow{4}{*}{$\begin{array}{c}\% \\
\text { vGRF } \\
\text { AT } \\
\text { Load }\end{array}$} & \multicolumn{9}{|c|}{ Translation $(\mathrm{mm}) \pm$ Standard Deviation } \\
\hline & \multicolumn{9}{|c|}{ Dorsiflexion } \\
\hline & \multicolumn{3}{|c|}{ Calcaneus } & \multicolumn{3}{|c|}{ Talus } & \multicolumn{3}{|c|}{ Navicular } \\
\hline & $\mathrm{X}$ & $\mathrm{Y}$ & $\mathrm{Z}$ & $\mathrm{X}$ & $\mathrm{Y}$ & Z & $\mathrm{X}$ & $\mathrm{Y}$ & Z \\
\hline 0 & $0.867 \pm 0.235$ & $1.624 \pm 0.141$ & $-3.850 \pm 0.502$ & $0.726 \pm 0.122$ & $-0.539 \pm 0.058$ & $-2.290 \pm 0.730$ & $-1.125 \pm 0.110$ & $-2.222 \pm 0.063$ & $-7.212 \pm 0.182$ \\
\hline 25 & $-2.647 \pm 1.404$ & $0.399 \pm 0.274$ & $0.983 \pm 0.830$ & $-3.114 \pm 0.162$ & $0.035 \pm 0.204$ & $2.042 \pm 0.659$ & $5.193 \pm 0.455$ & $-2.443 \pm 0.491$ & $-2.228 \pm 0.713$ \\
\hline 50 & $-3.037 \pm 0.141$ & $0.669 \pm 0.017$ & $-2.085 \pm 0.289$ & $-3.847 \pm 0.506$ & $-0.283 \pm 0.258$ & $3.469 \pm 0.710$ & $5.557 \pm 0.419$ & $-2.412 \pm 0.096$ & $-4.053 \pm 0.554$ \\
\hline 75 & $-2.982 \pm 0.397$ & $0.255 \pm 0.162$ & $-1.256 \pm 0.160$ & $-2.493 \pm 0.058$ & $0.717 \pm 0.127$ & $5.061 \pm 0.431$ & $4.549 \pm 0.784$ & $-0.570 \pm 0.142$ & $1.276 \pm 1.870$ \\
\hline \multirow[t]{2}{*}{100} & $-3.783 \pm 0.677$ & $0.226 \pm 0.113$ & $-2.124 \pm 0.451$ & $-2.772 \pm 0.434$ & $0.659 \pm 0.230$ & $0.406 \pm 2.968$ & $3.529 \pm 0.405$ & $-1.287 \pm 0.711$ & $-3.480 \pm 2.266$ \\
\hline & \multicolumn{9}{|c|}{ Plantarflexion } \\
\hline 0 & $-0.180 \pm 0.129$ & $-0.955 \pm 0.030$ & $2.676 \pm 0.173$ & $-0.131 \pm 0.042$ & $0.424 \pm 0.031$ & $-0.124 \pm 0.141$ & $-7.386 \pm 0.123$ & $-0.373 \pm 0.176$ & $-4.162 \pm 0.453$ \\
\hline 25 & $1.487 \pm 0.403$ & $0.613 \pm 0.045$ & $-2.418 \pm 0.606$ & $2.640 \pm 0.495$ & $-0.363 \pm 0.102$ & $-8.262 \pm 0.850$ & $-1.104 \pm 0.111$ & $2.115 \pm 0.286$ & $4.142 \pm 1.983$ \\
\hline 50 & $2.633 \pm 0.592$ & $0.099 \pm 0.183$ & $-2.125 \pm 0.592$ & $2.504 \pm 0.436$ & $0.498 \pm 0.512$ & $-0.541 \pm 0.592$ & $-1.469 \pm 0.432$ & $0.155 \pm 0.595$ & $0.078 \pm 2.360$ \\
\hline 75 & $0.042 \pm 0.649$ & $0.314 \pm 0.076$ & $-2.277 \pm 1.109$ & $2.739 \pm 0.041$ & $-0.128 \pm 0.172$ & $-3.296 \pm 3.751$ & $-1.102 \pm 0.847$ & $0.426 \pm 0.197$ & $0.421 \pm 0.720$ \\
\hline 100 & $-0.762 \pm 0.262$ & $-0.385 \pm 0.216$ & $-0.434 \pm 2.773$ & $1.531 \pm 0.650$ & $-1.489 \pm 0.581$ & $-0.105 \pm 6.115$ & $-0.242 \pm 1.231$ & $-0.003 \pm 0.744$ & $-1.177 \pm 1.904$ \\
\hline$\%$ & \multicolumn{9}{|c|}{ Rotation (degrees) \pm Standard Deviation } \\
\hline vGRF & \multicolumn{9}{|c|}{ Dorsiflexion } \\
\hline $\mathrm{AT}$ & \multicolumn{3}{|c|}{ Calcaneus } & \multicolumn{3}{|c|}{ Talus } & \multicolumn{3}{|c|}{ Navicular } \\
\hline Load & $\mathrm{X}$ & $\mathrm{Y}$ & $\mathrm{Z}$ & $\mathrm{X}$ & $\mathrm{Y}$ & $\mathrm{Z}$ & $\mathrm{X}$ & $\mathrm{Y}$ & $\mathrm{Z}$ \\
\hline 0 & $1.557 \pm 0.238$ & $0.229 \pm 0.110$ & $-0.649 \pm 0.136$ & $1.722 \pm 0.456$ & $0.421 \pm 0.229$ & $-0.336 \pm 0.044$ & $0.266 \pm 0.024$ & $-4.278 \pm 0.070$ & $0.582 \pm 0.032$ \\
\hline 25 & $-1.249 \pm 0.171$ & $0.232 \pm 1.062$ & $-0.165 \pm 0.332$ & $-0.713 \pm 0.121$ & $0.195 \pm 0.626$ & $0.184 \pm 0.032$ & $-0.139 \pm 0.644$ & $-1.150 \pm 0.464$ & $1.436 \pm 0.144$ \\
\hline 50 & $0.921 \pm 0.119$ & $-0.259 \pm 0.193$ & $-0.334 \pm 0.039$ & $-0.553 \pm 0.427$ & $0.464 \pm 0.977$ & $0.740 \pm 0.045$ & $0.977 \pm 0.707$ & $-0.149 \pm 0.325$ & $0.752 \pm 0.729$ \\
\hline 75 & $0.307 \pm 0.147$ & $0.431 \pm 0.221$ & $0.456 \pm 0.138$ & $-0.823 \pm 0.363$ & $2.171 \pm 0.377$ & $0.290 \pm 0.031$ & $-1.406 \pm 0.607$ & $-0.662 \pm 0.410$ & $1.058 \pm 0.214$ \\
\hline \multirow[t]{2}{*}{100} & $0.923 \pm 0.222$ & $-0.389 \pm 0.414$ & $0.374 \pm 0.224$ & $1.178 \pm 2.274$ & $0.539 \pm 0.334$ & $0.123 \pm 0.060$ & $-0.454 \pm 1.019$ & $-2.337 \pm 0.229$ & $1.023 \pm 0.031$ \\
\hline & \multicolumn{9}{|c|}{ Plantarflexion } \\
\hline 0 & $-1.057 \pm 0.052$ & $-0.300 \pm 0.036$ & $0.271 \pm 0.070$ & $0.262 \pm 0.095$ & $0.804 \pm 0.086$ & $0.190 \pm 0.038$ & $1.915 \pm 0.213$ & $-0.103 \pm 0.015$ & $-0.883 \pm 0.056$ \\
\hline 25 & $1.101 \pm 0.213$ & $-0.115 \pm 0.211$ & $0.514 \pm 0.118$ & $4.260 \pm 0.333$ & $0.171 \pm 1.303$ & $0.125 \pm 0.170$ & $-0.259 \pm 0.720$ & $2.101 \pm 0.304$ & $-1.550 \pm 0.070$ \\
\hline 50 & $0.735 \pm 0.357$ & $1.388 \pm 0.299$ & $0.220 \pm 0.165$ & $0.212 \pm 0.815$ & $1.708 \pm 1.097$ & $-0.575 \pm 0.211$ & $0.709 \pm 0.796$ & $0.835 \pm 0.584$ & $-0.621 \pm 0.109$ \\
\hline 75 & $1.094 \pm 0.566$ & $-0.518 \pm 0.277$ & $0.709 \pm 0.073$ & $1.458 \pm 1.853$ & $0.102 \pm 0.621$ & $0.029 \pm 0.099$ & $1.322 \pm 0.403$ & $1.867 \pm 0.038$ & $-0.940 \pm 0.247$ \\
\hline 100 & $-0.284 \pm 0.378$ & $-1.371 \pm 0.166$ & $0.729 \pm 0.262$ & $-3.405 \pm 3.089$ & $-1.837 \pm 1.076$ & $0.198 \pm 0.200$ & $1.802 \pm 1.078$ & $1.298 \pm 0.123$ & $-0.851 \pm 0.209$ \\
\hline
\end{tabular}

Positive rotations in the $\mathrm{X}, \mathrm{Y}$, and $\mathrm{Z}$ axes corresponded to eversion, adduction, and dorsiflexion. Positive translations in the $\mathrm{X}$, $\mathrm{Y}$, and

$\mathrm{Z}$ axes corresponded to anterior, superior, and lateral translations. 
translation. In general, translational and rotational measurements through dorsiflexion and plantarflexion were minimal (within a few degrees and millimeters) and can be deemed surgically insignificant. Most motion occurred in the navicular in the coronal plane: the navicular translated laterally in dorsiflexion and medially in plantarflexion. Virtually no relative motion occurred between the calcaneus and talus in the sagittal or coronal planes (MMS X and Z axes); both translated posteriorly and medially during dorsiflexion and anteriorly and laterally during plantarflexion. The navicular appeared to be at a maximum height in the neutral vertical orientation and translated inferiorly through degrees of motion. This demonstrated the arches of the foot are activated, such that the navicular reached its maximum height and could not translate any direction except inferiorly.

\section{Discussion}

The goal of this work was to define relative kinematics of the talus, calcaneus, navicular, and articular joint surfaces to better define motion at the ankle during stance phase gait. A novel dynamic robotic testing platform and protocol were used to simulate loading conditions representative of in vivo conditions of early stance phase gait. The simulation accounted for 10 degrees of plantarflexion and dorsiflexion, whereas the total functional range of walking motion of the ankle during stance phase is approximately 15 degrees $[2,4,37,39]$. Force errors were controlled within prescribed $\pm 3 \mathrm{~N}$ tolerances throughout testing. Application of the AT force was within four degrees of the long axis of the tibia introduced a minimal force error of $0.25 \%$. Axial tibia forces used to simulate physiologic vGRF through gait were reduced to 534N due to the load capacity of the upper load cell. However, these approximated BW forces were within $18 \mathrm{~N}$ of the physiologic weight of specimens 1 and 2 , and within $71 \mathrm{~N}$ for specimens 3 and 4 . This accounts for at most $10.6 \%$ error in force application $(25 \%$ AT vGRF loading condition for specimens 3 and 4).

Minimal AT loads (25\% vGRF) had a significant effect on the axis of rotation, causing a superior shift in the IAR of more than $10 \mathrm{~mm}$ for all specimens. Once the AT was loaded, no definitive effect of increasing load was demonstrated except for in dorsiflexion where the increasing load shifted the XIAR paths posteriorly. This suggests that the foot ankle complex may have intraspecimen variability between cycles of motion even when forces and kinematic patterns are tightly controlled. Additionally, net joint loading once the arch was formed by applying an AT force had no effect on the IAR of the ankle. Dorsiflexion paths, in general, showed little movement in the talus. In plantarflexion, the first degrees of motion started up within the articular surface of the talus. As rotation continued, the IAR path moved downward towards the middle of the talus, transitioning from a fixed point rotation at the joint articular surfaces to a sliding motion between them, moving closer to the rotational center as defined by the arc of the tibiotalar articulating surfaces. Specimen 1 and 2 showed less movement within the talus, whereas specimen 3 and 4 showed a much higher range of movement. This confirms interspecimen variability. Stability of the ankle joint is determined by three 
main factors: articular congruity, ligamentous structures, and ankle position [11,36]. All have been shown to have high variability between specimens [51].

Bone motion analyses demonstrated little movement throughout dorsiflexion and plantarflexion. Most rotations and translations were less than a few degrees or millimeters and were deemed surgically insignificant. However, relative translation between the calcaneus and talus was minimal in the coronal and sagittal planes. In vitro and in vivo studies have reported relative motion between these bones predominantly during heel strike and heel rise [20,25,44,46,58]. This protocol does not account for those portions of stance phase gait and thus is in agreement with current literature. More motion in the three bones occurred in the coronal plane (Z-axis). The navicular demonstrated a lateral translation throughout dorsiflexion and a medial translation in plantarflexion. The navicular also demonstrated a slight inferior translation throughout dorsiflexion and plantarflexion. This can be interpreted as a confirmation of arch formation due to an AT load.

Some limitations were present in this study. This current model does not incorporate a dynamic Achilles tendon force profile representative of in vivo loading conditions, nor does it account for any of the other major plantarflexors, extrinsic dorsiflexors, or intrinsic muscles of the foot. However, these muscles are predominantly active during heel strike, and heel rise to toe off gait $[4,11,36,37,39]$. The force applied via the AT has been shown to have the greatest role in the biomechanical behavior of the ankle joint during stance phase when the foot is flat and therefore, it is the only tendon force accounted for in this study. During normal gait, the tibia is driven from maximum plantarflexion to dorsiflexion with a continuous load on the joint. This protocol is limited in that the dorsiflexion and plantarflexion paths are generated independently.

Furthermore, plantarflexion is driven in a direction uncharacteristic of the target in vivo stance phase motion. However, the Achilles tendon load is applied to the specimen in such a manner to simulate the in vivo loading scenario where the joint is plantarflexed. The independent movement from the neutral orientation between dorsi- and plantarflexion may explain the disconnect between the initial point of IAR paths in dorsiflexion and plantarflexion, as well as the high starting point for the plantarflexion IAR path forcing the ligaments and tissue to stabilize in the neutral orientation. Another limitation is that our study was two-dimensional whereas the ankle joint can move in three dimensions $[1,11,50]$. Finally, the MMS data was analyzed in the anatomical cardinal planes and do not necessarily reflect the axes of rotation of joints. However, because motion was primarily in the sagittal plane, this limitation is minor. Additionally, because rotations were minimal, transforming the translational data to a joint center was unnecessary.

In conclusion, this work has provided a two-dimensional analysis of the IAR of the ankle joint with a corresponding three-dimensional bone motion analysis using a novel robotic testing platform and protocol. The results of the study are one of a handful to address the IAR of the ankle joint. This yielded great insight into the biomechanical properties of the foot and ankle, including the tibiotalar relationship on the axis of rotation and the effect of load on foot-ankle kinematics. Current work includes 
incorporating a dynamic Achilles tendon load and demonstrating the effects of shoe support or tendon pathologies on ankle biomechanics. 


\section{CHAPTER 5. DISCUSSION}

A novel dynamic robotic testing platform and protocol were developed and used to investigate the biomechanical behavior of the foot and ankle under simulated loading conditions representative of in vivo conditions of early stance phase gait. The objectives of the current work were to: (1) Design a RTP and loading protocol that supported in vitro cadaveric testing of the foot and ankle complex during the early stance phase of gait, (2) Assess the repeatability and accuracy of the loading protocol using force measurements and IAR data from two cadaver specimens, and (3) Study the effects of a passive (unloaded) and active Achilles load and axial tibia load on ankle mechanics using IAR data and translational and rotational data of the calcaneus, talus, and navicular from four cadaver specimens during stance phase gait.

The first objective of this study was to validate the accuracy and repeatability of a loading protocol. The model simulated a static Achilles load during stance phase of walking with decreased in vivo loading conditions. The role of soft tissue in this model was accounted for by preconditioning of the cadaver feet by subjecting them to several load and movement cycles before relevant data were captured. The simulation accounted for 20 degrees of plantarflexion and 10 degrees dorsiflexion, whereas the total functional range of walking motion of the ankle during stance phase is approximately 15 degrees $[3,27,37,39]$. While force errors were typically controlled within prescribed $\pm 2 \mathrm{~N}$ tolerances throughout testing, they increased towards the end of motion possibly as a result of rotating the ankle beyond its functional limit. The ATLs were limited by the upper loading capacity of the gimbal load cell, such that loading conditions were set at half body weight (356N) and the Achilles tendon force was set to half the vGRF (178N). However, the ground reaction forces produced during the simulation were in agreement with a percentage of recorded in vivo forces [1-4,11,39]. Additionally, application of the AT force to within four degrees of the long axis of the tibia introduced a minimal force error of $0.25 \%$.

The repeatability of the loading protocol was addressed by assessing the intraspecimen variability between cycles of motion. Stability of the ankle joint is determined by three main factors: articular congruity, ligamentous structures, and ankle position $[11,36]$. All have been shown to have high variability between specimens [51]. Baxter showed a difference in biomechanical properties of sprinters versus non sprinters, suggesting physical health plays a major role in mechanics of the ankle [35]. Therefore, the goal was to demonstrate a repeatable measurement of the IAR in the sagittal plane. This loading protocol showed variability in the mean IAR measurements between cycles of motion (as measured by the standard deviation) of less than $1 \mathrm{~mm}$. The talus dimension for the two specimens were approximately $60 \mathrm{~mm}$ in width and $38 \mathrm{~mm}$ in height, demonstrating small relative variability on a clinical scale. Current gait simulators and BTP protocols are either unable to measure the IAR [14-22,24] or are limited to high errors in the calculation of the IAR due to methodology $[23,35]$. While dorsiflexion paths remained within a $4.5 \mathrm{~mm}$ range, plantarflexion paths showed more movement. However, plantarflexion slopes were not significantly different between 
cycles of motion in specimen 1 or 2 , demonstrating a repeatable measurement of the IAR path.

The second objective of this work was to define relative kinematics of the talus, calcaneus, navicular, and articular joint surfaces to better define motion at the ankle during stance phase gait. The novel RTP and protocol were used to simulate loading conditions representative of in vivo conditions of early stance phase gait. The simulation accounted for 10 degrees of plantarflexion and dorsiflexion, whereas the total functional range of walking motion of the ankle during stance phase is approximately 15 degrees $[2,4,37,39]$. Force errors were controlled within prescribed $\pm 2 \mathrm{~N}$ tolerances throughout testing. Application of the AT force was within four degrees of the long axis of the tibia introduced a minimal force error of $0.25 \%$. Axial tibia forces used to simulate physiologic vGRF through gait were reduced to 534N due to the load capacity of the upper load cell. However, these approximated BW forces were within $18 \mathrm{~N}$ of the physiologic weight of specimens 1 and 2, and within $71 \mathrm{~N}$ for specimens 3 and 4 . This accounts for at most 11.7 percent error in force application (25\% AT vGRF loading condition for specimens 3 and 4).

Minimal AT loads (25\% vGRF) had a significant effect on the axis of rotation, causing a superior shift in the IAR of more than $10 \mathrm{~mm}$ for all specimens. Once the AT was loaded, no definitive effect of increasing load was demonstrated except for in dorsiflexion where the increasing load shifted the XIAR paths posteriorly. This suggests that the foot ankle complex may have intraspecimen variability between cycles of motion even when forces and kinematic patterns are tightly controlled. Additionally, net joint loading, once the arch was formed by applying an AT force, had no effect on the IAR of the ankle. Dorsiflexion paths, in general, showed little movement in the talus. In plantarflexion, the first degrees of motion started up within the articular surface of the talus. As rotation continued, the IAR path moved downward towards the middle of the talus, transitioning from a fixed point rotation at the joint articular surfaces to a sliding motion between them, moving closer to the rotational center as defined by the arc of the tibiotalar articulating surfaces. Specimen 1 and 2 showed less movement within the talus, whereas specimen 3 and 4 showed a much higher range of movement. This confirms interspecimen variability. Stability of the ankle joint is determined by three main factors: articular congruity, ligamentous structures, and ankle position [11,36]. All have been shown to have high variability between specimens [51].

Bone motion analyses demonstrated little movement throughout dorsiflexion and plantarflexion. Most rotations and translations were less than a few degrees or millimeters and were deemed surgically insignificant. However, relative translation between the calcaneus and talus was minimal in the coronal and sagittal planes. In vitro and in vivo studies have reported relative motion between these bones predominantly during heel strike and heel rise [20,25,44,46,58]. This protocol does not account for those portions of stance phase gait and thus is in agreement with current literature. More motion in the three bones occurred in the coronal plane (Z-axis). The navicular demonstrated a lateral translation throughout dorsiflexion and a medial translation in plantarflexion. The navicular also demonstrated a slight inferior translation throughout 
dorsiflexion and plantarflexion. This can be interpreted as a confirmation of arch formation due to an AT load.

Some limitations are present in this study. The proximal fusion of the tibia and fibula removed the physiologic joint function where the bones may undergo translations and rotations relative to each other. This has been observed primarily when the knee experiences external and internal rotation [28]. Our model accounts for tibial sagittal plane motion only. During force application an out of plane load was produced as a mode of lateral stabilization of the joint. However, a negligible moment build up was observed throughout motion $(0.5 \mathrm{Nm})$ and did not restrict flexion. Additionally, the current model does not incorporate a dynamic Achilles tendon force profile representative of in vivo loading conditions, nor does it account for any of the other major plantarflexors, extrinsic dorsiflexors, or intrinsic muscles of the foot. However, these muscles are predominantly active during heel strike, and heel rise to toe off gait [36,39]. The force applied via the Achilles tendon has been shown to have the greatest role in the biomechanical behavior of the ankle joint during stance phase when the foot is flat. Therefore, it is the only tendon force accounted for in this study. During normal gait, the tibia is driven from maximum plantarflexion to dorsiflexion with a continuous load on the joint. This protocol is limited in that the dorsiflexion and plantarflexion paths are generated independently. Furthermore, plantarflexion is driven in a direction uncharacteristic of the target in vivo stance phase motion. However, the Achilles tendon load is applied to the specimen in such a manner to simulate the in vivo loading scenario where the joint is plantarflexed. The independent movement from the neutral orientation between dorsi- and plantar-flexion may explain the disconnect between the initial point of IAR paths in dorsiflexion and plantarflexion, as well as the high starting point for the plantarflexion IAR path forcing the ligaments and tissue to stabilize in the neutral orientation. Another limitation is that our study was two-dimensional whereas the ankle joint can move in three dimensions $[1,11,50]$. Finally, the MMS data were analyzed in the anatomical cardinal planes and do not necessarily reflect the axes of rotation of joints. However, because motion was primarily in the sagittal plane, this limitation is minor. Additionally, because rotations were minimal, transforming the translational data to a joint center was unnecessary. 


\section{CHAPTER 6. CONCLUSION}

In conclusion, this work has provided description and validation of a novel loading protocol developed to support in vitro cadaveric testing of the foot and ankle complex. This protocol provided a two-dimensional analysis of the IAR of the ankle joint using tightly controlled loads in conjunction with a passive AT force. The low force error tolerance and consistent axial force values demonstrated the RTP's ability to accurately simulate forces. The results of the study are one of a handful to address the instantaneous axis of rotation of the ankle joint and provide the most accurate measurement to date. The IAR measurement was repeatable within one millimeter, smaller than what is feasibly measurable in a clinical setting. This yielded great insight into the biomechanical properties of the foot and ankle, including the tibiotalar relationship on the axis of rotation and the effect of load on foot-ankle kinematics. Because the IAR measurement was a direct representation of soft tissue structures and articular geometry, future in vitro studies may yield great insight into the biomechanical properties of the foot and ankle within the sagittal plane, including arch formation and effects of orthotics and footwear on ankle kinematics. As new information describing the dynamic loading characteristics of the foot and ankle is obtained from in vivo gait studies (stair climbing, orthotics, footwear), this novel RTP and test protocol can readily simulate these conditions. Current development includes incorporating a dynamic Achilles tendon load, and demonstrating the effects of shoe support or tendon pathologies on ankle biomechanics. 


\section{LIST OF REFERENCES}

1. Bishop, C., Paul, G., Thewlis, D. (2011). Defining standards for modelling the biomechanics of the foot and ankle: a systematic review. J Foot Ankle Res. 4(Suppl 1), O9.

2. Hamill, J., Knutzen, K.M. (2006). Biomechanical basis of human movement, second ed. New York: Lippincott Williams \& Wilkins.

3. Kirtley, C. (2006). Clinical gait analysis: Theory and practice. New York: Elsevier Churchill Livingstone.

4. Nordin, M., Frankel, V.H. (2001). Basic biomechanics of the musculoskeletal system, third edition. Philadelphia: Lippincott Williams \& Wilkins.

5. DiDomenico, L.A., Camasta, C.A. (2010). Is total ankle replacement more effective than ankle arthrodesis? Podiatry Today $23,50-8$.

6. Saltzman, C.L., Mann, R.A., Ahrens, J.E., Amendola, A., Anderson, R.B, Berlet, G.C., et al. (2009). Prospective controlled trial of STAR total ankle replacement versus ankle fusion: initial results. Foot Ankle Intl. 30(7), 579-596.

7. Piriou, P., Culpan, P., Mullins, M., Cardon, J.N., Pozzi, D., Judet, T. (2008). Ankle replacement versus arthrodesis: a comparative gait analysis study. Foot Ankle Intl. 29(1), 3-9.

8. Gougoulias, N.E., Khanna, A., Maffulli, N. (2009). History and evolution in total ankle arthroplasty. Br. Med. Bull. 89(1), 111-151.

9. Pedowitz, D.I., Wapner, K.L. (2010). Total ankle replacement: Where do we stand? UPOJ. 20, 97-99.

10. Blitz, N. (2012). Contemporary controversies in foot and ankle surgery, an issue of clinics in podiatric medicine and surgery, Vol. 29. No. 3. Elsevier.

11. Castro, M.D. (2002). Ankle biomechanics. Foot Ankle Clin. N. AM. 7, 679-693.

12. Lieberman, D. E., Venkadesan, M., Werbel, W. A., Daoud, A. I., D’Andrea, S., Davis, I. S., ... \& Pitsiladis, Y. (2010). Foot strike patterns and collision forces in habitually barefoot versus shod runners. Nature, 463(7280), 531-535.

13. Aquino, A., Payne, C. (1999). Function of the plantar fascia. The Foot 9(2), 7378. 
14. Nester, C.J., Liu, A.M., Ward, E., Howard, D., Cocheba, J., Derrick, T., Patterson, P. (2007). In vitro study of foot kinematics using a dynamic walking cadaver model. J. Biomech. 40(2), 1927-1937.

15. Suckel, A., Muller, O., Langenstein, P., Herberts, T., Reize, P., Wulker, N. (2008). Chopart's joint load during gait: In vitro study of 10 cadaver specimen in a dynamic model. Gait and Posture 27, 216-222.

16. Noble Jr., L.D., Colbrunn, R.W., Lee, D.G., van den Bogert, A.J., Davis, B.L. (2010). Design and validation of a general purpose robotic testing system for musculoskeletal applications. J Biomech Eng. 132(2), 025001:1-12.

17. Burg, J., Peeters, K., Natsakis, T., Deretmaeker, G., Sloten, J.V. (2013). In vitro analysis of muscle activity illustrates mediolateral decoupling of hind and mid foot bone motion. Gait \& Posture 38(1), 56-61.

18. Kim, K., Kitaoka, H.B., Luo, Z.P., Ozeki, S., Berglund, L.J., Kaufman, K.R., An, K.N. (2001). In Vitro Simulation of the Stance Phase in Human Gait. Journal of Musculoskeletal Research 5(6), 113-122.

19. Watanabe, K., Kitaoka, H.B., Fujii, T., Crevoisier, X., Beglund, L.J., Zhao, K.D., Kaufman, K.R., An, K.N. (2013). Posterior tibial tendon dysfunction and flatfoot: Analysis with simulated walking. Gait \& Posture 37(2), 264-268.

20. Whittaker, E.C., Aubin, P.M., Ledoux, W.R. (2011). Foot bone kinematics as measured in a cadaver robotic gait simulator. Gait \& Posture 33, 645-650.

21. Sharkey, N.A., Hamel, A.J. (1998). A dynamic cadaver model of the stance phase of gait: performance characteristics and kinetic validation. Clin. Biomech. $13,420-433$.

22. Hurschler, C., Emmerich, J., Wulker, N. (2003). In vitro simulation of stance phase gait - Part I: Model verification. Foot \& Ankle Intl. 24(8), 614-622.

23. Sumiya T, Suzuki Y, Kasagara, T., Ogata, H. (1997). Instantaneous centers of rotation in dorsi/plantar flexion movements of posterior-type plastic ankle-foot orthosis. J. of Rehab. Research \& Dev. 34(3), 279-285.

24. Bahr, R., Pena, F., Shine, J., Lew, W.D., Engebretsen, L. (1998). Ligament force and joint motion in the intact ankle: a cadaveric study.Knee Surg Sports Traumatol Arthrosc. 6(2), 115-21.

25. Hansen, M.L., Otis, J.C., Kenneally, S.M., Deland, J.T. (2001). A closed loop cadaveric foot and ankle loading model. J. of Biomech. 34, 551-555. 
26. Thordarson, D.B., Schmotzer, H., Chon, J., Peters, J. (1995). Dynamic support of the human longitudinal arch: A biomechanical evaluation. Clin. Orthopaedics and Related Research 316, 165-172.

27. Jarrell, S.E., Owen, J.R., Wayne, J.S., Adelaar, R.S., 2009. Biomechanical comparison of screw versus plate/screw construct for talonavicular fusion. Foot \& Ankle Intl. 30(2), 150-156.

28. Scott, J., Lee, H., Barsoum, W., Van Den Bogert, A.J. (2007). The effect of tibiofemoral loading on proximal tibiafibular joint motion. J of Anatomy 211(5), 647-653.

29. Okita, N., Meyers, S. A., Challis, J. H., Sharkey, N. A. (2009). An objective evaluation of a segmented foot model. Gait \& Posture 30(1), 27-34.

30. Sammarco, G.J., Burstein, A.H., Frankel, V.H. (1973). Biomechanics of the ankle: a kinematic study. Orthop. Clin. North Am. 4(1), 75-96.

31. Leardini, A., O'Connor, J.J., Catani, F., Giannini, S. (1999). A geometric model of the human ankle joint. J Biomech. 32(6), 585-591.

32. Leardini, A. (2001). Geometry and mechanics of the human ankle complex and ankle prosthesis design. Clin Biomech. 16(8), 706-709.

33. Leardini, A., O'Connor, J.J., Catani, F., Giannini, S. (1999). Kinematics of the human ankle complex in passive flexion; a single degree of freedom system. $\mathrm{J}$ Biomech. 32(2), 111-118.

34. Leardini, A., O'Connor, J.J., Catani, F., Giannini, S. (2004). Mobility of the human ankle and the design of total ankle replacement. Clin Orthop Relat Res. 424, 39-46.

35. Baxter, J.R., Novack, T.A., Van Werkhoven, H., Pennell, D.R., Piazza, S.J. (2012). Ankle joint mechanics and foot proportions differ between human sprinters and non-sprinters. Proc Biol Sci. 22(1735), 2018-2024.

36. Kelikian, A.S., Sarrafian, S. (2011). Sarrafian's anatomy of the foot and ankle: Descriptive, topographic, functional, third edition. Lippincott Williams \& Wilkins, New York.

37. Winter, D.A. (2009). Biomechanics and motor control of human movement, fourth edition. Wiley, Hoboken.

38. Maganaris, C.N., Baltzopoulos, V., Sargeant, A.J. (1999). Changes in Achilles tendon moment arm from rest to maximum isometric plantarflexion: in vivo observations in man. J. Physiol. 510, 977-985. 
39. Rogers, M.M. (1988). Dynamic biomechanics of the normal foot and ankle during walking and running. Physical Therapy. 68 (12), 1822-1830.

40. Goto, A., Moritoma, H. Itohara, T., Watanabe, T., Sugamoto, K., (2009). Three dimensional in vivo kinematics of the subtalar joint during dorsi-plantarflexion and inversion-eversion. Foot Ankle Int. 30: 432-438.

41. Arndt, A., Westblad, P., Winson, I., Hashimoto, T., Lundberg, A. (2004). Ankle and subtalar kinematics measured with intracortical pins during the stance phase of walking. Foot \& Ankle Intl. 25(5), 357-364.

42. Arndt, A., Wolf, P., Liu, A., Nester, C., Stacoff, A., Jones, R., Lundberg, A. (2007). Intrinsic foot kinematics measured in vivo during the stance phase of slow running. J of Biomech. 40(12), 2672-2678.

43. Lundberg, A. (1989). Kinematics of the ankle and foot: in vivo roentgen stereophotogrammetry. Acta Orthopaedica 60(S233), 1-26.

44. Lundgren, P., Nester, C., Liu, A., Arndt, A., Jones, R., Stacoff, A., Lundberg, A. (2008). Invasive in vivo measurement of rear-, mid-and forefoot motion during walking. Gait \& Posture 28(1), 93-100.

45. Mattingly, B., Talwalkar, V., Tylkowski, C., Stevens, D. B., Hardy, P. A., Pienkowski, D. (2006). Three-dimensional in vivo motion of adult hind foot bones. J of Biomech. 39(4), 726-733.

46. Nester, C., Jones, R. K., Liu, A., Howard, D., Lundberg, A., Arndt, A., Wolf, P. (2007). Foot kinematics during walking measured using bone and surface mounted markers. J of Biomech. 40(15), 3412-3423.

47. Roche, C., Mattingly, B., Talwalkar, V., Tylkowski, C., Stevens, D. B., Hardy, P. A., Pienkowski, D. (2005). Three-dimensional hindfoot motion in adolescents with surgically treated unilateral clubfoot. J of Pediatric Orthop. 25(5), 630-634.

48. Siegler, S., Udupa, J. K., Ringleb, S. I., Imhauser, C. W., Hirsch, B. E., Odhner, D., Roach, N. (2005). Mechanics of the ankle and subtalar joints revealed through a 3D quasi-static stress MRI technique. J of Biomech. 38(3), 567-578.

49. Wearing, S.C., Smeathers, J.E., Yates, B., Sullivan, P.M., Urry, S.R., Dubois, P. (2005). Errors in measuring sagittal arch kinematics of the human foot with digital fluoroscopy. Gait \& Posture 21(3), 326-332.

50. Nester, C.J. (2009). Lessons from dynamic cadaver and invasive bone pin studies: do we know how the foot really moves? J of Foot and Ankle Res. 2, 18. 
51. Kleipool, R.P., Blankevoort, L. (2010). The relation between geometry and function of the ankle joint complex: a biomechanical review. Knee Surg. Sports Traumatol. Arthrosc. 18: 618-627.

52. Gray, H. and Lewis, W.H. (1918). Anatomy of the human body. (pp.268). Philadelphia: Lea \& Febiger.

53. Crisco III, J.J., Chen, X., Panjabi, M.M., Wolfe, S.W., (1994). Optimal marker placement for calculating instantaneous center of rotation. J Biomech. 27(9), 1183-1187.

54. Stewart, T.E. (2012). An innovative testing protocol to study foot and ankle kinetics during early stance phase of gait. Master's Thesis, UTHSC.

55. Semple, R., Murley, G.S., Woodburn, J., Turner, D.E. (2009). Tibialis posterior in health and disease: a review of structure and function with specific reference to electromyographic studies. J of Foot and Ankle Res. 2, 24.

56. Kelly, B.P., DiAngelo, D.J. (2013). A multi-axis programmable robot for the study of multi -body spine biomechanics using a real-time trajectory path modification force and displacement control strategy. Accepted for publication in the ASME Journal of Medical Devices, February 2013.

57. Leardini, A., Benedetti, M. G., Berti, L., Bettinelli, D., Nativo, R., \& Giannini, S. (2007). Rear-foot, mid-foot and fore-foot motion during the stance phase of gait.Gait \& posture, 25(3), 453-462.

58. Hamel, A. J., Sharkey, N. A., Buczek, F. L., \& Michelson, J. (2004). Relative motions of the tibia, talus, and calcaneus during the stance phase of gait: a cadaver study. Gait \& posture, 20(2), 147-153.

59. Ghotge, R.S. (2010). Effect of fixation using locked compression plate versus lag screws on biomechanics of talonavicular joint: a human cadaveric model. Master's Thesis, UTHSC. 


\section{APPENDIX A. $\quad$ RADIOGRAPHS AND TRANSFORMATIONS}

Specimen radiographs are shown in Figure A-1. Specimen 1 and 2 were the left and right lower legs of a male age 37, and specimen 3 and 4 were of a female age 44 . Body weight was $552 \mathrm{~N}$ (124 lbs) for the male subject and $605 \mathrm{~N}$ (136 lbs) for the female. The extended tool tip and generic axes transformations have been previously discussed (Chapter 3). Figure A-2 shows the possible offset of the ETT from the generic axes. Table A-1 shows the ETT and generic axis transformations for all specimens with respect to the bisector at the top of the potting material. The $\mathrm{z}$ offset from the NTT to the top of the potting material is $-187.2 \mathrm{~mm}$ for the $445 \mathrm{~N}$ (1001b) load cell and $-172.2 \mathrm{~mm}$ for the $578.5 \mathrm{~N}(130 \mathrm{lb})$ load cell. This is added to the generic axis offset and together they are subtracted from the IAR data to obtain the IAR values on the generic axis. Figure A-3 shows radiographic measurements of the specimen for comparison. Table A-2 shows the measurements of the talus, including lengths, widths, and radii of the talar dome with respective ratios to a generic model talus. 


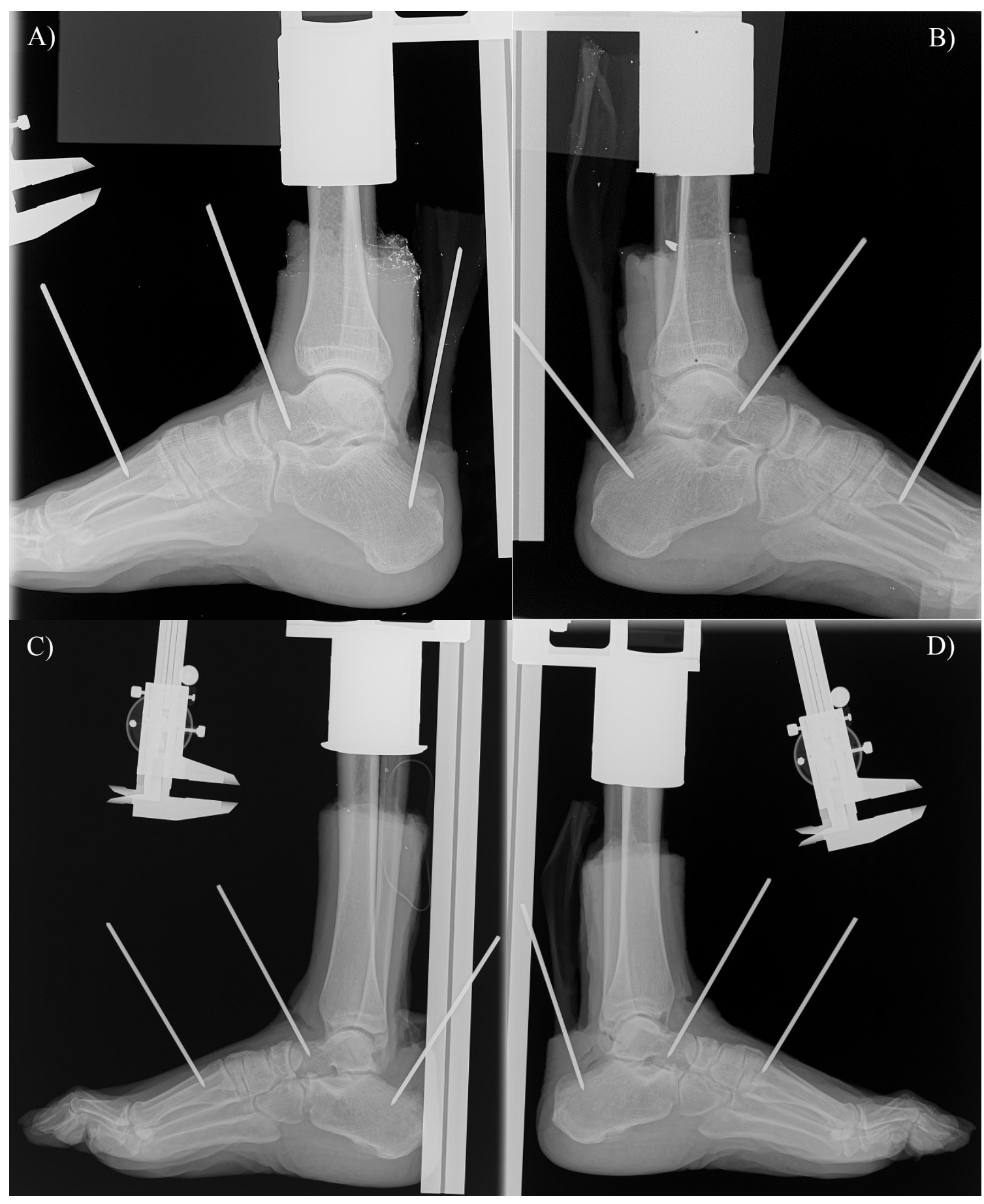

Figure A-1. Specimen Radiographs.

Radiographs of A) specimen 1, B) specimen 2, C) specimen 3, and D) specimen 4. Not to scale. However, calipers were set to $10 \mathrm{~mm}$ and placed in field of view for scaling. All measurements were done in ImageJ $(\mathrm{NIH})$. Additional radiographs were taken after navicular k-wires were inserted. 


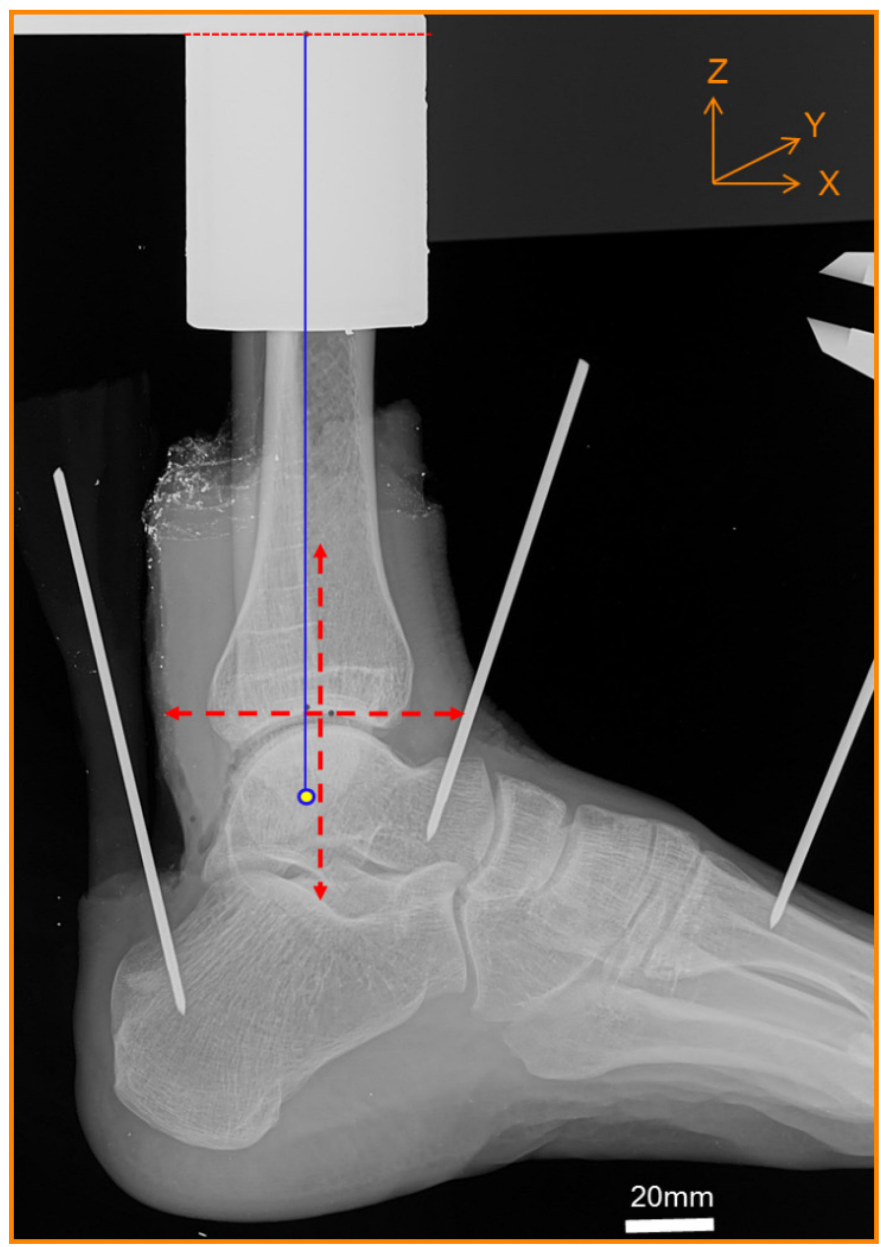

Figure A-2. Example of Extended Tool Tip and Generic Axis Transformations. The ETT transform is designated as the vertical offset from the potting material bisector located in the center of the talus (blue solid line, yellow dot). The generic axes are located at the meeting point of the bisector of the tibia and highest point on the tibial plafond (red dashed axes).

Table A-1. Transformation Values for Program Input and Generic Axis Offsets

\begin{tabular}{cccccc}
\hline \multirow{2}{*}{ Specimen } & \multicolumn{2}{c}{ ETT Transforms } & & \multicolumn{2}{c}{ Generic Axis Transforms } \\
\cline { 2 - 3 } \cline { 5 - 6 } & $\begin{array}{c}\text { X Offset } \\
(\mathrm{mm})\end{array}$ & $\begin{array}{c}\text { Z Offset } \\
(\mathrm{mm})\end{array}$ & & $\begin{array}{c}\text { X Offset } \\
(\mathrm{mm})\end{array}$ & $\begin{array}{c}\text { Z Offset } \\
(\mathrm{mm})\end{array}$ \\
\hline 1 & 0 & -171 & & 2.47 & -148.02 \\
2 & 0 & -177 & & 3.16 & -155.23 \\
3 & 0 & -235 & & 6.93 & -219.9 \\
4 & 0 & -209 & & 6.38 & -190.43 \\
\hline
\end{tabular}

Note: Transformations are with respect to the top of the bisector of the potting material. 


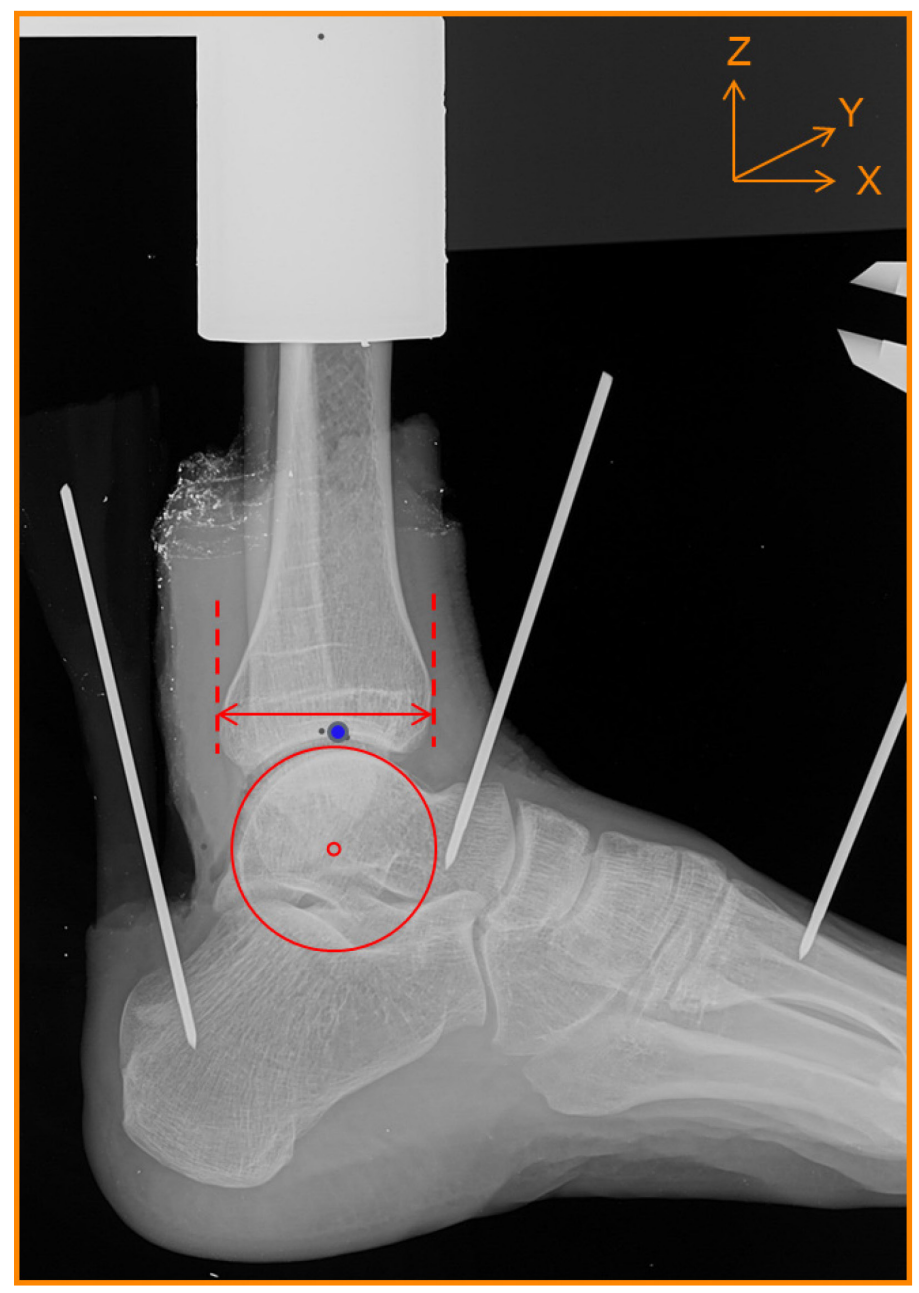

Figure A-3. Talus Measurements.

Generic axis point is shown. Maximum tibia with is shown with the red solid arrow spanning between the dotted red lines. Diameter was measured by placing a circle that matched the arc of the talar dome. Lengths and widths of the talus are direct measurements of maximum measurements.

Table A-2. Talus Measurements and Ratio Comparison to Generic Model

\begin{tabular}{ccccccc}
\hline Specimen & $\begin{array}{c}\text { Width } \\
(\mathrm{mm})\end{array}$ & $\begin{array}{c}\text { Height } \\
(\mathrm{mm})\end{array}$ & $\begin{array}{c}\text { Circular } \\
\text { Diameter } \\
(\mathrm{mm})\end{array}$ & $\begin{array}{c}\text { Maximum } \\
\text { Tibia Width } \\
(\mathrm{mm})\end{array}$ & $\begin{array}{c}\text { Circular } \\
\text { Ratio }\end{array}$ & $\begin{array}{c}\text { Tibia } \\
\text { Width } \\
\text { Ratio }\end{array}$ \\
\hline Generic & 60.21 & 26.97 & 46.45 & 42.96 & 1.00 & 1.00 \\
1 & 60.14 & 25.10 & 44.35 & 45.35 & 0.96 & 1.06 \\
2 & 61.57 & 24.91 & 45.25 & 47.18 & 0.97 & 1.10 \\
3 & 47.60 & 20.91 & 30.28 & 39.69 & 0.65 & 0.92 \\
4 & 47.37 & 20.75 & 29.54 & 39.54 & 0.64 & 0.92 \\
\hline
\end{tabular}




\section{APPENDIX B. MOTION MEASURING SYSTEM TRANSFORMATIONS}

Camera/target reference frames followed a left handed system where the X-axis aligned with the world coordinate system (WCS), the Y-axis aligned with the Z-axis of the WCS, and the Z-axis aligns with the negative Y-axis of the WCS. This discussion will follow the convention of the camera system. Camera 1 and 2 were placed 180 degrees apart on either side of the RTP with the X-axis aligned in the sagittal plane. Data were transformed to have coincident reference frames where the positive and negative values match the WCS convention. X rotations follow a clockwise convention, whereas the $\mathrm{Y}$ and $\mathrm{Z}$ axes follow a counterclockwise convention. Targets were initially mounted on both sides of the mounting block in the sagittal plane 180 degrees apart for zeroing. Targets were placed with LED 1 aligned with the $\mathrm{Y}$ axis of the camera system (upright). Targets locations were recorded to obtain exact reference locations of the cameras to each other and also to zero the camera coordinate system about the sagittal plane. Once this data were acquired, targets were mounted on the K-wires in the aforementioned bones (Chapter 4).

$\mathrm{K}$-wire transforms were from the center of the target to the tip of the K-wire. This method has been previously described in detail [59]. Briefly, LED 1 of the target was aligned with the K-wire orientation. "a," was the X offset of the target center from the Kwire in the target reference frame, "l" was the length of the K-wire from the target center to the tip of the K-wire implanted in the bony segment, and "p" was the z offset of the target center from the k-wire in the target reference frame. The length, "l," of the Kwires was determined post-experiment by removing and measuring the marked location of target. Rotational offsets were with respect to the camera reference frame aligned in the sagittal plane. 


\section{APPENDIX C. BONE TRANSLATIONS AND ROTATIONS}

Individual specimen bone kinematic data were presented previously to demonstrate inter-specimen variability and show patterns in bony motion. Table C-1 and Figures $\mathbf{C}-\mathbf{1}$ and $\mathbf{C}-\mathbf{2}$ show the mean combined translational and rotational data for all four specimens. Motion data axes were flipped for left feet to align with a right foot specimen such that positive values reflected the same type of motion for all specimens. Positive rotations in the $\mathrm{X}, \mathrm{Y}$, and $\mathrm{Z}$ axes corresponded to eversion, adduction, and dorsiflexion. Positive translations in the $\mathrm{X}, \mathrm{Y}$, and $\mathrm{Z}$ axes corresponded to anterior, superior, and lateral translations.

Translations were minimal. Maximum values reached $4.5 \mathrm{~mm}$, but remained predominantly under $2 \mathrm{~mm}$. No patterns were exhibited except slight motion of the calcaneus in the $\mathrm{X}$ axis and slight motion of the navicular in the Z-axis. The calcaneus translated posteriorly in dorsiflexion, and anteriorly in plantarflexion. In the mean values, the calcaneus and talus exhibited minor relative motion of a few millimeters. Because of the variability between specimens, the minimal relative motion between the calcaneus and talus was lost in the mean values. The navicular translated laterally in dorsiflexion and medially in plantarflexion. Rotational values had a maximum of 2.3 degrees. In most cases the rotations remained within one degree. 
Table C-1. Mean Translations for All Specimens.

\begin{tabular}{|c|c|c|c|c|c|c|c|c|c|}
\hline \multirow{4}{*}{$\begin{array}{c}\% \\
\text { vGRF } \\
\text { AT } \\
\text { Load }\end{array}$} & \multicolumn{9}{|c|}{ Translation $(\mathrm{mm}) \pm$ Standard Deviation } \\
\hline & \multicolumn{9}{|c|}{ Dorsiflexion } \\
\hline & \multicolumn{3}{|c|}{ Calcaneus } & \multicolumn{3}{|c|}{ Talus } & \multicolumn{3}{|c|}{ Navicular } \\
\hline & $\mathrm{X}$ & $\mathrm{Y}$ & $\mathrm{Z}$ & $\mathrm{X}$ & $\mathrm{Y}$ & $\mathrm{Z}$ & $\mathrm{X}$ & $\mathrm{Y}$ & $\mathrm{Z}$ \\
\hline 0 & $0.946 \pm 1.794$ & $0.425 \pm 0.835$ & $-1.519 \pm 1.759$ & $-0.222 \pm 0.754$ & $0.137 \pm 0.453$ & $-1.834 \pm 1.747$ & $-0.346 \pm 0.990$ & $-0.578 \pm 1.269$ & $-1.108 \pm 4.842$ \\
\hline 25 & $-3.323 \pm 0.773$ & $-0.616 \pm 2.756$ & $-2.317 \pm 3.737$ & $-0.784 \pm 3.993$ & $0.534 \pm 0.542$ & $0.081 \pm 2.275$ & $1.417 \pm 3.215$ & $-0.881 \pm 1.614$ & $1.733 \pm 2.960$ \\
\hline 50 & $-4.426 \pm 1.007$ & $0.815 \pm 0.502$ & $-1.136 \pm 1.271$ & $-0.748 \pm 4.633$ & $-0.686 \pm 0.797$ & $-0.487 \pm 2.682$ & $1.179 \pm 4.445$ & $-1.037 \pm 2.827$ & $4.124 \pm 6.269$ \\
\hline 75 & $-3.132 \pm 1.110$ & $0.090 \pm 0.650$ & $-0.519 \pm 0.695$ & $-1.640 \pm 3.771$ & $0.441 \pm 0.614$ & $0.093 \pm 4.294$ & $0.669 \pm 2.607$ & $-0.684 \pm 2.267$ & $2.791 \pm 2.298$ \\
\hline \multirow[t]{2}{*}{100} & $-4.162 \pm 0.789$ & $-1.392 \pm 1.447$ & $-1.154 \pm 4.761$ & $1.646 \pm 2.975$ & $0.049 \pm 0.414$ & $-0.258 \pm 1.592$ & $0.592 \pm 2.087$ & $-0.322 \pm 2.157$ & $3.697 \pm 4.970$ \\
\hline & \multicolumn{9}{|c|}{ Plantarflexion } \\
\hline 0 & $0.166 \pm 0.652$ & $0.374 \pm 1.279$ & $1.948 \pm 0.487$ & $0.016 \pm 0.799$ & $0.557 \pm 0.453$ & $2.121 \pm 1.753$ & $-1.797 \pm 3.811$ & $-1.224 \pm 0.711$ & $-2.402 \pm 3.230$ \\
\hline 25 & $1.0995 \pm 1.107$ & $0.134 \pm 0.535$ & $0.773 \pm 2.436$ & $1.288 \pm 2.417$ & $-0.202 \pm 1.274$ & $0.918 \pm 6.169$ & $0.198 \pm 1.205$ & $-0.444 \pm 2.052$ & $-2.406 \pm 5.046$ \\
\hline 50 & $2.585 \pm 0.298$ & $0.607 \pm 0.629$ & $1.290 \pm 2.320$ & $1.157 \pm 4.066$ & $-0.203 \pm 0.933$ & $2.536 \pm 2.848$ & $-1.082 \pm 1.102$ & $-0.960 \pm 1.189$ & $-1.607 \pm 3.783$ \\
\hline 75 & $2.838 \pm 1.870$ & $1.333 \pm 0.968$ & $0.560 \pm 4.472$ & $1.302 \pm 1.200$ & $-0.534 \pm 1.221$ & $-0.842 \pm 1.872$ & $1.774 \pm 1.918$ & $-0.484 \pm 0.613$ & $-1.055 \pm 3.445$ \\
\hline 100 & $2.052 \pm 1.986$ & $0.782 \pm 0.880$ & $0.849 \pm 3.353$ & $2.600 \pm 1.437$ & $-0.810 \pm 0.498$ & $0.455 \pm 1.639$ & $0.281 \pm 1.240$ & $-1.097 \pm 0.795$ & $-3.196 \pm 2.635$ \\
\hline$\%$ & \multicolumn{9}{|c|}{ Rotation (degrees) \pm Standard Deviation } \\
\hline vGRF & \multicolumn{9}{|c|}{ Dorsiflexion } \\
\hline $\mathrm{AT}$ & \multicolumn{3}{|c|}{ Calcaneus } & \multicolumn{3}{|c|}{ Talus } & \multicolumn{3}{|c|}{ Navicular } \\
\hline Load & $\mathrm{X}$ & $\mathrm{Y}$ & $\mathrm{Z}$ & $\mathrm{X}$ & $\mathrm{Y}$ & $\mathrm{Z}$ & $\mathrm{X}$ & $\mathrm{Y}$ & $\mathrm{Z}$ \\
\hline 0 & $0.803 \pm 0.635$ & $-0.443 \pm 1.110$ & $0.003 \pm 0.680$ & $0.196 \pm 1.255$ & $-0.501 \pm 1.034$ & $-0.170 \pm 0.811$ & $0.132 \pm 0.435$ & $-0.952 \pm 2.234$ & $0.266 \pm 0.272$ \\
\hline 25 & $2.274 \pm 2.488$ & $-0.642 \pm 2.131$ & $0.288 \pm 0.339$ & $-1.204 \pm 0.452$ & $-1.678 \pm 2.542$ & $0.431 \pm 0.309$ & $-1.120 \pm 0.744$ & $-0.568 \pm 0.424$ & $0.581 \pm 0.584$ \\
\hline 50 & $-0.161 \pm 1.315$ & $0.144 \pm 1.596$ & $-0.091 \pm 0.801$ & $-1.413 \pm 1.881$ & $-0.767 \pm 3.021$ & $0.859 \pm 0.492$ & $-1.236 \pm 2.497$ & $-0.351 \pm 0.441$ & $0.735 \pm 0.656$ \\
\hline 75 & $-0.652 \pm 1.379$ & $-0.538 \pm 1.642$ & $-0.008 \pm 0.667$ & $-0.527 \pm 1.691$ & $0.219 \pm 2.016$ & $-0.020 \pm 0.364$ & $-0.551 \pm 1.786$ & $-0.507 \pm 0.448$ & $0.321 \pm 0.578$ \\
\hline 100 & $1.195 \pm 1.174$ & $-0.101 \pm 0.290$ & $0.359 \pm 0.087$ & $-0.002 \pm 1.487$ & $0.052 \pm 0.801$ & $0.305 \pm 0.464$ & $-1.377 \pm 0.810$ & $-0.450 \pm 1.421$ & $0.312 \pm 0.560$ \\
\hline & \multicolumn{9}{|c|}{ Plantarflexion } \\
\hline 0 & $-0.223 \pm 1.298$ & $0.510 \pm 1.202$ & $0.154 \pm 0.764$ & $0.208 \pm 0.437$ & $1.642 \pm 3.257$ & $-0.065 \pm 1.018$ & $1.094 \pm 0.639$ & $0.244 \pm 0.465$ & $-0.380 \pm 0.651$ \\
\hline 25 & $-0.243 \pm 1.125$ & $0.600 \pm 1.232$ & $0.531 \pm 0.727$ & $1.297 \pm 3.569$ & $0.674 \pm 1.155$ & $-0.313 \pm 0.339$ & $0.778 \pm 0.904$ & $1.725 \pm 1.332$ & $-0.586 \pm 0.727$ \\
\hline 50 & $-1.319 \pm 1.599$ & $0.572 \pm 1.953$ & $0.257 \pm 0.544$ & $-0.199 \pm 0.448$ & $2.291 \pm 0.490$ & $-0.674 \pm 0.086$ & $0.384 \pm 1.873$ & $0.287 \pm 0.636$ & $-0.273 \pm 0.462$ \\
\hline 75 & $-0.482 \pm 1.421$ & $-1.135 \pm 0.903$ & $0.540 \pm 0.124$ & $0.812 \pm 1.873$ & $1.009 \pm 0.902$ & $-0.177 \pm 0.627$ & $0.908 \pm 0.371$ & $0.741 \pm 1.299$ & $-0.583 \pm 0.405$ \\
\hline 100 & $-1.589 \pm 0.936$ & $-0.677 \pm 1.373$ & $0.814 \pm 0.230$ & $-0.883 \pm 1.722$ & $-0.201 \pm 1.330$ & $0.077 \pm 0.286$ & $0.843 \pm 0.810$ & $0.161 \pm 1.519$ & $-0.446 \pm 0.441$ \\
\hline
\end{tabular}



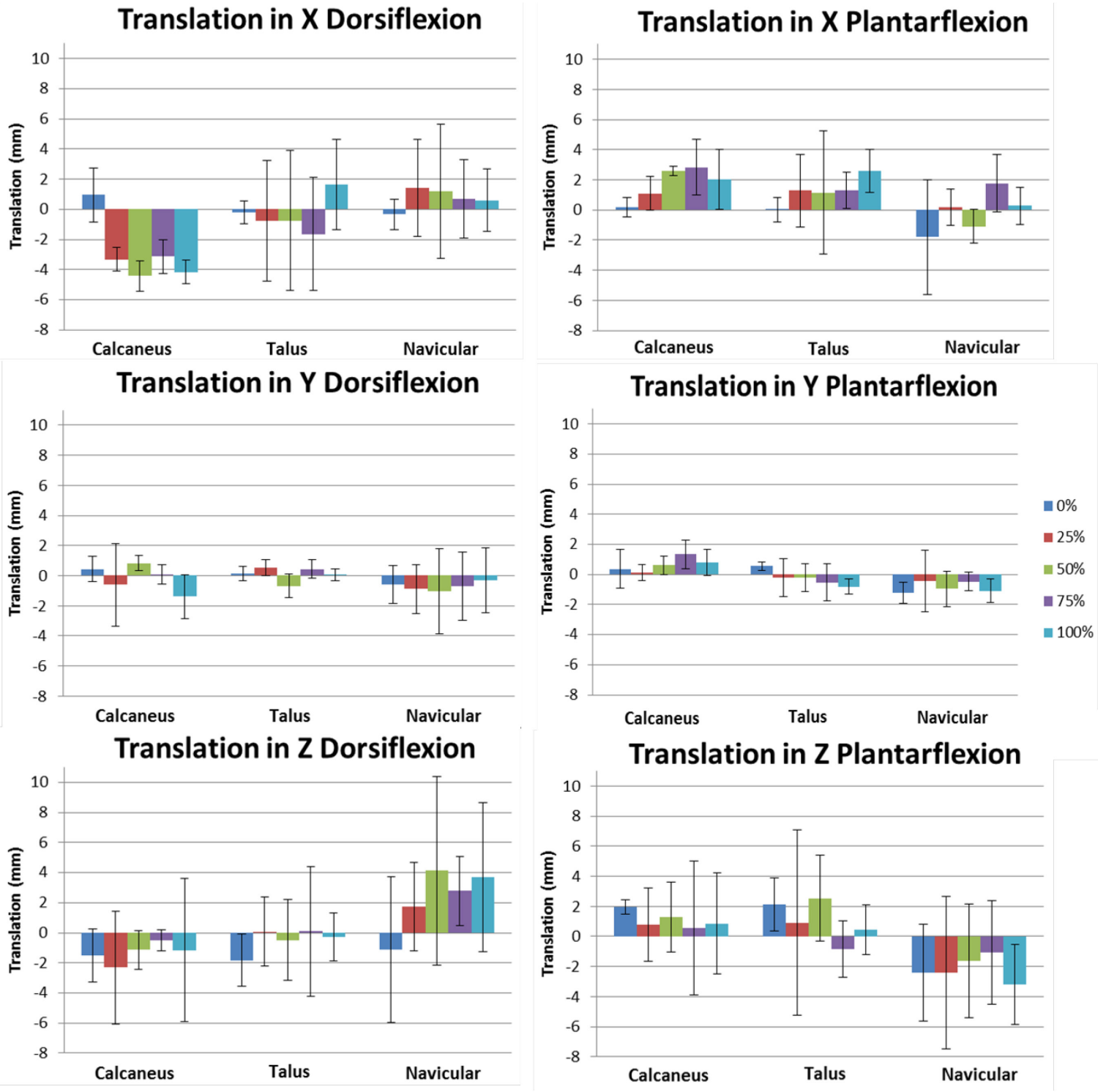

Figure C-1. Bone Translations (Means and Standard Deviations) for All Specimens. 

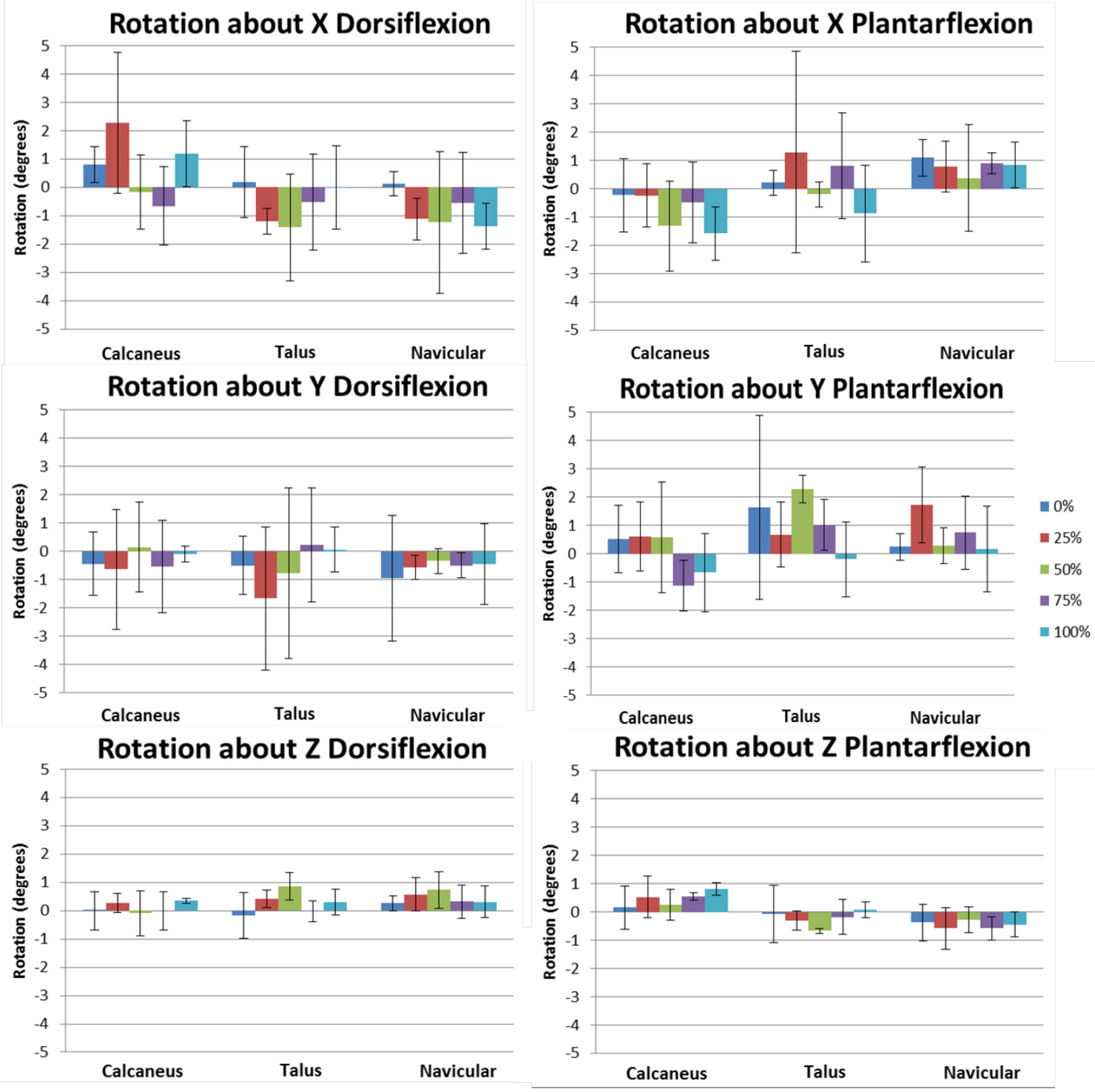

Figure C-2. Bone Rotations (Means and Standard Deviations) for All Specimens. 


\section{APPENDIX D. RAW DATA}

Table D-1. Forces (N) during Motion.

\begin{tabular}{|c|c|c|c|c|c|}
\hline \multicolumn{6}{|c|}{ Dorsiflexion } \\
\hline \multicolumn{3}{|c|}{ Specimen 1} & \multicolumn{3}{|c|}{ Specimen 2} \\
\hline Cycle 1 & Cycle 2 & Cycle 3 & Cycle 1 & Cycle 2 & Cycle 3 \\
\hline-524.536 & -523.836 & -515.265 & -524.361 & -520.775 & -521.956 \\
\hline-526.107 & -526.064 & -513.470 & -520.773 & -515.481 & -525.758 \\
\hline-513.595 & -514.295 & -525.621 & -515.957 & -525.927 & -526.189 \\
\hline-526.968 & -513.15 & -526.137 & -525.656 & -526.356 & -515.249 \\
\hline-513.007 & -514.100 & -514.669 & -521.359 & -515.587 & -515.937 \\
\hline-526.549 & -513.605 & -514.174 & -521.389 & -526.024 & -516.01 \\
\hline-513.501 & -514.463 & -525.045 & -523.515 & -526.051 & -516.562 \\
\hline-511.557 & -513.568 & -514.093 & -517.023 & -51 & 512 \\
\hline-513.108 & -513.939 & -513.283 & -517.394 & -51 & -516.038 \\
\hline-511.640 & -514.657 & -513.651 & -527.338 & 07 & -515.925 \\
\hline-525.736 & & -514.104 & -517.602 & & 897 \\
\hline-520.107 & -514 & -514.379 & $-51 c$ & -51 & 516 \\
\hline-521.866 & -525.364 & -514.039 & -517.187 & -515.963 & -515.788 \\
\hline-511.946 & -513.564 & -514.964 & -524.628 & 532 & -515.445 \\
\hline-513.568 & & & & & -525.681 \\
\hline-513.482 & -514.269 & -524.545 & -51 & 5.144 & -515.581 \\
\hline-512.912 & -514.748 & -514.398 & -517.503 & -526.03 & -516.323 \\
\hline-513.694 & -515.181 & -514.044 & -517.892 & -51 & -515.662 \\
\hline-525.800 & & -514.255 & -51 & 748 & -526.805 \\
\hline & & -514.376 & & & -516.256 \\
\hline & & -517.555 & -518.299 & & -516.987 \\
\hline \multicolumn{6}{|c|}{ Plantarflexion } \\
\hline \multicolumn{3}{|c|}{ Specimen 1} & \multicolumn{3}{|c|}{ Specimen 2} \\
\hline Cycle 1 & & & & & \\
\hline-513.520 & -513.340 & -506.000 & -518.107 & -518.982 & 070 \\
\hline-515.350 & -514.300 & -503.460 & -520.510 & -520.117 & -520.554 \\
\hline-515.170 & -510.450 & -503.230 & -519.717 & -519.543 & -520.811 \\
\hline-504.800 & -504.890 & -515.690 & -520.89 & -520.758 & -520.977 \\
\hline-503.870 & -504.570 & -516.030 & -520.135 & -521.272 & -520.835 \\
\hline-514.700 & -515.490 & -502.240 & -520.777 & -521.258 & -510.807 \\
\hline-515.690 & -515.430 & -517.520 & -520.804 & -511.096 & -510.702 \\
\hline-515.540 & -503.860 & -516.670 & -510.639 & -520.521 & -521.177 \\
\hline-514.510 & -514.460 & -502.960 & -520.63 & -521.198 & -520.673 \\
\hline-504.560 & -504.290 & -515.310 & -510.197 & -520.779 & -510.372 \\
\hline-514.500 & -515.460 & -502.600 & -520.182 & -510.562 & -510.125 \\
\hline
\end{tabular}


Table D-1. (Continued).

\begin{tabular}{cccccccc}
\hline \multicolumn{5}{c}{ Specimen 1 } & & & \multicolumn{3}{c}{ Specimen 2 } \\
\cline { 1 - 3 } \cline { 1 - 1 } Cycle 1 & Cycle 2 & Cycle 3 & & Cycle 1 & Cycle 2 & Cycle 3 \\
\hline-506.900 & -506.110 & -503.580 & & -510.268 & -510.574 & -521.113 \\
-514.650 & -514.960 & -503.590 & & -520.336 & -521.166 & -521.341 \\
-504.120 & -503.860 & -511.510 & & -520.036 & -521.217 & -509.979 \\
-504.120 & -515.230 & -502.240 & & -521.002 & -509.983 & -517.067 \\
-504.390 & -503.600 & -502.460 & & -510.465 & -509.809 & -510.290 \\
-503.600 & -514.490 & -515.710 & & -513.699 & -520.389 & -513.174 \\
-509.060 & -515.620 & -503.330 & & -509.802 & -520.035 & -513.782 \\
-507.700 & -503.800 & -503.060 & & -512.419 & -509.795 & -512.287 \\
-505.940 & -514.950 & -502.090 & & -509.872 & -514.070 & -512.233 \\
-504.040 & -503.300 & -503.170 & & -510.471 & -509.859 & -510.253 \\
-503.680 & -514.440 & -502.190 & & -510.062 & -510.674 & -509.974 \\
-503.400 & -503.050 & -501.250 & & -521.019 & -508.906 & -509.518 \\
-503.900 & -514.220 & -501.670 & & -520.473 & -509.628 & -509.715 \\
-504.140 & -503.350 & -500.860 & & -509.079 & -509.385 & -508.685 \\
-503.500 & -502.620 & -501.790 & & -519.809 & -510.057 & -509.095 \\
-502.860 & -514.180 & -502.420 & & -509.109 & -509.677 & -508.978 \\
-503.440 & -502.910 & -502.950 & & -509.163 & -509.601 & -519.964 \\
-502.880 & -503.790 & -502.880 & & -510.396 & -510.440 & -509.303 \\
-503.060 & -503.270 & -500.430 & & -509.089 & -509.833 & -508.608 \\
-502.840 & -503.540 & -502.840 & & -509.179 & -513.596 & -509.179 \\
-503.670 & -502.580 & -502.620 & & -508.742 & -509.135 & -508.042 \\
-503.270 & -503.620 & -514.900 & & -518.534 & -509.088 & -519.452 \\
-503.090 & -503.570 & -501.430 & & -508.689 & -513.324 & -508.733 \\
-504.350 & -502.560 & -501.860 & & -514.802 & -513.315 & -508.855 \\
-502.590 & -503.730 & -500.930 & & -508.887 & -509.937 & -517.021 \\
-502.270 & -502.970 & -501.830 & & -509.354 & -509.354 & -509.529 \\
-502.340 & -502.300 & -501.290 & & -509.556 & -510.255 & -509.381 \\
-509.580 & -502.450 & -500.920 & & -509.798 & -510.542 & -510.498 \\
-503.170 & -502.950 & -501.730 & & -509.907 & -510.738 & -509.251 \\
-502.540 & -502.230 & -500.920 & & -509.138 & -509.750 & -509.444 \\
\hline
\end{tabular}


Table D-2. Validation Study IAR Values (mm) (Chapter 3).

\begin{tabular}{|c|c|c|c|c|c|c|c|c|c|c|c|}
\hline \multicolumn{12}{|c|}{ Dorsiflexion } \\
\hline \multicolumn{6}{|c|}{ Specimen 1} & \multicolumn{6}{|c|}{ Specimen 2} \\
\hline \multicolumn{2}{|c|}{ Cycle 1} & \multicolumn{2}{|c|}{ Cycle 2} & \multicolumn{2}{|c|}{ Cycle 3} & \multicolumn{2}{|c|}{ Cycle 1} & \multicolumn{2}{|c|}{ Cycle 2} & \multicolumn{2}{|c|}{ Cycle 3} \\
\hline $\mathbf{X}_{\text {IAR }}$ & $\mathbf{Z}_{\text {IAR }}$ & $\mathbf{X}_{\text {IAR }}$ & $\mathbf{Z}_{\mathrm{IAR}}$ & $\mathbf{X}_{\text {IAR }}$ & $\mathbf{Z}_{\text {IAR }}$ & $\mathbf{X}_{\text {IAR }}$ & $\mathbf{Z}_{\text {IAR }}$ & $\mathbf{X}_{\text {IAR }}$ & $\mathbf{Z}_{\text {IAR }}$ & $\mathbf{X}_{\text {IAR }}$ & $\mathbf{Z}_{\text {IAR }}$ \\
\hline-2.82 & -9.20 & -2.72 & -9.78 & -2.80 & -9.35 & -3.32 & -8.07 & -3.63 & -8.95 & -4.00 & -7.99 \\
\hline-2.82 & -9.04 & -2.72 & -9.60 & -2.80 & -9.20 & -3.39 & -7.76 & -3.68 & -8.55 & -4.01 & -7.72 \\
\hline-2.83 & -8.88 & -2.74 & -9.43 & -2.80 & -9.04 & -3.47 & -7.45 & -3.73 & -8.12 & -4.03 & -7.44 \\
\hline-2.83 & -8.75 & -2.75 & -9.28 & -2.80 & -8.92 & -3.56 & -7.16 & -3.78 & -7.73 & -4.05 & -7.19 \\
\hline-2.84 & -8.65 & -2.76 & -9.16 & -2.80 & -8.82 & -3.64 & -6.90 & -3.84 & -7.36 & -4.07 & -6.97 \\
\hline-2.85 & -8.57 & -2.78 & -9.07 & -2.80 & -8.75 & -3.73 & -6.66 & -3.90 & -7.02 & -4.10 & -6.77 \\
\hline-2.87 & -8.52 & -2.80 & -9.00 & -2.81 & -8.70 & -3.82 & -6.46 & -3.97 & -6.71 & -4.12 & -6.60 \\
\hline-2.88 & -8.49 & -2.82 & -8.96 & -2.81 & -8.68 & -3.92 & -6.28 & -4.04 & -6.42 & -4.16 & -6.46 \\
\hline-2.90 & -8.50 & -2.85 & -8.94 & -2.82 & -8.69 & -4.02 & -6.12 & -4.12 & -6.16 & -4.19 & -6.35 \\
\hline-2.91 & -8.52 & -2.87 & -8.95 & -2.83 & -8.72 & -4.12 & -6.00 & -4.20 & -5.93 & -4.23 & -6.26 \\
\hline-2.93 & -8.58 & -2.90 & -8.99 & -2.85 & -8.78 & -4.23 & -5.90 & -4.28 & -5.72 & -4.27 & -6.19 \\
\hline-2.95 & -8.66 & -2.93 & -9.05 & -2.86 & -8.87 & -4.34 & -5.83 & -4.37 & -5.54 & -4.31 & -6.16 \\
\hline-2.98 & -8.76 & -2.96 & -9.14 & -2.87 & -8.98 & -4.45 & -5.79 & -4.46 & -5.39 & -4.36 & -6.15 \\
\hline-3.00 & -8.90 & -2.99 & -9.25 & -2.89 & -9.12 & -4.56 & -5.77 & -4.56 & -5.27 & -4.41 & -6.17 \\
\hline-3.03 & -9.05 & -3.03 & -9.40 & -2.91 & -9.28 & -4.68 & -5.79 & -4.66 & -5.17 & -4.46 & -6.21 \\
\hline-3.06 & -9.24 & -3.06 & -9.56 & -2.93 & -9.47 & -4.80 & -5.83 & -4.76 & -5.10 & -4.51 & -6.28 \\
\hline-3.09 & -9.45 & -3.10 & -9.76 & -2.95 & -9.68 & -4.93 & -5.89 & -4.87 & -5.06 & -4.57 & -6.38 \\
\hline-3.12 & -9.69 & -3.14 & -9.98 & -2.98 & -9.93 & -5.06 & -5.99 & -4.99 & -5.04 & -4.63 & -6.51 \\
\hline-3.15 & -9.95 & -3.18 & -10.23 & -3.00 & -10.20 & -5.19 & -6.11 & -5.10 & -5.06 & -4.70 & -6.66 \\
\hline-3.19 & -10.24 & -3.23 & -10.50 & -3.03 & -10.49 & -5.32 & -6.26 & -5.23 & -5.09 & -4.76 & -6.84 \\
\hline
\end{tabular}


Table D-2. (Continued).

\begin{tabular}{|c|c|c|c|c|c|c|c|c|c|c|c|}
\hline \multicolumn{12}{|c|}{ Plantarflexion } \\
\hline \multicolumn{6}{|c|}{ Specimen 1} & \multicolumn{6}{|c|}{ Specimen 2} \\
\hline \multicolumn{2}{|c|}{ Cycle 1} & \multicolumn{2}{|c|}{ Cycle 2} & \multicolumn{2}{|c|}{ Cycle 3} & \multicolumn{2}{|c|}{ Cycle 1} & \multicolumn{2}{|c|}{ Cycle 2} & \multicolumn{2}{|c|}{ Cycle 3} \\
\hline $\mathbf{X}_{\text {IAR }}$ & $\mathbf{Z}_{\text {IAR }}$ & $\mathbf{X}_{\text {IAR }}$ & $\mathbf{Z}_{\text {IAR }}$ & $\mathbf{X}_{\text {IAR }}$ & $\mathbf{Z}_{\text {IAR }}$ & $\mathbf{X}_{\text {IAR }}$ & $\mathbf{Z}_{\text {IAR }}$ & $\mathbf{X}_{\text {IAR }}$ & $\mathbf{Z}_{\text {IAR }}$ & $\mathbf{X}_{\text {IAR }}$ & $\mathbf{Z}_{\text {IAR }}$ \\
\hline-0.98 & -3.90 & -1.08 & -5.24 & -0.99 & -3.44 & -2.30 & 0.95 & -2.40 & 3.51 & -2.41 & 1.49 \\
\hline-0.90 & -3.83 & -1.02 & -5.12 & -0.91 & -3.38 & -2.22 & 1.04 & -2.29 & 3.51 & -2.31 & 1.56 \\
\hline-0.80 & -3.77 & -0.95 & -5.02 & -0.82 & -3.34 & -2.13 & 1.13 & -2.16 & 3.49 & -2.21 & 1.62 \\
\hline-0.71 & -3.74 & -0.88 & -4.94 & -0.72 & -3.33 & -2.03 & 1.18 & -2.04 & 3.44 & -2.10 & 1.64 \\
\hline-0.62 & -3.73 & -0.81 & -4.89 & -0.63 & -3.34 & -1.94 & 1.21 & -1.91 & 3.36 & -1.99 & 1.64 \\
\hline-0.53 & -3.76 & -0.74 & -4.87 & -0.53 & -3.38 & -1.84 & 1.22 & -1.78 & 3.25 & -1.87 & 1.62 \\
\hline-0.43 & -3.81 & -0.67 & -4.88 & -0.43 & -3.45 & -1.74 & 1.19 & -1.66 & 3.12 & -1.76 & 1.57 \\
\hline-0.34 & -3.88 & -0.60 & -4.91 & -0.33 & -3.54 & -1.65 & 1.14 & -1.53 & 2.96 & -1.65 & 1.49 \\
\hline-0.24 & -3.98 & -0.52 & -4.96 & -0.23 & -3.66 & -1.55 & 1.07 & -1.40 & 2.78 & -1.53 & 1.38 \\
\hline-0.15 & -4.11 & -0.44 & -5.05 & -0.13 & -3.80 & -1.44 & 0.96 & -1.28 & 2.57 & -1.42 & 1.25 \\
\hline-0.05 & -4.27 & -0.36 & -5.15 & -0.03 & -3.98 & -1.34 & 0.83 & -1.15 & 2.33 & -1.30 & 1.09 \\
\hline 0.05 & -4.45 & -0.28 & -5.29 & 0.07 & -4.18 & -1.24 & 0.67 & -1.02 & 2.06 & -1.19 & 0.90 \\
\hline 0.15 & -4.66 & -0.20 & -5.45 & 0.17 & -4.40 & -1.13 & 0.49 & -0.89 & 1.77 & -1.07 & 0.69 \\
\hline 0.25 & -4.89 & -0.12 & -5.64 & 0.28 & -4.66 & -1.03 & 0.28 & -0.77 & 1.45 & -0.95 & 0.45 \\
\hline 0.35 & -5.15 & -0.03 & -5.86 & 0.38 & -4.93 & -0.92 & 0.04 & -0.64 & 1.10 & -0.83 & 0.18 \\
\hline 0.46 & -5.44 & 0.05 & -6.10 & 0.48 & -5.24 & -0.81 & -0.22 & -0.51 & 0.73 & -0.71 & -0.12 \\
\hline 0.56 & -5.76 & 0.14 & -6.37 & 0.59 & -5.57 & -0.70 & -0.52 & -0.38 & 0.33 & -0.59 & -0.44 \\
\hline 0.67 & -6.10 & 0.23 & -6.66 & 0.70 & -5.93 & -0.59 & -0.83 & -0.25 & -0.10 & -0.46 & -0.79 \\
\hline 0.77 & -6.46 & 0.32 & -6.98 & 0.81 & -6.32 & -0.48 & -1.18 & -0.12 & -0.56 & -0.34 & -1.16 \\
\hline 0.88 & -6.86 & 0.41 & -7.33 & 0.91 & -6.73 & -0.37 & -1.55 & 0.01 & -1.04 & -0.21 & -1.56 \\
\hline 0.99 & -7.28 & 0.50 & -7.71 & 1.02 & -7.17 & -0.25 & -1.95 & 0.14 & -1.55 & -0.09 & -1.99 \\
\hline 1.10 & -7.73 & 0.60 & -8.11 & 1.13 & -7.63 & -0.14 & -2.38 & 0.27 & -2.08 & 0.04 & -2.45 \\
\hline
\end{tabular}


Table D-2. (Continued).

\begin{tabular}{|c|c|c|c|c|c|c|c|c|c|c|c|}
\hline \multicolumn{12}{|c|}{ Plantarflexion } \\
\hline \multicolumn{6}{|c|}{ Specimen 1} & \multicolumn{6}{|c|}{ Specimen 2} \\
\hline \multicolumn{2}{|c|}{ Cycle 1} & \multicolumn{2}{|c|}{ Cycle 2} & \multicolumn{2}{|c|}{ Cycle 3} & \multicolumn{2}{|c|}{ Cycle 1} & \multicolumn{2}{|c|}{ Cycle 2} & \multicolumn{2}{|c|}{ Cycle 3} \\
\hline $\mathbf{X}_{\text {IAR }}$ & $\mathbf{Z}_{\text {IAR }}$ & $\mathbf{X}_{\text {IAR }}$ & $\mathbf{Z}_{\mathrm{IAR}}$ & $\mathbf{X}_{\text {IAR }}$ & $\mathbf{Z}_{\text {IAR }}$ & $\mathbf{X}_{\text {IAR }}$ & $\mathbf{Z}_{\mathrm{IAR}}$ & $\mathbf{X}_{\text {IAR }}$ & $\mathbf{Z}_{\text {IAR }}$ & $\mathbf{X}_{\text {IAR }}$ & $\mathbf{Z}_{\text {IAR }}$ \\
\hline 1.21 & -8.20 & 0.69 & -8.54 & 1.25 & -8.13 & -0.02 & -2.83 & 0.40 & -2.64 & 0.17 & -2.93 \\
\hline 1.32 & -8.70 & 0.79 & -8.99 & 1.36 & -8.64 & 0.10 & -3.31 & 0.53 & -3.23 & 0.29 & -3.44 \\
\hline 1.43 & -9.23 & 0.89 & -9.47 & 1.47 & -9.19 & 0.22 & -3.81 & 0.66 & -3.85 & 0.42 & -3.98 \\
\hline 1.54 & -9.78 & 0.99 & -9.98 & 1.58 & -9.76 & 0.34 & -4.35 & 0.79 & -4.49 & 0.55 & -4.54 \\
\hline 1.66 & -10.37 & 1.09 & -10.51 & 1.70 & -10.36 & 0.46 & -4.91 & 0.92 & -5.16 & 0.68 & -5.13 \\
\hline 1.77 & -10.97 & 1.19 & -11.07 & 1.81 & -10.98 & 0.58 & -5.49 & 1.05 & -5.86 & 0.82 & -5.75 \\
\hline 1.89 & -11.61 & 1.29 & -11.66 & 1.93 & -11.64 & 0.70 & -6.10 & 1.18 & -6.58 & 0.95 & -6.39 \\
\hline 2.01 & -12.27 & 1.40 & -12.27 & 2.05 & -12.31 & 0.83 & -6.74 & 1.32 & -7.33 & 1.08 & -7.06 \\
\hline 2.12 & -12.95 & 1.51 & -12.91 & 2.16 & -13.02 & 0.96 & -7.41 & 1.45 & -8.11 & 1.22 & -7.76 \\
\hline 2.24 & -13.67 & 1.61 & -13.58 & 2.28 & -13.75 & 1.08 & -8.11 & 1.58 & -8.91 & 1.36 & -8.49 \\
\hline 2.36 & -14.41 & 1.72 & -14.27 & 2.40 & -14.51 & 1.21 & -8.83 & 1.71 & -9.75 & 1.49 & -9.24 \\
\hline 2.48 & -15.18 & 1.83 & -14.99 & 2.52 & -15.29 & 1.34 & -9.57 & 1.85 & -10.60 & 1.63 & -10.02 \\
\hline 2.61 & -15.97 & 1.95 & -15.73 & 2.64 & -16.10 & 1.47 & -10.35 & 1.98 & -11.49 & 1.77 & -10.82 \\
\hline 2.73 & -16.79 & 2.06 & -16.51 & 2.77 & -16.94 & 1.60 & -11.15 & 2.11 & -12.40 & 1.91 & -11.66 \\
\hline 2.85 & -17.64 & 2.18 & -17.30 & 2.89 & -17.81 & 1.74 & -11.97 & 2.25 & -13.34 & 2.05 & -12.52 \\
\hline 2.98 & -18.51 & 2.29 & -18.13 & 3.01 & -18.70 & 1.87 & -12.83 & 2.38 & -14.30 & 2.19 & -13.40 \\
\hline 3.11 & -19.41 & 2.41 & -18.98 & 3.14 & -19.61 & 2.01 & -13.71 & 2.51 & -15.30 & 2.34 & -14.32 \\
\hline 3.23 & -20.34 & 2.53 & -19.86 & 3.26 & -20.56 & 2.15 & -14.62 & 2.65 & -16.32 & 2.48 & -15.26 \\
\hline
\end{tabular}


Table D-3. Kinematic Study IAR Values ( $\mathrm{mm})$ for Specimen 1.

\begin{tabular}{|c|c|c|c|c|c|c|c|c|c|}
\hline \multicolumn{10}{|c|}{ Dorsiflexion } \\
\hline \multicolumn{2}{|c|}{ Passive (0\%) } & \multicolumn{2}{|c|}{$25 \%$} & \multicolumn{2}{|c|}{$50 \%$} & \multicolumn{2}{|c|}{$75 \%$} & \multicolumn{2}{|c|}{$100 \%$} \\
\hline $\mathbf{X}_{\text {IAR }}$ & $\mathbf{Z}_{\mathrm{IAR}}$ & $\mathbf{X}_{\text {IAR }}$ & $\mathbf{Z}_{\text {IAR }}$ & $\mathbf{X}_{\text {IAR }}$ & $\mathbf{Z}_{\text {IAR }}$ & $\mathbf{X}_{\text {IAR }}$ & $\mathbf{Z}_{\text {IAR }}$ & $\mathbf{X}_{\text {IAR }}$ & $\mathbf{Z}_{\text {IAR }}$ \\
\hline 1.25 & -19.92 & -2.72 & -6.61 & -2.80 & -5.08 & -3.32 & -6.55 & -3.24 & -6.47 \\
\hline 1.33 & -19.70 & -2.72 & -6.43 & -2.80 & -4.93 & -3.41 & -6.27 & -3.33 & -6.29 \\
\hline 1.42 & -19.48 & -2.74 & -6.26 & -2.80 & -4.77 & -3.52 & -5.98 & -3.43 & -6.13 \\
\hline 1.50 & -19.29 & -2.75 & -6.11 & -2.80 & -4.65 & -3.62 & -5.72 & -3.53 & -5.99 \\
\hline 1.59 & -19.12 & -2.76 & -5.99 & -2.80 & -4.55 & -3.73 & -5.48 & -3.63 & -5.87 \\
\hline 1.67 & -18.98 & -2.78 & -5.90 & -2.80 & -4.48 & -3.84 & -5.28 & -3.74 & -5.79 \\
\hline 1.75 & -18.87 & -2.80 & -5.83 & -2.81 & -4.43 & -3.96 & -5.10 & -3.84 & -5.73 \\
\hline 1.82 & -18.78 & -2.82 & -5.79 & -2.81 & -4.41 & -4.08 & -4.95 & -3.95 & -5.70 \\
\hline 1.90 & -18.72 & -2.85 & -5.77 & -2.82 & -4.42 & -4.20 & -4.82 & -4.06 & -5.70 \\
\hline 1.97 & -18.68 & -2.87 & -5.78 & -2.83 & -4.45 & -4.33 & -4.73 & -4.17 & -5.72 \\
\hline 2.04 & -18.67 & -2.90 & -5.82 & -2.85 & -4.51 & -4.45 & -4.66 & -4.29 & -5.77 \\
\hline 2.11 & -18.69 & -2.93 & -5.88 & -2.86 & -4.60 & -4.58 & -4.61 & -4.40 & -5.85 \\
\hline 2.18 & -18.74 & -2.96 & -5.97 & -2.87 & -4.71 & -4.72 & -4.60 & -4.52 & -5.96 \\
\hline 2.24 & -18.81 & -2.99 & -6.08 & -2.89 & -4.85 & -4.85 & -4.61 & -4.64 & -6.09 \\
\hline 2.30 & -18.90 & -3.03 & -6.23 & -2.91 & -5.01 & -4.99 & -4.65 & -4.76 & -6.25 \\
\hline 2.36 & -19.03 & -3.06 & -6.39 & -2.93 & -5.20 & -5.14 & -4.71 & -4.89 & -6.44 \\
\hline 2.42 & -19.18 & -3.10 & -6.59 & -2.95 & -5.41 & -5.28 & -4.81 & -5.01 & -6.66 \\
\hline 2.48 & -19.35 & -3.14 & -6.81 & -2.98 & -5.66 & -5.43 & -4.93 & -5.14 & -6.90 \\
\hline 2.53 & -19.55 & -3.18 & -7.06 & -3.00 & -5.93 & -5.58 & -5.08 & -5.27 & -7.17 \\
\hline 2.58 & -19.78 & -3.23 & -7.33 & -3.03 & -6.22 & -5.74 & -5.25 & -5.40 & -7.47 \\
\hline
\end{tabular}

\section{Plantarflexion}

\begin{tabular}{|c|c|c|c|c|c|c|c|c|c|}
\hline \multicolumn{2}{|c|}{ Passive $(0 \%)$} & \multicolumn{2}{|c|}{$25 \%$} & \multicolumn{2}{|c|}{$50 \%$} & \multicolumn{2}{|c|}{$75 \%$} & \multicolumn{2}{|c|}{$100 \%$} \\
\hline $\mathbf{X}_{\text {IAR }}$ & $\mathbf{Z}_{\text {IAR }}$ & $\mathbf{X}_{\text {IAR }}$ & $\mathbf{Z}_{\text {IAR }}$ & $\mathbf{X}_{\text {IAR }}$ & $\mathbf{Z}_{\text {IAR }}$ & $\mathbf{X}_{\text {IAR }}$ & $\mathbf{Z}_{\text {IAR }}$ & $\mathbf{X}_{\text {IAR }}$ & $\mathbf{Z}_{\text {IAR }}$ \\
\hline 1.19 & -25.64 & -1.08 & -4.07 & 0.11 & -1.17 & -0.99 & -2.27 & 0.36 & -1.32 \\
\hline 1.13 & -25.23 & -1.02 & -3.95 & 0.19 & -1.21 & -0.91 & -2.21 & 0.38 & -1.38 \\
\hline 1.08 & -24.79 & -0.95 & -3.85 & 0.28 & -1.27 & -0.82 & -2.17 & 0.40 & -1.48 \\
\hline 1.02 & -24.39 & -0.88 & -3.77 & 0.38 & -1.36 & -0.72 & -2.16 & 0.42 & -1.60 \\
\hline 0.97 & -24.00 & -0.81 & -3.72 & 0.47 & -1.47 & -0.63 & -2.17 & 0.44 & -1.75 \\
\hline 0.92 & -23.65 & -0.74 & -3.70 & 0.57 & -1.61 & -0.53 & -2.21 & 0.46 & -1.92 \\
\hline 0.88 & -23.32 & -0.67 & -3.71 & 0.67 & -1.78 & -0.43 & -2.28 & 0.48 & -2.13 \\
\hline 0.85 & -23.02 & -0.60 & -3.74 & 0.77 & -1.97 & -0.33 & -2.37 & 0.51 & -2.36 \\
\hline 0.81 & -22.75 & -0.52 & -3.79 & 0.87 & -2.19 & -0.23 & -2.49 & 0.53 & -2.62 \\
\hline 0.79 & -22.50 & -0.44 & -3.88 & 0.97 & -2.43 & -0.13 & -2.63 & 0.55 & -2.90 \\
\hline 0.76 & -22.28 & -0.36 & -3.98 & 1.07 & -2.71 & -0.03 & -2.81 & 0.58 & -3.21 \\
\hline 0.74 & -22.09 & -0.28 & -4.12 & 1.17 & -3.01 & 0.07 & -3.01 & 0.60 & -3.55 \\
\hline 0.73 & -21.92 & -0.20 & -4.28 & 1.27 & -3.33 & 0.17 & -3.23 & 0.63 & -3.92 \\
\hline
\end{tabular}


Table D-3. (Continued).

\begin{tabular}{|c|c|c|c|c|c|c|c|c|c|}
\hline \multicolumn{10}{|c|}{ Plantarflexion } \\
\hline \multicolumn{2}{|c|}{ Passive (0\%) } & \multicolumn{2}{|c|}{$25 \%$} & \multicolumn{2}{|c|}{$50 \%$} & \multicolumn{2}{|c|}{$75 \%$} & \multicolumn{2}{|c|}{$100 \%$} \\
\hline $\mathbf{X}_{\mathrm{IAR}}$ & $\mathbf{Z}_{\mathrm{IAR}}$ & $\mathbf{X}_{\text {IAR }}$ & $Z_{\text {IAR }}$ & $\mathbf{X}_{\mathrm{IAR}}$ & $\mathbf{Z}_{\mathrm{IAR}}$ & $\mathbf{X}_{\text {IAR }}$ & $\mathbf{Z}_{\mathrm{IAR}}$ & $\mathbf{X}_{\text {IAR }}$ & $\mathbf{Z}_{\mathrm{IAR}}$ \\
\hline 1.19 & -25.64 & -1.08 & -4.07 & 0.11 & -1.17 & -0.99 & -2.27 & 0.36 & -1.32 \\
\hline 1.13 & -25.23 & -1.02 & -3.95 & 0.19 & -1.21 & -0.91 & -2.21 & 0.38 & -1.38 \\
\hline 0.72 & -21.78 & -0.12 & -4.47 & 1.38 & -3.68 & 0.28 & -3.48 & 0.65 & -4.31 \\
\hline 0.72 & -21.67 & -0.03 & -4.69 & 1.48 & -4.06 & 0.38 & -3.76 & 0.68 & -4.73 \\
\hline 0.71 & -21.58 & 0.05 & -4.93 & 1.59 & -4.47 & 0.48 & -4.07 & 0.71 & -5.18 \\
\hline 0.72 & -21.53 & 0.14 & -5.20 & 1.69 & -4.90 & 0.59 & -4.40 & 0.74 & -5.66 \\
\hline 0.73 & -21.50 & 0.23 & -5.49 & 1.80 & -5.36 & 0.70 & -4.76 & 0.76 & -6.16 \\
\hline
\end{tabular}


Table D-4. Kinematic Study IAR Values ( $\mathrm{mm})$ for Specimen 2.

\begin{tabular}{|c|c|c|c|c|c|c|c|c|c|}
\hline \multicolumn{10}{|c|}{ Dorsiflexion } \\
\hline \multicolumn{2}{|c|}{ Passive (0\%) } & \multicolumn{2}{|c|}{$25 \%$} & \multicolumn{2}{|c|}{$50 \%$} & \multicolumn{2}{|c|}{$75 \%$} & \multicolumn{2}{|c|}{$100 \%$} \\
\hline $\mathbf{X}_{\text {IAR }}$ & $\mathbf{Z}_{\mathrm{IAR}}$ & $\mathbf{X}_{\text {IAR }}$ & $\mathbf{Z}_{\text {IAR }}$ & $\mathbf{X}_{\text {IAR }}$ & $\mathbf{Z}_{\text {IAR }}$ & $\mathbf{X}_{\text {IAR }}$ & $\mathbf{Z}_{\text {IAR }}$ & $\mathbf{X}_{\text {IAR }}$ & $\mathbf{Z}_{\text {IAR }}$ \\
\hline 1.16 & -18.28 & -2.86 & -6.58 & -3.00 & -5.57 & -3.42 & -5.99 & -4.04 & -5.91 \\
\hline 1.21 & -18.03 & -2.98 & -6.18 & -3.01 & -5.39 & -3.45 & -5.86 & -4.17 & -5.64 \\
\hline 1.26 & -17.76 & -3.12 & -5.75 & -3.03 & -5.22 & -3.47 & -5.75 & -4.30 & -5.37 \\
\hline 1.32 & -17.52 & -3.26 & -5.34 & -3.05 & -5.07 & -3.50 & -5.66 & -4.43 & -5.12 \\
\hline 1.37 & -17.31 & -3.41 & -4.97 & -3.07 & -4.95 & -3.53 & -5.60 & -4.56 & -4.90 \\
\hline 1.42 & -17.12 & -3.56 & -4.63 & -3.10 & -4.86 & -3.56 & -5.57 & -4.70 & -4.71 \\
\hline 1.47 & -16.96 & -3.71 & -4.31 & -3.12 & -4.79 & -3.60 & -5.57 & -4.84 & -4.54 \\
\hline 1.51 & -16.82 & -3.87 & -4.03 & -3.16 & -4.75 & -3.63 & -5.59 & -4.99 & -4.40 \\
\hline 1.55 & -16.71 & -4.04 & -3.77 & -3.19 & -4.73 & -3.67 & -5.63 & -5.14 & -4.28 \\
\hline 1.59 & -16.63 & -4.20 & -3.54 & -3.23 & -4.74 & -3.70 & -5.71 & -5.29 & -4.19 \\
\hline 1.63 & -16.57 & -4.37 & -3.34 & -3.27 & -4.78 & -3.74 & -5.81 & -5.44 & -4.13 \\
\hline 1.67 & -16.54 & -4.55 & -3.17 & -3.31 & -4.84 & -3.78 & -5.94 & -5.60 & -4.09 \\
\hline 1.70 & -16.53 & -4.72 & -3.04 & -3.36 & -4.93 & -3.82 & -6.09 & -5.76 & -4.07 \\
\hline 1.73 & -16.55 & -4.91 & -2.92 & -3.41 & -5.04 & -3.87 & -6.28 & -5.93 & -4.08 \\
\hline 1.76 & -16.59 & -5.09 & -2.84 & -3.46 & -5.19 & -3.91 & -6.48 & -6.09 & -4.11 \\
\hline 1.78 & -16.66 & -5.28 & -2.79 & -3.51 & -5.35 & -3.96 & -6.72 & -6.26 & -4.17 \\
\hline 1.80 & -16.76 & -5.47 & -2.77 & -3.57 & -5.55 & -4.01 & -6.98 & -6.44 & -4.26 \\
\hline 1.82 & -16.88 & -5.67 & -2.78 & -3.63 & -5.77 & -4.06 & -7.27 & -6.62 & -4.37 \\
\hline 1.84 & -17.03 & -5.87 & -2.81 & -3.70 & -6.02 & -4.11 & -7.58 & -6.80 & -4.51 \\
\hline 1.86 & -17.20 & -6.08 & -2.88 & -3.76 & -6.29 & -4.16 & -7.93 & -6.96 & -4.67 \\
\hline
\end{tabular}

Plantarflexion

\begin{tabular}{|c|c|c|c|c|c|c|c|c|c|}
\hline \multicolumn{2}{|c|}{ Passive (0\%) } & \multicolumn{2}{|c|}{$25 \%$} & \multicolumn{2}{|c|}{$50 \%$} & \multicolumn{2}{|c|}{$75 \%$} & \multicolumn{2}{|c|}{$100 \%$} \\
\hline $\mathbf{X}_{\text {IAR }}$ & $\mathbf{Z}_{\text {IAR }}$ & $\mathbf{X}_{\text {IAR }}$ & $\mathbf{Z}_{\text {IAR }}$ & $\mathbf{X}_{\text {IAR }}$ & $\mathbf{Z}_{\text {IAR }}$ & $\mathbf{X}_{\text {IAR }}$ & $\mathbf{Z}_{\text {IAR }}$ & $\mathbf{X}_{\text {IAR }}$ & $\mathbf{Z}_{\text {IAR }}$ \\
\hline 2.55 & -22.83 & 0.62 & -2.91 & -0.77 & -2.51 & 0.34 & -5.23 & -0.83 & -3.82 \\
\hline 2.51 & -22.39 & 0.65 & -2.84 & -0.71 & -2.44 & 0.44 & -5.17 & -0.76 & -3.83 \\
\hline 2.46 & -21.93 & 0.67 & -2.80 & -0.64 & -2.38 & 0.55 & -5.13 & -0.68 & -3.86 \\
\hline 2.43 & -21.48 & 0.70 & -2.78 & -0.57 & -2.36 & 0.67 & -5.12 & -0.60 & -3.92 \\
\hline 2.39 & -21.07 & 0.74 & -2.79 & -0.50 & -2.36 & 0.80 & -5.13 & -0.51 & -4.01 \\
\hline 2.37 & -20.68 & 0.78 & -2.83 & -0.43 & -2.38 & 0.94 & -5.17 & -0.42 & -4.12 \\
\hline 2.35 & -20.32 & 0.82 & -2.89 & -0.36 & -2.43 & 1.09 & -5.24 & -0.33 & -4.26 \\
\hline 2.33 & -19.98 & 0.87 & -2.98 & -0.29 & -2.51 & 1.25 & -5.33 & -0.24 & -4.42 \\
\hline 2.32 & -19.67 & 0.92 & -3.10 & -0.21 & -2.62 & 1.41 & -5.45 & -0.14 & -4.61 \\
\hline 2.31 & -19.39 & 0.98 & -3.24 & -0.13 & -2.75 & 1.59 & -5.59 & -0.04 & -4.83 \\
\hline 2.30 & -19.13 & 1.03 & -3.40 & -0.05 & -2.91 & 1.77 & -5.77 & 0.07 & -5.08 \\
\hline 2.31 & -18.90 & 1.10 & -3.60 & 0.03 & -3.10 & 1.96 & -5.97 & 0.17 & -5.35 \\
\hline 2.31 & -18.70 & 1.16 & -3.82 & 0.11 & -3.31 & 2.16 & -6.19 & 0.28 & -5.64 \\
\hline
\end{tabular}


Table D-4. (Continued).

\begin{tabular}{|c|c|c|c|c|c|c|c|c|c|}
\hline \multicolumn{10}{|c|}{ Plantarflexion } \\
\hline \multicolumn{2}{|c|}{ Passive (0\%) } & \multicolumn{2}{|c|}{$25 \%$} & \multicolumn{2}{|c|}{$50 \%$} & \multicolumn{2}{|c|}{$75 \%$} & \multicolumn{2}{|c|}{$100 \%$} \\
\hline $\mathbf{X}_{\mathrm{IAR}}$ & $\mathrm{Z}_{\mathrm{IAR}}$ & $\mathbf{X}_{\mathrm{IAR}}$ & $Z_{\text {IAR }}$ & $\mathbf{X}_{\mathrm{IAR}}$ & $\mathbf{Z}_{\mathrm{IAR}}$ & $\mathbf{X}_{\mathrm{IAR}}$ & $\mathbf{Z}_{\mathrm{IAR}}$ & $\mathbf{X}_{\text {IAR }}$ & $\mathbf{Z}_{\mathrm{IAR}}$ \\
\hline 2.32 & -18.52 & 1.23 & -4.06 & 0.19 & -3.55 & 2.37 & -6.45 & 0.39 & -5.97 \\
\hline 2.34 & -18.36 & 1.31 & -4.34 & 0.28 & -3.82 & 2.59 & -6.72 & 0.51 & -6.32 \\
\hline 2.36 & -18.24 & 1.38 & -4.63 & 0.36 & -4.12 & 2.82 & -7.03 & 0.63 & -6.69 \\
\hline 2.39 & -18.14 & 1.47 & -4.96 & 0.45 & -4.44 & 3.05 & -7.36 & 0.75 & -7.10 \\
\hline 2.42 & -18.07 & 1.55 & -5.31 & 0.54 & -4.79 & 3.30 & -7.72 & 0.87 & -7.53 \\
\hline 2.46 & -18.02 & 1.64 & -5.69 & 0.63 & -5.16 & 3.55 & -8.11 & 1.00 & -7.98 \\
\hline 2.50 & -18.00 & 1.73 & -6.05 & 0.72 & -5.56 & 3.81 & -8.52 & 1.13 & -8.42 \\
\hline
\end{tabular}


Table D-5. Kinematic Study IAR Values ( $\mathrm{mm})$ for Specimen 3.

\begin{tabular}{|c|c|c|c|c|c|c|c|c|c|}
\hline \multicolumn{10}{|c|}{ Dorsiflexion } \\
\hline \multicolumn{2}{|c|}{ Passive (0\%) } & \multicolumn{2}{|c|}{$25 \%$} & \multicolumn{2}{|c|}{$50 \%$} & \multicolumn{2}{|c|}{$75 \%$} & \multicolumn{2}{|c|}{$100 \%$} \\
\hline $\mathbf{X}_{\text {IAR }}$ & $\mathbf{Z}_{\text {IAR }}$ & $\mathbf{X}_{\text {IAR }}$ & $\mathbf{Z}_{\text {IAR }}$ & $\mathbf{X}_{\text {IAR }}$ & $\mathbf{Z}_{\text {IAR }}$ & $\mathbf{X}_{\mathrm{IAR}}$ & $\mathbf{Z}_{\text {IAR }}$ & $\mathbf{X}_{\text {IAR }}$ & $\mathbf{Z}_{\text {IAR }}$ \\
\hline 1.73 & -13.24 & -2.73 & -9.75 & -4.99 & -2.87 & -6.13 & -4.59 & -6.61 & 0.79 \\
\hline 1.80 & -13.50 & -2.84 & -8.97 & -4.99 & -2.69 & -6.08 & -4.38 & -6.63 & 0.69 \\
\hline 1.89 & -13.81 & -2.97 & -8.11 & -4.99 & -2.51 & -6.04 & -4.19 & -6.66 & 0.55 \\
\hline 1.98 & -14.16 & -3.11 & -7.27 & -4.99 & -2.36 & -5.99 & -4.03 & -6.69 & 0.39 \\
\hline 2.07 & -14.54 & -3.26 & -6.47 & -4.99 & -2.24 & -5.94 & -3.90 & -6.71 & 0.19 \\
\hline 2.16 & -14.95 & -3.41 & -5.69 & -5.00 & -2.15 & -5.90 & -3.80 & -6.74 & -0.04 \\
\hline 2.26 & -15.38 & -3.57 & -4.95 & -5.01 & -2.09 & -5.86 & -3.73 & -6.77 & -0.29 \\
\hline 2.36 & -15.85 & -3.74 & -4.24 & -5.02 & -2.06 & -5.82 & -3.68 & -6.79 & -0.58 \\
\hline 2.46 & -16.35 & -3.92 & -3.57 & -5.03 & -2.06 & -5.79 & -3.67 & -6.82 & -0.90 \\
\hline 2.57 & -16.88 & -4.11 & -2.92 & -5.04 & -2.09 & -5.76 & -3.69 & -6.84 & -1.24 \\
\hline 2.68 & -17.44 & -4.30 & -2.30 & -5.06 & -2.15 & -5.73 & -3.74 & -6.87 & -1.62 \\
\hline 2.79 & -18.03 & -4.51 & -1.72 & -5.08 & -2.24 & -5.70 & -3.81 & -6.89 & -2.03 \\
\hline 2.90 & -18.65 & -4.72 & -1.17 & -5.10 & -2.37 & -5.67 & -3.92 & -6.91 & -2.47 \\
\hline 3.02 & -19.30 & -4.94 & -0.65 & -5.12 & -2.52 & -5.65 & -4.06 & -6.93 & -2.93 \\
\hline 3.14 & -19.97 & -5.16 & -0.16 & -5.15 & -2.70 & -5.63 & -4.22 & -6.96 & -3.43 \\
\hline 3.26 & -20.68 & -5.40 & 0.29 & -5.17 & -2.91 & -5.61 & -4.42 & -6.98 & -3.96 \\
\hline 3.38 & -21.42 & -5.64 & 0.72 & -5.20 & -3.15 & -5.59 & -4.65 & -7.00 & -4.52 \\
\hline 3.51 & -22.19 & -5.89 & 1.11 & -5.23 & -3.42 & -5.57 & -4.90 & -7.02 & -5.10 \\
\hline 3.64 & -22.99 & -6.15 & 1.47 & -5.27 & -3.72 & -5.56 & -5.19 & -7.04 & -5.72 \\
\hline 3.77 & -23.82 & -6.42 & 1.80 & -5.30 & -4.06 & -5.55 & -5.50 & -7.06 & -6.37 \\
\hline
\end{tabular}

\section{Plantarflexion}

\begin{tabular}{|c|c|c|c|c|c|c|c|c|c|}
\hline \multicolumn{2}{|c|}{ Passive $(0 \%)$} & \multicolumn{2}{|c|}{$25 \%$} & \multicolumn{2}{|c|}{$50 \%$} & \multicolumn{2}{|c|}{$75 \%$} & \multicolumn{2}{|c|}{$100 \%$} \\
\hline $\mathbf{X}_{\text {IAR }}$ & $\mathbf{Z}_{\text {IAR }}$ & $\mathbf{X}_{\text {IAR }}$ & $\mathbf{Z}_{\text {IAR }}$ & $\mathbf{X}_{\text {IAR }}$ & $\mathbf{Z}_{\text {IAR }}$ & $\mathbf{X}_{\text {IAR }}$ & $\mathbf{Z}_{\text {IAR }}$ & $\mathbf{X}_{\text {IAR }}$ & $\mathbf{Z}_{\text {IAR }}$ \\
\hline 4.96 & -9.14 & -0.19 & 1.74 & -1.19 & -3.82 & -2.72 & -1.00 & -3.66 & -2.71 \\
\hline 4.87 & -9.80 & -0.08 & 1.54 & -1.15 & -3.64 & -2.65 & -1.09 & -3.64 & -2.71 \\
\hline 4.76 & -10.57 & 0.03 & 1.30 & -1.10 & -3.47 & -2.57 & -1.21 & -3.62 & -2.74 \\
\hline 4.65 & -11.36 & 0.15 & 1.02 & -1.05 & -3.32 & -2.50 & -1.37 & -3.60 & -2.81 \\
\hline 4.53 & -12.19 & 0.26 & 0.71 & -0.99 & -3.21 & -2.42 & -1.56 & -3.58 & -2.90 \\
\hline 4.41 & -13.04 & 0.37 & 0.37 & -0.94 & -3.13 & -2.35 & -1.78 & -3.56 & -3.03 \\
\hline 4.28 & -13.93 & 0.48 & 0.00 & -0.88 & -3.08 & -2.28 & -2.03 & -3.54 & -3.18 \\
\hline 4.15 & -14.84 & 0.59 & -0.40 & -0.82 & -3.06 & -2.20 & -2.31 & -3.51 & -3.37 \\
\hline 4.01 & -15.79 & 0.70 & -0.83 & -0.76 & -3.07 & -2.13 & -2.62 & -3.49 & -3.58 \\
\hline 3.87 & -16.76 & 0.80 & -1.29 & -0.69 & -3.11 & -2.06 & -2.96 & -3.47 & -3.83 \\
\hline 3.72 & -17.76 & 0.91 & -1.78 & -0.63 & -3.18 & -1.99 & -3.33 & -3.45 & -4.10 \\
\hline 3.56 & -18.80 & 1.01 & -2.30 & -0.56 & -3.28 & -1.92 & -3.74 & -3.42 & -4.41 \\
\hline 3.40 & -19.86 & 1.11 & -2.86 & -0.49 & -3.41 & -1.85 & -4.17 & -3.40 & -4.75 \\
\hline
\end{tabular}


Table D-5. (Continued).

\begin{tabular}{|c|c|c|c|c|c|c|c|c|c|}
\hline \multicolumn{10}{|c|}{ Plantarflexion } \\
\hline \multicolumn{2}{|c|}{ Passive (0\%) } & \multicolumn{2}{|c|}{$25 \%$} & \multicolumn{2}{|c|}{$50 \%$} & \multicolumn{2}{|c|}{$75 \%$} & \multicolumn{2}{|c|}{$100 \%$} \\
\hline $\mathbf{X}_{\mathrm{IAR}}$ & $Z_{\text {IAR }}$ & $\mathbf{X}_{\mathrm{IAR}}$ & $Z_{\mathrm{IAR}}$ & $X_{\text {IAR }}$ & $\mathrm{Z}_{\mathrm{IAR}}$ & $\mathbf{X}_{\text {IAR }}$ & $\mathbf{Z}_{\mathrm{IAR}}$ & $\mathbf{X}_{\text {IAR }}$ & $Z_{\text {IAR }}$ \\
\hline 3.24 & -20.95 & 1.21 & -3.44 & -0.42 & -3.57 & -1.78 & -4.63 & -3.38 & -5.11 \\
\hline 3.06 & -22.08 & 1.31 & -4.06 & -0.34 & -3.77 & -1.71 & -5.13 & -3.35 & -5.51 \\
\hline 2.89 & -23.23 & 1.41 & -4.70 & -0.26 & -3.99 & -1.64 & -5.65 & -3.33 & -5.94 \\
\hline 2.70 & -24.41 & 1.50 & -5.38 & -0.18 & -4.25 & -1.58 & -6.21 & -3.30 & -6.39 \\
\hline 2.51 & -25.62 & 1.60 & -6.09 & -0.10 & -4.53 & -1.51 & -6.80 & -3.28 & -6.88 \\
\hline 2.32 & -26.86 & 1.69 & -6.83 & -0.02 & -4.85 & -1.44 & -7.41 & -3.26 & -7.40 \\
\hline 2.12 & -28.14 & 1.78 & -7.59 & 0.07 & -5.19 & -1.38 & -8.06 & -3.23 & -7.95 \\
\hline
\end{tabular}


Table D-6. Kinematic Study IAR Values ( $\quad$ mm) for Specimen 4.

\begin{tabular}{|c|c|c|c|c|c|c|c|c|c|}
\hline \multicolumn{10}{|c|}{ Dorsiflexion } \\
\hline \multicolumn{2}{|c|}{ Passive $(0 \%)$} & \multicolumn{2}{|c|}{$25 \%$} & \multicolumn{2}{|c|}{$50 \%$} & \multicolumn{2}{|c|}{$75 \%$} & \multicolumn{2}{|c|}{$100 \%$} \\
\hline $\mathbf{X}_{\text {IAR }}$ & $\mathbf{Z}_{\text {IAR }}$ & $\mathbf{X}_{\text {IAR }}$ & $\mathbf{Z}_{\text {IAR }}$ & $\mathbf{X}_{\text {IAR }}$ & $\mathbf{Z}_{\text {IAR }}$ & $\mathbf{X}_{\text {IAR }}$ & $\mathbf{Z}_{\text {IAR }}$ & $\mathbf{X}_{\text {IAR }}$ & $\mathbf{Z}_{\text {IAR }}$ \\
\hline-0.30 & -19.68 & 0.52 & -5.38 & -1.01 & -0.12 & -0.91 & -8.58 & -2.38 & -4.71 \\
\hline-0.26 & -19.45 & 0.40 & -4.98 & -1.09 & -0.10 & -1.06 & -7.95 & -2.43 & -4.32 \\
\hline-0.22 & -19.22 & 0.26 & -4.55 & -1.18 & -0.09 & -1.22 & -7.28 & -2.49 & -3.92 \\
\hline-0.18 & -19.01 & 0.12 & -4.14 & -1.27 & -0.11 & -1.40 & -6.64 & -2.56 & -3.55 \\
\hline-0.13 & -18.84 & -0.03 & -3.77 & -1.35 & -0.16 & -1.57 & -6.03 & -2.62 & -3.21 \\
\hline-0.10 & -18.69 & -0.18 & -3.43 & -1.44 & -0.24 & -1.76 & -5.45 & -2.69 & -2.89 \\
\hline-0.06 & -18.57 & -0.33 & -3.11 & -1.53 & -0.35 & -1.95 & -4.90 & -2.77 & -2.61 \\
\hline-0.03 & -18.47 & -0.49 & -2.83 & -1.62 & -0.48 & -2.15 & -4.37 & -2.85 & -2.35 \\
\hline 0.00 & -18.41 & -0.66 & -2.57 & -1.71 & -0.65 & -2.35 & -3.88 & -2.93 & -2.12 \\
\hline 0.03 & -18.38 & -0.82 & -2.34 & -1.80 & -0.84 & -2.56 & -3.42 & -3.01 & -1.92 \\
\hline 0.06 & -18.37 & -0.99 & -2.14 & -1.90 & -1.06 & -2.77 & -2.99 & -3.10 & -1.74 \\
\hline 0.09 & -18.40 & -1.17 & -1.97 & -1.99 & -1.31 & -2.99 & -2.58 & -3.20 & -1.60 \\
\hline 0.11 & -18.45 & -1.34 & -1.84 & -2.08 & -1.58 & -3.22 & -2.21 & -3.30 & -1.48 \\
\hline 0.13 & -18.53 & -1.53 & -1.72 & -2.17 & -1.89 & -3.45 & -1.86 & -3.40 & -1.40 \\
\hline 0.15 & -18.64 & -1.71 & -1.64 & -2.26 & -2.22 & -3.69 & -1.55 & -3.50 & -1.34 \\
\hline 0.16 & -18.78 & -1.90 & -1.59 & -2.35 & -2.59 & -3.94 & -1.26 & -3.61 & -1.31 \\
\hline 0.18 & -18.94 & -2.09 & -1.57 & -2.44 & -2.98 & -4.19 & -1.01 & -3.72 & -1.30 \\
\hline 0.19 & -19.14 & -2.29 & -1.58 & -2.54 & -3.40 & -4.45 & -0.78 & -3.84 & -1.33 \\
\hline 0.20 & -19.36 & -2.49 & -1.61 & -2.63 & -3.84 & -4.71 & -0.59 & -3.96 & -1.39 \\
\hline 0.21 & -19.62 & -2.70 & -1.68 & -2.72 & -4.32 & -4.98 & -0.42 & -4.08 & -1.47 \\
\hline
\end{tabular}

\section{Plantarflexion}

\begin{tabular}{|c|c|c|c|c|c|c|c|c|c|}
\hline \multicolumn{2}{|c|}{ Passive (0\%) } & \multicolumn{2}{|c|}{$25 \%$} & \multicolumn{2}{|c|}{$50 \%$} & \multicolumn{2}{|c|}{$75 \%$} & \multicolumn{2}{|c|}{$100 \%$} \\
\hline $\mathbf{X}_{\text {IAR }}$ & $\mathbf{Z}_{\text {IAR }}$ & $\mathbf{X}_{\text {IAR }}$ & $\mathbf{Z}_{\text {IAR }}$ & $\mathbf{X}_{\text {IAR }}$ & $\mathbf{Z}_{\text {IAR }}$ & $\mathbf{X}_{\text {IAR }}$ & $\mathbf{Z}_{\text {IAR }}$ & $\mathbf{X}_{\text {IAR }}$ & $\mathbf{Z}_{\text {IAR }}$ \\
\hline 1.83 & -19.52 & 2.95 & 3.09 & 1.42 & -3.19 & 1.82 & -0.32 & -0.45 & -3.63 \\
\hline 1.80 & -19.37 & 3.09 & 2.80 & 1.53 & -3.11 & 1.91 & -0.24 & -0.38 & -3.38 \\
\hline 1.77 & -19.23 & 3.25 & 2.45 & 1.65 & -3.06 & 2.02 & -0.19 & -0.30 & -3.12 \\
\hline 1.74 & -19.13 & 3.40 & 2.08 & 1.77 & -3.03 & 2.13 & -0.16 & -0.22 & -2.90 \\
\hline 1.71 & -19.05 & 3.55 & 1.67 & 1.89 & -3.03 & 2.24 & -0.16 & -0.13 & -2.70 \\
\hline 1.69 & -19.00 & 3.70 & 1.24 & 2.02 & -3.06 & 2.35 & -0.18 & -0.04 & -2.53 \\
\hline 1.67 & -18.97 & 3.85 & 0.78 & 2.15 & -3.12 & 2.46 & -0.24 & 0.05 & -2.39 \\
\hline 1.65 & -18.98 & 3.99 & 0.29 & 2.28 & -3.21 & 2.57 & -0.32 & 0.14 & -2.28 \\
\hline 1.63 & -19.02 & 4.13 & -0.23 & 2.41 & -3.33 & 2.69 & -0.44 & 0.24 & -2.19 \\
\hline 1.61 & -19.08 & 4.27 & -0.78 & 2.54 & -3.48 & 2.80 & -0.58 & 0.34 & -2.14 \\
\hline 1.60 & -19.18 & 4.41 & -1.35 & 2.67 & -3.65 & 2.92 & -0.75 & 0.45 & -2.11 \\
\hline 1.58 & -19.30 & 4.55 & -1.96 & 2.80 & -3.85 & 3.04 & -0.95 & 0.55 & -2.11 \\
\hline 1.57 & -19.45 & 4.68 & -2.59 & 2.94 & -4.09 & 3.16 & -1.18 & 0.66 & -2.14 \\
\hline
\end{tabular}


Table D-6. (Continued).

\begin{tabular}{|c|c|c|c|c|c|c|c|c|c|}
\hline \multicolumn{10}{|c|}{ Plantarflexion } \\
\hline \multicolumn{2}{|c|}{ Passive (0\%) } & \multicolumn{2}{|c|}{$25 \%$} & \multicolumn{2}{|c|}{$50 \%$} & \multicolumn{2}{|c|}{$75 \%$} & \multicolumn{2}{|c|}{$100 \%$} \\
\hline $\mathbf{X}_{\mathrm{IAR}}$ & $Z_{\text {IAR }}$ & $\mathbf{X}_{\mathrm{IAR}}$ & $Z_{\mathrm{IAR}}$ & $\mathbf{X}_{\mathrm{IAR}}$ & $\mathrm{Z}_{\mathrm{IAR}}$ & $\mathbf{X}_{\mathrm{IAR}}$ & $\mathbf{Z}_{\mathrm{IAR}}$ & $\mathbf{X}_{\mathrm{IAR}}$ & $Z_{\text {IAR }}$ \\
\hline 1.57 & -19.63 & 4.81 & -3.26 & 3.08 & -4.35 & 3.29 & -1.43 & 0.77 & -2.20 \\
\hline 1.56 & -19.84 & 4.94 & -3.95 & 3.21 & -4.64 & 3.41 & -1.72 & 0.89 & -2.29 \\
\hline 1.56 & -20.08 & 5.07 & -4.67 & 3.35 & -4.96 & 3.53 & -2.03 & 1.01 & -2.40 \\
\hline 1.56 & -20.34 & 5.20 & -5.42 & 3.50 & -5.31 & 3.66 & -2.37 & 1.13 & -2.55 \\
\hline 1.56 & -20.64 & 5.32 & -6.20 & 3.64 & -5.69 & 3.79 & -2.74 & 1.25 & -2.72 \\
\hline 1.56 & -20.96 & 5.45 & -7.00 & 3.78 & -6.09 & 3.92 & -3.14 & 1.38 & -2.92 \\
\hline 1.57 & -21.31 & 5.57 & -7.84 & 3.93 & -6.53 & 4.05 & -3.57 & 1.51 & -3.15 \\
\hline
\end{tabular}




\section{VITA}

Kelly Nadine Salb was born in Bridgeport, Connecticut in 1987. She was raised in Fairfield, Connecticut where she graduated from Fairfield Warde High School in 2005. She attended Allegheny College and graduated with a Bachelor of Science in Physics in 2009. After taking a year off to travel the United States, she returned to school to earn a Master of Science degree in Biomedical Engineering at the University of Tennessee Health Science Center. She entered as a student of Dr. Kaushik Parthasarathi to study chemical injury pathways in the rat lung using fluorescent microscopy. In May 2011, she joined Dr. Denis DiAngelo's Biorobotics Laboratory, focusing on the biomechanical effects of bone loading protocols in mice and then moved to studying biomechanical studies of the foot and ankle complex in February, 2012. 